\title{
Search for a Fermionic Top Partner in Unexplored Decay Modes
}

by

\author{
Hassan Easa
}

\begin{abstract}
A thesis submitted to the Faculty of Graduate and Postdoctoral Affairs in partial fulfillment of the requirements for the degree of
\end{abstract}

\author{
Master of Science \\ in \\ Physics
}
Ottawa-Carleton Institute for Physics
Carleton University
Ottawa, Ontario

17 July, 2017

(C) 2017

Hassan Easa 
The undersigned hereby recommends to the

Faculty of Graduate and Postdoctoral Affairs acceptance of the thesis

\title{
Search for a Fermionic Top Partner in Unexplored Decay Modes
}

\author{
submitted by Hassan Easa \\ in partial fulfillment of the requirements for the degree of \\ Master of Science \\ in \\ Physics
}

Professor Thomas Gregoire, Thesis Co-supervisor

Professor Daniel Stolarski, Thesis Co-supervisor

Professor Alain Bellerive, Chair, Department of Physics

Ottawa-Carleton Institute for Physics

Department of Physics

Carleton University

August, 2017 


\section{Abstract}

We investigate the existence of a new fermionic partner to the top quark in light of the current experimental searches conducted at the colliders. The model presented here is phenomenologically attractive because it is less constrained than the traditional decay modes of the top partner. It also has the potential to cancel the quadratic divergences in the Higgs mass. In addition to the new fermion, the model contains another scalar particle that mediates interactions with the Standard Model light quarks. This new fermion is studied by using various recasted searches for previously unexplored decay $\operatorname{channels}(T \longrightarrow c \eta \quad, T \longrightarrow t \eta \quad$ where $\eta \longrightarrow \bar{u} u)$. We thoroughly study the phenomenology of such model. From experimental searches, we find that current LHC searches exclude the top partner mass (new fermion) of about $350 \mathrm{GeV}$ (and some other points in the $m_{T} m_{\eta}$-plane) for its decay to light quarks. On the other hand, when the top partner decays to a top and two light quarks, the lower limit obtained on the top partner mass can be as large as $650 \mathrm{GeV}$. 


\section{Acknowledgments}

I would like begin by expressing my profound gratitude to my co-supervisor Dr. Thomas Gregoire for his excellent guidance and advice throughout my research. Also, I would like to thank my co-supervisor Dr. Daniel Stolarski for his participation in my research and his endless enthusiasm to carry this research forward. I am very grateful to my supervisors for their guidance and their prompt response to my queries. Furthermore, I would like to thank Kevin Earl for his help with the technical aspects of the analysis. Outside the academic circle, I am very grateful for the immense support of my family throughout my academic endeavours. 


\section{Statement of Originality}

To the best of my knowledge, the content of this thesis is my own work and all the assistance received in preparing this thesis and the sources used have been properly acknowledged. 


\section{Table of Contents}

Abstract $\quad$ iii

Acknowledgments $\quad$ iv

Statement of Originality $\quad$ v

Table of Contents $\quad$ vi

List of Tables $\quad$ ix

List of Figures $\quad$ x

1 Introduction 1

2 Standard Model $\quad 6$

2.1 Notation and Units . . . . . . . . . . . . . . . . 6

2.2 Basic Lagrangian Mechanics . . . . . . . . . . . . . . . . . 9

2.2.1 Principle of Least Action . . . . . . . . . . . . . . . . . . . 10

2.3 Gauge Invariance . . . . . . . . . . . . . . . . . . . . . 12

2.4 Spontaneous Symmetry Breaking . . . . . . . . . . . . . . . 14

2.5 Gauge Boson Masses . . . . . . . . . . . . . . . . . . . . . . . . . . . 19

2.6 Lepton Masses . . . . . . . . . . . . . . . . . . . . . . . . . 24

2.7 Quark Masses ... . . . . . . . . . . . . . . 28 
2.8 Kinetic Terms . . . . . . . . . . . . . . . . . . . . . 31

2.9 Overview of QCD . . . . . . . . . . . . . . . 35

3 Our Model and Motivation $\quad 37$

3.1 Motivation . . . . . . . . . . . . . . . 37

3.2 Our Model . . . . . . . . . . . . . . . . . . 40

3.3 Our Model Phenomenology . . . . . . . . . . . . . . 46

3.3.1 Case 1: Top Partner Decay to Light Quarks . . . . . . . . . . 47

3.3.2 Case 2: Top Partner Decay to a Heavy Flavour Quark . . . . 48

4 Analysis and Strategies $\quad 50$

4.1 Implementation Tools . . . . . . . . . . . . . . . . . . . . 52

4.2 QCD Multi-jet Background . . . . . . . . . . . . . . 54

4.3 Matching and Merging . . . . . . . . . . . . . . 55

4.4 Pythia6 VS Pythia8 . . . . . . . . . . . . . . . . 58

5 Results and Discussion $\quad 62$

5.1 Case I: Light Flavour Results . . . . . . . . . . . . . . . . . . . . 62

5.1 .1 CMS Search . . . . . . . . . . . . . . . 62

5.1 .2 ATLAS Search . . . . . . . . . . . . 67

5.2 Case II: Heavy Flavour Results . . . . . . . . . . . . . . . . . . . . . 74

5.2.1 CMS Leptonic Search . . . . . . . . . . . . . . 75

5.2.2 CMS Hadronic Search . . . . . . . . . . . . . . . 80

5.2.3 ATLAS Hadronic Search . . . . . . . . . . . . . . . . 82

6 Conclusion $\quad 84$

$\begin{array}{lr}\text { A Mathematical Tools } & 86\end{array}$ 
A.1 $\mathbb{Z}_{2}$ Symmetry ..................... 86

A.2 Standard Model Groups . . . . . . . . . . . . . . . . 87

A.3 Rotation group . . . . . . . . . . . . . . . . . . . . . . . . . . 90

A.4 Lorentz Invariance . . . . . . . . . . . . . . . . . . . . . 95

A.5 Basic Statistics . . . . . . . . . . . . . . . . . . 99

B Physics Tools 103

B.1 Solution to the Dirac equation . . . . . . . . . . . . 103

B.1.1 Spin Sums . . . . . . . . . . . . . . . . 105

B.2 Traces . . . . . . . . . . . . . . . . . 106

B.3 CKM Matrix . . . . . . . . . . . . . . . 110

B.4 Colliders Coordinate System . . . . . . . . . . . . . . 110

B.5 cross section . . . . . . . . . . . . . . . . . . . . . 113

B.6 Decay Rate . . . . . . . . . . . . . . . . . . . . 116

B.7 Scalar $\eta$ Width . . . . . . . . . . . . . . . . . . . . . . . 117

B.8 Noether's Theorem . . . . . . . . . . . . . . . . . . . . 119

$\begin{array}{ll}\text { C Additional Plots } & 121\end{array}$

C.1 Pythia8 and Pythia6 . . . . . . . . . . . . . . . 121

$\begin{array}{ll}\text { List of References } & 122\end{array}$ 


\section{List of Tables}

2.1 Natural Unit conversion . . . . . . . . . . . . . . . . . . . 7

5.1 Selection requirements for $8 \mathrm{TeV}$ CMS search . . . . . . . . . . 64

5.2 ATLAS $8 \mathrm{TeV}$ search cuts . . . . . . . . . . . . 70

5.3 Selection requirements for $13 \mathrm{TeV}$ CMS search . . . . . . . . 73

5.4 Various selection criteria for the leptonic CMS analysis $\ldots \ldots \ldots 77$ 


\section{List of Figures}

1.1 One loop correction Feynman diagrams for the Higgs mass. . . . . . . 4

1.2 Standard model of particle physics . . . . . . . . . . . . 5

2.1 The Higgs potential as a function of the field for $\mu^{2}<0$. . . . . . 15

2.2 The Higgs potential as a function of the field for $\mu^{2}>0 . \ldots 16$

2.3 Flavour changing interaction Feynman diagram . . . . . . . . . . 35

3.1 The typical top partner decay channels investigated at the LHC. . . . 40

3.2 Feynman diagram for the top partner decay to light quarks. . . . . . 47

3.3 Feynman diagram for the top partner decay to a heavy and two light quarks. . . . . . . . . . . . . . . . . . . . . . 48

4.1 A schematic diagram of a typical RPV multi-jet topology . . . . . . . 51

4.2 Schematic diagram for the event generation of our desired model . . . 53

4.3 Multi-jet cross section at LHC . . . . . . . . . . . . . . 55

4.4 Top partner production cross section . . . . . . . . . . . 56

4.5 Multi-jet cross section at LHC . . . . . . . . . . . . . 58

4.6 ATLAS 8 TeV Pythia6 vs Pythia8 limits . . . . . . . . . . . . 59

4.7 ATLAS 8 TeV Pythia6 vs Pythia8 with one Master Switch on . . . . 60

4.8 ATLAS 8 TeV Pythia6 vs Pythia8 with only one Switch off . . . . . . 61

5.1 The plot shows the rescaled cross section at $\sqrt{s}=8 \mathrm{TeV}$ as a function of the top partner mass for various scalar masses. . . . . . . . . . . 66 
5.2 Light flavour exclusion curve . . . . . . . . . . . . . . 67

5.3 CMS 7TeV hadronic search cross section for various $m_{\eta} \ldots \ldots 8$

5.4 ATLAS $8 \mathrm{TeV}$ upper limit curve for the light flavour top partner . . . 71

5.5 ATLAS $13 \mathrm{TeV}$ 4J visible cross section limit . . . . . . . . . 73

5.6 ATLAS $13 \mathrm{TeV}$ 5J visible cross section limit . . . . . . . . . . 74

5.7 ATLAS 8TeV Heavy flavour limit . . . . . . . . . . . . . 76

5.8 CMS 8TeV leptonic search requiring an electron . . . . . . . . . . . 77

5.9 CMS 8TeV leptonic search requiring a muon . . . . . . . . . . . 78

5.10 The figure shows the exclusion curve using the lepton analysis for the top partner decay to a top quark and two light SM quarks. . . . . . . 79

5.11 CMS $8 \mathrm{TeV}$ Heavy flavour limit . . . . . . . . . . . . . . . 80

5.12 The figure shows the exclusion curve from the fully hadronic CMS analysis for the top partner decay to a heavy and two light jets. . . . 81

5.13 ATLAS 8TeV Heavy flavour limit . . . . . . . . . . . . . . 82

A.1 A simple rotation in the xy-plane by an angle $\theta$ counterclockwise. . . 91

B.1 A typical coordinate system used in colliders. . . . . . . . . . . . . . 110

C.1 CMS 8TeV Pythia6 vs Pythia8 limits . . . . . . . . . . . . 121 


\section{Chapter 1}

\section{Introduction}

There are four known fundamental forces in nature, which describe everything around us. Firstly, the gravitational force acts between any massive objects and is always attractive. The gravitational force, from a quantum field theory perspective is mediated by a hypothetical particle called the graviton. It is the most familiar of all the forces. However, it is the least understood of all as we don't have a complete picture using field theory. Secondly, the electromagnetic force is generated by any charged object and is mediated by the photon, a quanta of light. The electromagnetic force can be attractive or repulsive depending on the charges of the objects involved. Beside its immense importance in daily applications, electromagnetism led to the development of relativity and modern physics. Thirdly, the weak nuclear force is responsible for many radioactive decays and it is mediated by the $\mathrm{W}$ or $\mathrm{Z}$ bosons, also called gauge bosons. It is responsible for keeping the sun burning and ultimately life on earth. Finally, the strong nuclear force is responsible for holding neutrons and protons to-

gether and it is mediated by massless particles called gluons. The strong nuclear force is responsible for holding all the ordinary matter together since it binds quarks to form hadrons(subatomic particles made up of quarks). 
All the forces except gravity, are nicely incorporated into a mathematical framework called the Standard Model (SM) and all the particles of the SM are shown in figure 1.2. The Standard Model explains how the fundamental particles behave and interact with each other. Over the last few decades, the SM has been tested extensively and so far it is has been in excellent agreement with experiments. With the discovery of the Higgs boson, the SM is complete in all its glory. However, it still leaves many questions unanswered. For example, one expects the big bang should have produced equal amount of matter and antimatter. Because the early universe was really hot and matter and anti-matter particles are always produced in pairs, they would annihilate each other upon contact leaving only energy behind. However from observation, there is an abundance of baryonic matter in the universe. This is known as the baryon asymmetry and the SM doesn't give any explanation. Furthermore, from cosmological observations we know that the SM explains only $5 \%$ of the energy content of our universe. There is a strong observational evidence which suggests that nearly $30 \%$ of it is a new kind of matter called dark matter [1]. Dark matter only interacts through gravity with ordinary matter and it doesn't emit electromagnetic radiation. It may couple to the SM particles but very weakly. All the evidence suggest that dark matter is composed of a new type of particle but the SM doesn't contain a possible candidate. Also, the SM doesn't include gravity at all. So it is obvious that our current theoretical picture of nature is incomplete. Thus from an experimental and theoretical point of view, it is extremely desirable to search for physics beyond the SM. Furthermore, quantum field theory suggests that the Higgs vacuum expectation value (the Higgs field value at the vacuum) should get contributions from all the energy scales ( i.e. Planck scale $\approx 10^{19} \mathrm{GeV}$ ) but it doesn't. The Higgs vacuum expectation value $(v \approx 246 \mathrm{GeV})$, which determines the masses of the gauge bosons ( $\mathrm{W}$ and $\mathrm{Z}$ ), is much smaller than the Planck scale. So the question is, 
why is there such a hierarchy between the two scales? This is essentially the hierarchy problem and the SM doesn't provide any solution but instead one must rely on some miraculous cancellation between the parameters.

There are many viable candidates for physics beyond the SM, but there has not been any evidence in favour of one over another. For example, the most prominent theory that extends the SM is Supersymmetry (SUSY), which in its minimal extension double the number of particles [2]. SUSY is a spacetime symmetry between fermions (half integer spin particles) and bosons (integer spin particles). So SUSY associates a fermion with its super-partner which is a boson and vice versa. The interesting aspect of SUSY is that it can contain a natural dark matter (DM) candidate, which is the lightest supersymmetric partner (LSP). Imposing a symmetry called R-parity, a $\mathbb{Z}_{2}$ symmetry under which SM particles are even and superpartners are odd, on the model leads to a stable LSP and it implies that SUSY particles are produced in pairs. In addition, SUSY provides a solution to the hierarchy problem.

The Higgs mechanism is an essential part of the SM since it is responsible for generating masses for many of the observed particles. In fact explicit mass terms for fermions and the gauge bosons are forbidden by the structure of the SM symmetry (refer to Chapter 2 for more details). The masses of the gauge bosons and fermions are obtained when the Higgs field takes a non-zero vacuum expectation value (vev) which leads to spontaneous symmetry breaking(a processes of symmetry breaking where the Lagrangian respect the symmetry but the vacuum doesn't). The excitation of the Higgs field gives rise to the well known Higgs boson, which was a long sought after missing piece of the SM until its discovery by the Large Hadron Collider (LHC) at CERN on 04-July-2012 [3,4]. Experimental observations of the Higgs boson have established its mass to be $\mathrm{m}_{H} \approx 125 \mathrm{GeV}$. Unlike the gauge bosons or fermions of the SM, the Higgs boson is a scalar so its mass is not protected by symmetry. The Higgs 


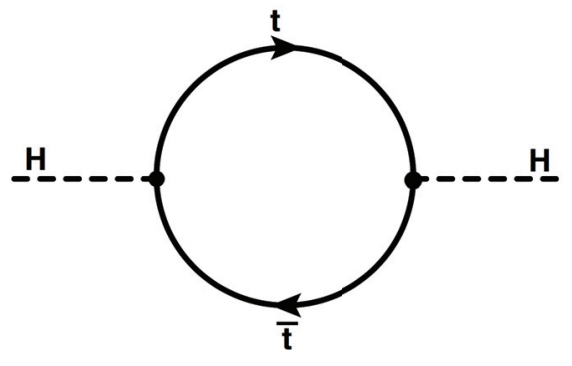

(a) Top quark loop

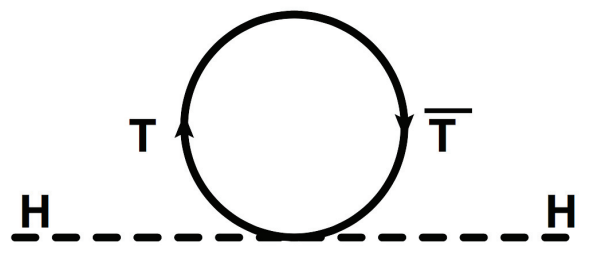

(b) Top partner Loop

Figure 1.1: One loop correction Feynman diagrams for the Higgs mass.

couple most strongly to the top quark as it is the heaviest particle. As such the one loop correction to the Higgs mass as shown by the Feynman diagram in figure 1.1 is in fact quadratically divergent:

$$
\mu_{\text {phys }}^{2} \simeq \mu^{2}-\frac{y_{t}^{2}}{16 \pi^{2}} \Lambda^{2}
$$

where $y_{t}$ is the Yukawa coupling of the top quark, $\Lambda$ is the scale at which the new physics will appear (Ultraviolet cut off), and $\mu$ is a parameter in the quadratic term of the Higgs potential (equation 2.28). In equation 1.1, $\mu$ cannot be much greater than an order of $\approx 10^{2} \mathrm{GeV}$; otherwise fine tuning is needed. So one can see that unless there is extreme fine-tuning, the Higgs mass can take on a very large unphysical value. Thus, there is the need for a model to cancel this in a natural way.

With all the motivations stated above and the many shortcomings of the SM, we have to reach out and investigate plausible alternatives to discovering new physics. Many such alternatives include a top partner responsible for cancelling quadratic divergence and solving the hierarchy problem. We study a model that introduces such a top partner that behaves the same way as the SM quarks under the strong force and has an elementary charge of $+\frac{2}{3}$. In fact such top partner has been studied at colliders $[5,6]$ but we focus on different decay channels that have not been studied 
so far. We will discuss the intricate collider phenomenology and present the exclusion plots for our proposed decay channels.

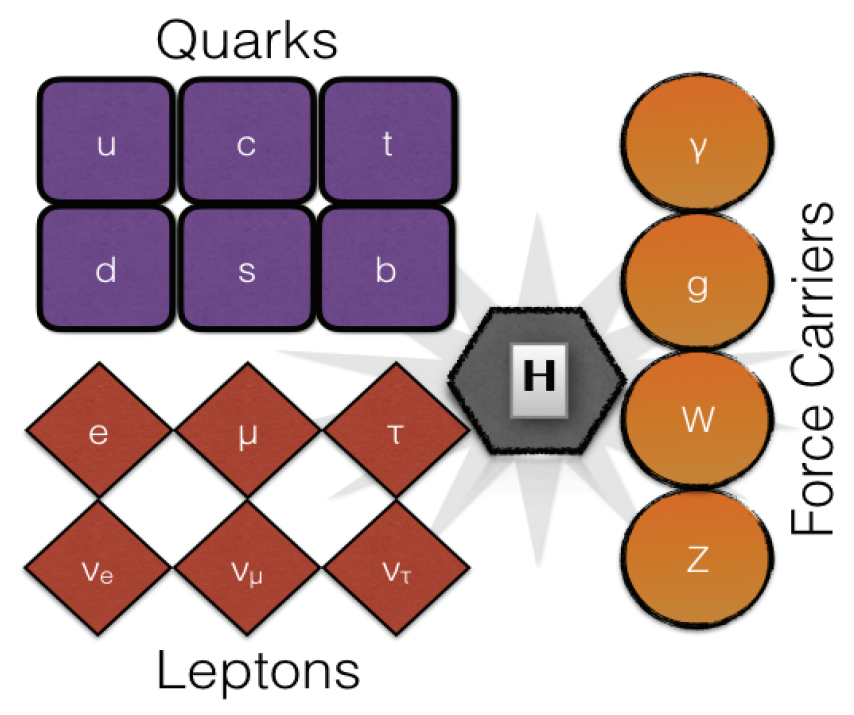

Figure 1.2: All the particle content of the Standard model of physics.

The thesis is organized into the following chapters. Firstly, Chapter 2 describes the basic notations and a theoretical overview of some of the fundamental concepts of the SM. Then, in Chapter 3 we introduce our model and describe its collider phenomenology. With the introduction of the model, we discuss the main feature including coupling to the SM quarks. Furthermore, Chapter 4 is dedicated to specific tools utilized in implementing the model and we focus on the relevant LHC searches. In Chapter 5 we present the constraints on the mass of the top partners that can be established by recasting various LHC searches. We find relatively weak bounds when the top partner decays to light jets only and a bound of roughly $650 \mathrm{GeV}$ when the top partner decays to top quark. 


\section{Chapter 2}

\section{Standard Model}

\subsection{Notation and Units}

Lets begin with some notation and unit conventions that will be used throughout the text. Firstly, we use the most common particle physics units system known as natural units, where:

$$
\hbar=c=1
$$

Then, accordingly one obtains:

$$
[\text { mass }]=[\text { Energy }]=[\text { time }]^{-1}=[\text { length }]^{-1}
$$

Furthermore, conversion between natural units and the International System of units (SI) for some quantities are shown in table 2.1. 
The metric tensor used is:

$$
g_{\mu \nu}=g^{\mu \nu}=\left[\begin{array}{cccc}
1 & 0 & 0 & 0 \\
0 & -1 & 0 & 0 \\
0 & 0 & -1 & 0 \\
0 & 0 & 0 & -1
\end{array}\right]
$$

The Greek indices always run over 0,1,2,3 (or t, $\mathrm{x}, \mathrm{y}, \mathrm{z}$ ) and the Roman indices run over 1,2,3. Since Quantum Field Theory (QFT) combines special relativity and quantum mechanics, we use four-vectors which are denoted as:

$$
x_{\mu}=\left(x_{0},-\boldsymbol{x}\right) \quad x^{\mu}=g^{\mu \nu} x_{\nu}=\left(x^{0}, \boldsymbol{x}\right)
$$

In addition, the derivative operator is:

$$
\partial_{\mu} \equiv \frac{\partial}{\partial x^{\mu}}=\left(\frac{\partial}{\partial x^{0}}, \nabla\right)
$$

We are often dealing with symmetries which are described by mathematical groups

Table 2.1: Conversion between the natural and SI units for various quantities that are used in particle physics.

\begin{tabular}{|c|c|c|c|}
\hline Quantity & Natural Units & SI units & Converted Values \\
\hline Energy & $\mathrm{E}$ & $\mathrm{kg} \cdot \frac{m^{2}}{s^{2}}$ & $1 \mathrm{GeV}=1.602 \times 10^{-10} \mathrm{~J}$ \\
Momentum & $\mathrm{E}$ & $\mathrm{kg} \cdot \frac{m}{\mathrm{~s}}$ & $(1 \mathrm{GeV})^{-1}=5.34 \times 10^{-19} \mathrm{~kg} \cdot \frac{m}{\mathrm{~s}}$ \\
Time & $\frac{1}{E}$ & $\mathrm{~s}$ & $(1 \mathrm{GeV})^{-1}=6.583 \times 10^{-25} \mathrm{~s}$ \\
Length & $\frac{1}{E}$ & $\mathrm{~m}$ & $(1 \mathrm{GeV})^{-1}=1.975 \times 10^{-16} \mathrm{~m}$ \\
Cross Section & $\frac{1}{E^{2}}$ & $m^{2}$ & $(1 \mathrm{GeV})^{-2}=3.9 \times 10^{-32} \mathrm{~m}^{2}$ \\
\hline
\end{tabular}


and the well known Pauli sigma matrices form the basis for one such group. The Pauli matrices are:

$$
\sigma^{1}=\left[\begin{array}{ll}
0 & 1 \\
1 & 0
\end{array}\right] \quad \sigma^{2}=\left[\begin{array}{cc}
0 & -i \\
i & 0
\end{array}\right] \quad \sigma^{3}=\left[\begin{array}{cc}
1 & 0 \\
0 & -1
\end{array}\right]
$$

and they satisfies the following commutation relations

$$
\left[\sigma^{a}, \sigma^{b}\right]=\sigma^{a} \sigma^{b}-\sigma^{b} \sigma^{a}=2 i \epsilon^{a b c} \sigma^{c}
$$

where the antisymmetric tensor is:

$$
\epsilon^{123}=+1
$$

Furthermore, in Quantum field theory, one often uses the gamma matrices often called the Dirac matrices. These $4 \times 4$ matrices satisfy the following anti-commutation relation:

$$
\left\{\gamma^{\mu}, \gamma^{\nu}\right\} \equiv \gamma^{\mu} \gamma^{\nu}+\gamma^{\nu} \gamma^{\mu}=2 g^{\mu \nu} \mathbb{I}_{4 \times 4}
$$

Explicitly, the gamma matrices in the Weyl or Chiral representation in 2 by 2 block form are:

$$
\gamma^{0}=\left(\begin{array}{ll}
0 & 1 \\
1 & 0
\end{array}\right), \quad \gamma^{j}=\left(\begin{array}{cc}
0 & \sigma^{j} \\
-\sigma^{j} & 0
\end{array}\right)
$$


Also, one can define a new matrix with the following properties:

$$
\begin{gathered}
\gamma^{5} \equiv i \gamma^{0} \gamma^{1} \gamma^{2} \gamma^{3}, \quad \gamma^{5}=\left(\begin{array}{cc}
-1 & 0 \\
0 & 1
\end{array}\right) \\
\left(\gamma^{5}\right)^{\dagger}, \quad\left(\gamma^{5}\right)^{2}=1, \quad\left\{\gamma^{5}, \gamma^{\mu}\right\}=0
\end{gathered}
$$

\subsection{Basic Lagrangian Mechanics}

In classical mechanics, the Lagrangian is defined as

$$
L=T(\text { Kinetic Energy })-V(\text { Potential Energy })
$$

The Lagrangian $L(q, \dot{q})$ depends on the generalized coordinates $q$ and their time derivative. Generalized coordinates are a set of parameters that describe the configuration of a dynamical system with respect to some reference configuration. For a particular system, a specific coordinate system (cartesian, spherical, etc) is chosen based on the symmetry of the system. In Quantum Field Theory (QFT), the generalized coordinates are replaced by quantum fields

$$
\begin{aligned}
& q \longrightarrow \phi(x) \\
& \dot{q} \longrightarrow \partial_{\mu} \phi(x)
\end{aligned}
$$

A quantum field $\phi(x)$ is a quantity that has a value at every point in space and time. Then the dynamics of the fields are governed by the Lagrangian and one of the benefits of the Lagrangian is that its invariance (under a symmetry transformation) 
leads to conserved quantities (Appendix B.8).

$$
L=\int \mathcal{L}\left(\phi(x), \partial_{\mu} \phi(x)\right) d^{3} x
$$

where $\mathcal{L}$ is the Lagrangian density(this is most commonly called just the Lagrangian). Now in classical mechanics, one of the fundamental quantity of interest is the action, $S$, which is given as:

$$
S=\int L d t=\int \mathcal{L}\left(\phi(x), \partial_{\mu} \phi(x)\right) d^{4} x
$$

Besides the Lagrangian description of a system, in classical mechanics one can use the Hamiltonian to represent a dynamical system. The Hamiltonian is defined in terms of the Lagrangian as follow:

$$
H \equiv \sum p \dot{q}-L, \quad \text { where } \dot{q}=\frac{\partial q}{\partial t}, p=\frac{\partial L}{\partial \dot{q}}
$$

As usual going into the continuous case and replacing generalized coordinates with fields, one arrives at:

$$
H=\int d^{3} x[\pi(\mathbf{x}) \dot{\phi}(\mathbf{x})-\mathcal{L}] \equiv \int d^{3} x \mathcal{H}, \quad \text { where } \pi(\mathbf{x}) \equiv \frac{\partial \mathcal{L}}{\partial \dot{\phi}(\mathbf{x})}
$$

\subsubsection{Principle of Least Action}

In order to obtain the equation of motion, an extremum of the action $S$ has to be found. The principle of least action states that the evolution of the system between time $t_{1}$ and $t_{2}$ is such that $S$ is an extremum [7]. Under variation, the field and its 
derivative becomes

$$
\begin{gathered}
\phi \longrightarrow \phi^{\prime}=\phi+\delta \phi \\
\partial_{\mu} \phi \longrightarrow \partial_{\mu} \phi^{\prime}=\partial_{\mu} \phi+\delta\left(\partial_{\mu} \phi\right)
\end{gathered}
$$

Consequently, one obtains

$$
\delta\left(\partial_{\mu} \phi\right)=\partial_{\mu} \phi^{\prime}-\partial_{\mu} \phi=\partial_{\mu}\left(\phi^{\prime}-\phi\right)=\partial_{\mu}(\delta \phi)
$$

So applying the variational principle to the action,

$$
\begin{aligned}
0 & =\delta S \\
& =\int\left[\frac{\partial \mathcal{L}}{\partial \phi} \delta \phi+\frac{\partial \mathcal{L}}{\partial\left(\partial_{\mu} \phi\right)} \delta\left(\partial_{\mu} \phi\right)\right] d^{4} x \\
& =\int\left[\frac{\partial \mathcal{L}}{\partial \phi} \delta \phi+\frac{\partial \mathcal{L}}{\partial\left(\partial_{\mu} \phi\right)} \partial_{\mu}(\delta \phi)-\partial_{\mu}\left(\frac{\partial \mathcal{L}}{\partial\left(\partial_{\mu} \phi\right)}\right) \delta \phi+\partial_{\mu}\left(\frac{\partial \mathcal{L}}{\partial\left(\partial_{\mu} \phi\right)}\right) \delta \phi\right] d^{4} x \\
& =\int \delta \phi\left[\frac{\partial \mathcal{L}}{\partial \phi}-\partial_{\mu}\left(\frac{\partial \mathcal{L}}{\partial\left(\partial_{\mu} \phi\right)}\right)\right] d^{4} x+\int\left[\frac{\partial \mathcal{L}}{\partial\left(\partial_{\mu} \phi\right)} \partial_{\mu}(\delta \phi)+\partial_{\mu}\left(\frac{\partial \mathcal{L}}{\partial\left(\partial_{\mu} \phi\right)}\right) \delta \phi\right] d^{4} x \\
& =\int \delta \phi\left[\frac{\partial \mathcal{L}}{\partial \phi}-\partial_{\mu}\left(\frac{\partial \mathcal{L}}{\partial\left(\partial_{\mu} \phi\right)}\right)\right] d^{4} x+\int \partial_{\mu}\left(\frac{\partial \mathcal{L}}{\partial\left(\partial_{\mu} \phi\right)} \delta \phi\right) d^{4} x
\end{aligned}
$$

Now the last term of the integration is zero because by Gauss's divergence theorem, it can be converted into a surface integral and $\delta \phi$ is assumed to be zero on the surface. Thus we get

$$
0=\int \delta \phi\left[\frac{\partial \mathcal{L}}{\partial \phi}-\partial_{\mu}\left(\frac{\partial \mathcal{L}}{\partial\left(\partial_{\mu} \phi\right)}\right)\right] d^{4} x
$$


Because the whole integral must be zero for an arbitrary $\delta \phi$, then the term multiplying $\delta \phi$ must vanish for all points. Thus we get the Euler - Lagrange equation of motion

$$
0=\frac{\partial \mathcal{L}}{\partial \phi}-\partial_{\mu}\left(\frac{\partial \mathcal{L}}{\partial\left(\partial_{\mu} \phi\right)}\right)
$$

\subsection{Gauge Invariance}

We are most often interested in one particular type of field theory called a gauge theory. In a gauge theory, the Lagrangian is invariant under a set of continuous local symmetry transformations. The set of gauge transformations form a Lie group. This Lie group has its corresponding Lie algebra. We will learn that in order to ensure that the Lagrangian is gauge invariant, for each group generator we must introduce a gauge field. Exact gauge invariance implies that gauge bosons are massless. To see this, lets consider the transformation of a free scalar field:

$$
\phi \longrightarrow \phi^{\prime}=U \phi, \text { where } U=e^{i \theta}
$$

where $\theta$ is a real transformation parameter. Then it is easy to observe that the Lagrangian would be invariant under such global phase transformation.

$$
\mathcal{L}=\partial_{\mu} \phi^{*} \partial^{\mu} \phi-m^{2} \phi^{*} \phi
$$

However, making this particular transformation local $(\theta=\theta(x))$, then the Lagrangian is no longer invariant under such transformation. The mass term is still invariant, but extra terms appear due to the the derivative in the kinetic term.

$$
\phi \Longrightarrow \phi^{\prime}=e^{i \theta(x)} \phi
$$




$$
\begin{aligned}
\partial_{\mu} \phi \longrightarrow \partial_{\mu} \phi^{\prime} & =e^{i \theta(x)}\left(\partial_{\mu} \phi+i \partial_{\mu} \theta(x) \phi\right) \\
& \neq U \partial_{\mu} \phi
\end{aligned}
$$

Thus the regular derivative of the field doesn't have the same transformation at every spacetime point. In order to preserve invariance under such local transformations, one introduces a covariant derivative, which compensates for the additional terms introduced from the local phase transformation of the field. The covariant derivative is defined as:

$$
\partial_{\mu} \longrightarrow D_{\mu} \equiv \partial_{\mu}-i B_{\mu}
$$

where $B_{\mu}$ is a new field known as gauge field, also called the connection, that has the following transformation:

$$
B_{\mu} \longrightarrow B_{\mu}^{\prime}=B_{\mu}+\partial_{\mu} \theta(x)
$$

To check that this transformation leaves the Lagrangian invariant under local phase transformation, lets observe the transformation of the covariant derivative term

$$
\begin{aligned}
D_{\mu} \phi \longrightarrow\left(D_{\mu} \phi\right)^{\prime} & =\left[\partial_{\mu}-i\left(B_{\mu}+\partial_{\mu} \theta(x)\right)\right] e^{i \theta(x)} \phi \\
& =e^{i \theta(x)}\left[\partial_{\mu}-i B_{\mu}\right] \phi=e^{i \theta(x)} D_{\mu} \phi
\end{aligned}
$$

Thus the covariant derivative transforms just like the field and it makes the kinetic term invariant under local U(1) transformation. So, we have gauged the group U(1) in the theory. However, gauge invariance leads to massless gauge bosons. For example, 
a mass term of the form $\frac{1}{2} m_{\gamma} B_{\mu} B^{\mu}$ is forbidden by $\mathrm{U}(1)$ gauge transformation.

$$
\begin{aligned}
\frac{1}{2} m_{\gamma} B_{\mu} B^{\mu} \longrightarrow \frac{1}{2} m_{\gamma}\left(B_{\mu} B^{\mu}\right)^{\prime} & =\frac{1}{2} m_{\gamma}\left(B_{\mu}+\partial_{\mu} \theta(x)\right)\left(B^{\mu}+\partial^{\mu} \theta(x)\right) \\
& \neq \frac{1}{2} m_{\gamma} B_{\mu} B^{\mu}
\end{aligned}
$$

The above discussion was only for $U(1)$ and it can be extended to $S U(2)$ by simply replacing the constant parameter $\theta$ by $2 \times 2$ matrices(with determinant 1 ).

\subsection{Spontaneous Symmetry Breaking}

In the Standard Model (SM), the most general re-normalizable scalar potential for the Higgs field $(\operatorname{spin} 0$ ), which is a complex scalar field (with a hypercharge $Y=+1$ ) is [7]:

$$
V(\Phi)=-\mu^{2} \Phi^{\dagger} \Phi+\lambda\left(\Phi^{\dagger} \Phi\right)^{2}
$$

where $\Phi$ is a $S U(2)$ doublet and $S U(3)$ singlet. Then the Lagrangian for that field becomes,

$$
\mathcal{L}=\partial_{\mu} \Phi\left(\partial^{\mu} \Phi\right)^{\dagger}+\mu^{2} \Phi^{\dagger} \Phi-\lambda\left(\Phi^{\dagger} \Phi\right)^{2}
$$

To be precise, the derivative must be replaced by a covariant derivative in order to make the Lagrangian gauge invariant which will discussed in more detail in section 2.5. For the moment, the relevant piece is the scalar potential. Minimizing the scalar potential for the field, which means that it must satisfy

$$
\left.\frac{\partial V(\Phi)}{\partial \Phi}\right|_{\Phi_{0}}=0 \quad \text {, and }\left.\quad \frac{\partial^{2} V(\Phi)}{\partial \Phi^{2}}\right|_{\Phi_{0}}>0
$$


Applying these conditions to equation 2.28 gives:

$$
0=-2 \mu^{2}+4 \lambda \Phi_{0}^{2} \Longrightarrow \Phi_{0}=\sqrt{\frac{\mu^{2}}{2 \lambda}}
$$

In equation $2.31, \lambda$ is always taken as positive in order to make sure that the potential has an absolute minimum. If $\lambda$ is negative, then $V$ is unbounded from below and there is no stable vacuum. On the other hand, there are three possibilities for the sign of $\mu^{2}$.

Case 1: $\mu^{2}=0$

This is a trivial case, since it implies the $\left\langle\Phi_{0}\right\rangle=0$. Thus we don't have spontaneous symmetry breaking and all the particles in the SM will be massless.

Case 2: $\mu^{2}<0$

Investigating this particular case reveals that the minimum of the potentials occurs at $\left\langle\Phi_{0}\right\rangle=0$ as shown in figure 2.1. Thus the vacuum remains unchanged under the gauge transformation and the electroweak symmetry stays unbroken.

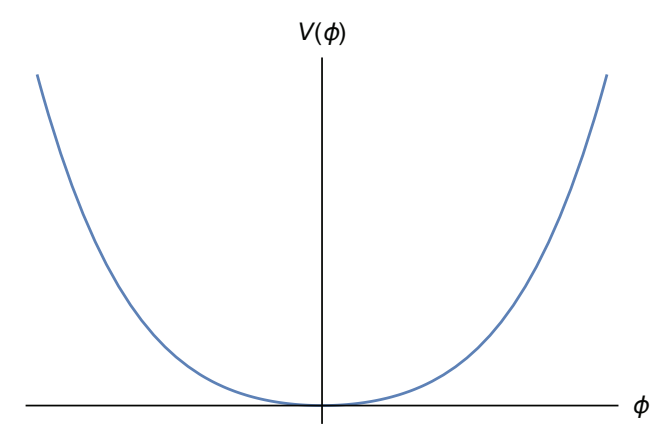

Figure 2.1: The Higgs potential as a function of the field for $\mu^{2}<0$.

Case 3: $\mu^{2}>0$ This is the most interesting case as shown in figure 2.2 because the vacuum state occurs away from zero. As a consequence, the electroweak symmetry 


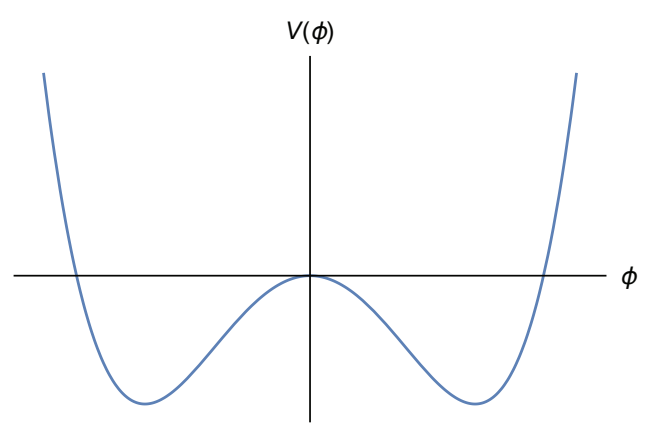

Figure 2.2: The Higgs potential as a function of the field for $\mu^{2}>0$.

$\left(S U(2)_{L} \times U(1)_{Y}\right)$ is broken. The minimum of the potential is colloquially called the vacuum expectation value (vev). Furthermore, it can be easily deduced from equation (2.31), that the minimization of the potential leads to the following fixed quantity ${ }^{1}$ :

$$
\Phi^{\dagger} \Phi=\frac{\mu^{2}}{2 \lambda}
$$

The Higgs field is a complex doublet, so it can be written in terms of four real scalar fields:

$$
\Phi=\left(\begin{array}{c}
\phi^{+} \\
\phi^{0}
\end{array}\right)=\frac{1}{\sqrt{2}}\left(\begin{array}{c}
\phi_{1}+i \phi_{2} \\
\phi_{3}+i \phi_{4}
\end{array}\right)
$$

Then expressing equation (2.32) in terms of the real fields produce,

$$
\Phi^{\dagger} \Phi=\frac{1}{2}\left(\phi_{1}^{2}+\phi_{2}^{2}+\phi_{3}^{2}+\phi_{4}^{2}\right)=\frac{\mu^{2}}{2 \lambda}
$$

Which is obviously a fixed length vector on a four dimensional spherical surface, so we are free to choose any basis in term of the real scalars as long as the condition in equation (2.34) is satisfied. For convenience, lets choose the vacuum expectation

\footnotetext{
${ }^{1}$ This is the vacuum value of the field $\Phi$, not what the field is always equal to.
} 
(denoted by $v$ ) of the fields to be:

$$
\left\langle\phi_{3}\right\rangle=\sqrt{\frac{\mu^{2}}{\lambda}} \equiv v \quad, \quad\left\langle\phi_{1}\right\rangle=\left\langle\phi_{2}\right\rangle=\left\langle\phi_{4}\right\rangle=0
$$

Thus it leads to

$$
\Phi_{0}=\frac{1}{\sqrt{2}}\left(\begin{array}{l}
0 \\
v
\end{array}\right)
$$

Now in order to determine the particle spectrum, we need to look at the perturbation around the minimum $(v)$. Hence, we have to introduce a new scalar field $h$ such that

$$
\phi_{3}=v+h \quad \text { with } \quad\langle h\rangle=0
$$

Rewriting the scalar potential in equation (2.28) in term of the real scalar fields defined above, one obtains

$$
\begin{aligned}
V & =-\frac{\mu^{2}}{2}\left((v+h)^{2}+\phi_{1}^{2}+\phi_{2}^{2}+\phi_{4}^{2}\right)+\frac{\lambda}{4}\left((v+h)^{2}+\phi_{1}^{2}+\phi_{2}^{2}+\phi_{4}^{2}\right)^{2} \\
& =\frac{\lambda h^{4}}{4}+\lambda v h^{3}+\lambda v^{2} h^{2}-\frac{\lambda v^{4}}{4}+\frac{\lambda h^{2} \phi_{2}^{2}}{2}+\lambda v h \phi_{2}^{2}+\frac{\lambda \phi_{2}^{4}}{4}+\frac{\lambda h^{2} \phi_{1}^{2}}{2} \\
& +\lambda v h \phi_{1}^{2}+\frac{\lambda \phi_{2}^{2} \phi_{1}^{2}}{2}+\frac{\lambda \phi_{1}^{4}}{4}+\frac{\lambda h^{2} \phi_{4}^{2}}{2}+\lambda v h \phi_{4}^{2}+\frac{\lambda \phi_{2}^{2} \phi_{4}^{2}}{2}+\frac{\lambda \phi_{1}^{2} \phi_{4}^{2}}{2}+\frac{\lambda \phi_{4}^{4}}{4}
\end{aligned}
$$

Notice that in the above expression, there are no mass terms (terms that are quadratic in the field, i.e $\left.\left(\phi_{i}\right)^{2}\right)$ for the scalar fields $\phi_{1}, \phi_{2}, \phi_{4}$ except $h$, which is $\lambda v^{2} h^{2}$. Hence, $\phi_{1}, \phi_{2}, \phi_{4}$ are massless. Now the general mass term for a real scalar field, lets say $\chi$, 
has the form $\frac{m_{\chi}^{2}}{2} \chi^{2}$, so the mass of the field $h$ is:

$$
\lambda v^{2} h^{2}=\frac{m_{h}^{2}}{2} h^{2} \Longrightarrow m_{h}=\sqrt{2 \lambda v^{2}}
$$

In QFT there is a general theorem, called Goldstone's theorem, about the massless mode that appears when there is spontaneous symmetry breaking. Goldstone's theorem states that for every spontaneous symmetry breaking, there must be a corresponding massless particle, called the Nambu-Goldstone boson, in the theory. In other words, for every broken generator of the global symmetry, there is a corresponding massless mode associated with it. Note that Goldstone's theorem applies to continuous global symmetries. If the fields are gauged away, then the massless modes are eaten by the gauge bosons corresponding to the broken generators and they become massive. In order to deal with the redundant degrees of freedom in the theory, one needs to fix a gauge, which is like choosing a particular coordinate system. So for convenience lets fix the gauge such that all the scalar fields except $\phi_{3}$ are set to zero at every point in spacetime. Hence we have,

$$
\Phi=\frac{1}{\sqrt{2}}\left(\begin{array}{c}
0 \\
h+v
\end{array}\right)
$$

This particular gauge choice is known as the unitary gauge. Naturally one wants to know how is the spontaneous symmetry breaking significant and how can it be used to understand nature? As such, lets explore some of its applications in the following sections. 


\subsection{Gauge Boson Masses}

From experimental observations, it has been determined that the observed gauge bosons $\left(W^{ \pm}\right.$and $\left.Z\right)$ are massive. So it is necessary that their masses be theoretically generated in agreement with the experimental observations. Before we obtain their masses, lets review some fundamental concepts. The Standard Model gauge sector is encoded in the direct product of groups $S U(3)_{c} \times S U(2)_{L} \times U(1)_{Y}$ and the detailed structure of these groups can be found in appendix A.2.

The gauge group of the SM, in addition to the $\mathrm{U}(1)$ contains the $\mathrm{SU}(2)$ group under which $\Phi$ transforms as:

$$
S U(2)_{L}: \Phi \longrightarrow \Phi^{\prime}=e^{\frac{1}{2} i \alpha_{j}(x) \sigma_{j}} \Phi
$$

where $\Phi$ is a two component vector, $\sigma_{j}$ are the Pauli matrices and $\alpha_{j}(x)$ are real parameters. So the transformation under the $S U(2)_{L} \times U(1)_{Y}$ gauge group is:

$$
S U(2)_{L} \times U(1)_{Y}: \Phi \longrightarrow \Phi^{\prime}=e^{\frac{1}{2} i \alpha_{j}(x) \sigma_{j}} e^{\frac{1}{2} i Y \beta(x)} \Phi
$$

Through the Higgs mechanism the above gauge group will lead to $S U(2)_{L} \times U(1)_{Y} \longrightarrow$

$U(1)_{E M}$. Recall, invariance under a transformation means that $e^{i \alpha_{j} \chi_{j}} \phi=\phi$ for the corresponding $\chi_{j}$ generator. For an infinitesimal transformation, one can expand the exponential in Taylor series (keep only the lowest order terms):

$$
e^{i \alpha_{j} \chi_{j}} \phi \approx\left(1+i \alpha_{j} \chi_{j}\right) \phi
$$


Thus invariance implies $\alpha_{j} \chi_{j} \phi=0$. So using equation 2.40:

$$
\begin{aligned}
U(1)_{Y}: Y \Phi_{0} & =\frac{Y}{\sqrt{2}}\left(\begin{array}{c}
0 \\
v+h
\end{array}\right) \\
S U(2)_{L}: \sigma_{1} \Phi_{0} & =\frac{1}{\sqrt{2}}\left(\begin{array}{cc}
0 & 1 \\
1 & 0
\end{array}\right)\left(\begin{array}{c}
0 \\
v+h
\end{array}\right)=\frac{1}{\sqrt{2}}\left(\begin{array}{c}
v+h \\
0
\end{array}\right) \\
\sigma_{2} \Phi_{0} & =\frac{1}{\sqrt{2}}\left(\begin{array}{cc}
0 & -i \\
i & 0
\end{array}\right)\left(\begin{array}{c}
0 \\
v+h
\end{array}\right)=\frac{-i}{\sqrt{2}}\left(\begin{array}{c}
v+h \\
0
\end{array}\right) \\
\sigma_{3} \Phi_{0} & =\frac{1}{\sqrt{2}}\left(\begin{array}{cc}
1 & 0 \\
0 & \\
0 & -1
\end{array}\right)\left(\begin{array}{c} 
\\
v+h
\end{array}\right)=\frac{-1}{\sqrt{2}}\left(\begin{array}{c}
0 \\
v+h
\end{array}\right)
\end{aligned}
$$

However, with the choice of hypercharge $Y=+1$, the following quantity leaves the vacuum state invariant:

$$
\begin{aligned}
Q \Phi_{0} & =\frac{1}{2}\left(Y+\sigma_{3}\right) \Phi_{0}=\frac{1}{2 \sqrt{2}}\left(\mathbb{I}_{2 \times 2}+\sigma_{3}\right)\left(\begin{array}{c}
0 \\
v+h
\end{array}\right) \\
& =\frac{1}{\sqrt{2}}\left(\begin{array}{cc}
1 & 0 \\
0 & 0
\end{array}\right)\left(\begin{array}{c}
0 \\
v+h
\end{array}\right)=0
\end{aligned}
$$

So the expression relating the weak hypercharge and the electric charge is,

$$
Q=\frac{1}{2} Y+T_{3}=\frac{1}{2}\left(Y+\sigma_{3}\right)
$$


where $T_{3}$ is the third component of the weak isospin. Consequently, all the generators of $S U(2)_{L}$ and $U(1)_{Y}$ are broken in our chosen vacuum(equation 2.40). Since the symmetry is gauged, the three goldstone bosons are eaten by the gauge bosons corresponding to the broken generators. So the SM gauge bosons $\left(W^{ \pm}\right.$and $\left.Z\right)$ acquire masses through the Higgs mechanism. In order to make the Lagrangian invariant under $S U(2)_{L} \times U(1)_{Y}$, then one needs to add an extra term to the covariant derivative (equation 2.24) to compensate for the $S U(2)_{L}$ gauge transformation. So the covariant derivative with the corresponding gauge fields infinitesimal transformation is [7],

$$
\begin{array}{r}
D_{\mu}=\partial_{\mu}-\frac{i}{2} g Y B_{\mu}-\frac{i}{2} g_{w} W_{\mu}^{j} \sigma^{j} \\
B_{\mu} \longrightarrow B_{\mu}^{\prime}=B_{\mu}+\frac{1}{g} \partial_{\mu} \beta(x) \\
W_{\mu}^{j} \longrightarrow\left(W_{\mu}^{j}\right)^{\prime}=W_{\mu}^{j}+\frac{1}{g_{w}} \partial_{\mu} \alpha^{j}(x)+\epsilon^{j k l} W_{\mu}^{k} \alpha^{l}
\end{array}
$$

Hypercharge $Y=+1$ is assigned to the field $\Phi$. Then the gauge boson mass term 
comes from expanding the kinetic term of the field $\Phi$ at the vacuum. So the appropriate terms are,

$$
\begin{aligned}
& \Delta \mathcal{L}=\left(D_{\mu} \Phi_{0}\right)^{\dagger}\left(D^{\mu} \Phi_{0}\right) \\
& =\frac{1}{2}\left(\begin{array}{ll}
0 & v+h
\end{array}\right)\left(\partial_{\mu}+\frac{1}{2} i g Y B_{\mu}+i \frac{1}{2} g_{w} W_{\mu}^{j} \sigma^{j}\right)\left(\partial_{\mu}-\frac{1}{2} i g Y B^{\mu}-i \frac{1}{2} g_{w} W^{\mu j} \sigma^{j}\right)\left(\begin{array}{c}
0 \\
v+h
\end{array}\right) \\
& =\frac{1}{2}\left(\begin{array}{ll}
0 & v+h
\end{array}\right)\left(\begin{array}{cc}
\partial_{\mu}+i \frac{g_{w}}{2} W_{\mu}^{3}+\frac{1}{2} i g Y B_{\mu} & i \frac{g_{w}}{2}\left(W_{\mu}^{1}-i W_{\mu}^{2}\right) \\
i \frac{g_{w}}{2}\left(W_{\mu}^{1}+i W_{\mu}^{2}\right) & \partial_{\mu}+\frac{1}{2} i g Y B_{\mu}-i \frac{g_{w}}{2} W_{\mu}^{3}
\end{array}\right) . \\
& \left(\begin{array}{cc}
\partial_{\mu}-i \frac{g_{w}}{2} W^{3 \mu}-\frac{1}{2} i g Y B^{\mu} & -i \frac{g_{w}}{2}\left(W^{1 \mu}-i W^{2 \mu}\right) \\
-i \frac{g_{w}}{2}\left(W^{1 \mu}+i W^{2 \mu}\right) & \partial_{\mu}-\frac{1}{2} i g Y B^{\mu}+i \frac{g_{w}}{2} W^{3 \mu}
\end{array}\right)\left(\begin{array}{c}
0 \\
v+h
\end{array}\right) \\
& =\frac{1}{2}\left(\frac{i g_{w}}{2}\left(W_{\mu}^{1}+i W_{\mu}^{2}\right) \quad \partial_{\mu}+\frac{1}{2} i g Y B_{\mu}-i \frac{g_{w}}{2} W_{\mu}^{3}\right)(v+h)\left(\begin{array}{c}
\frac{-i g_{w}}{2}\left(W^{1 \mu}-i W^{2 \mu}\right) \\
\partial_{\mu}-\frac{1}{2} i g Y B^{\mu}+i \frac{g_{w}}{2} W^{3 \mu}
\end{array}\right)(v+h) \\
& =\frac{1}{2} \partial_{\mu} h \partial^{\mu} h+\frac{(v+h)^{2}}{2}\left\{\frac{g_{w}^{2}}{4}\left[\left(W_{\mu}^{1}\right)^{2}+\left(W_{\mu}^{2}\right)^{2}\right]+\frac{1}{4}\left(g_{w} W_{\mu}^{3}-g Y B_{\mu}\right)^{2}\right\}
\end{aligned}
$$

Rewriting the last term:

$$
\left(g_{w} W_{\mu}^{3}-g Y B_{\mu}\right)^{2}=\left(\begin{array}{ll}
W_{\mu}^{3} & B_{\mu}
\end{array}\right)\left(\begin{array}{cc}
g_{w}^{2} & -g g_{w} Y \\
-g g_{w} Y & g^{2} Y^{2}
\end{array}\right)\left(\begin{array}{c}
W_{\mu}^{3} \\
B_{\mu}
\end{array}\right)
$$

So diagonalizing the above matrix with $Y=+1$, the eigenvalues are:

$$
\lambda=0, \quad \lambda=g^{2}+g_{w}^{2}
$$


Then the corresponding normalized eigenvectors $(A \cdot \boldsymbol{V}=\lambda \boldsymbol{V})$ are:

$$
\begin{aligned}
& A_{\mu} \equiv V_{1}=\frac{1}{\sqrt{g^{2}+g_{w}^{2}}}\left(g W_{\mu}^{3}+g_{w} B_{\mu}\right) \\
& Z_{\mu} \equiv V_{2}=\frac{1}{\sqrt{g^{2}+g_{w}^{2}}}\left(g_{w} W_{\mu}^{3}-g B_{\mu}\right)
\end{aligned}
$$

Thus rewriting the last term in the Lagrangian, equation 2.48 in terms of the mass basis, we arrive at:

$$
\begin{aligned}
\Delta \mathcal{L} & \supset \frac{(v+h)^{2}}{2} \frac{1}{4}\left(g_{w} W_{\mu}^{3}-g Y B_{\mu}\right)^{2}=\frac{(v+h)^{2}}{8}\left(g^{2}+g_{w}^{2}\right) Z_{\mu} Z^{\mu} \\
& =\frac{\left(g^{2}+g_{w}^{2}\right) v^{2}}{8} Z_{\mu} Z^{\mu}+\frac{\left(g^{2}+g_{w}^{2}\right) v}{4} h Z_{\mu} Z^{\mu}+\frac{\left(g^{2}+g_{w}^{2}\right)}{8} h^{2} Z_{\mu} Z^{\mu}
\end{aligned}
$$

The first term is the $Z$ boson's mass term and the other two describe various interactions with the Higgs field. Hence the $Z$ boson's mass is [8]:

$$
\frac{1}{2} m_{Z}^{2} Z_{\mu} Z^{\mu}=\frac{\left(g^{2}+g_{w}^{2}\right) v^{2}}{8} Z_{\mu} Z^{\mu} \Longrightarrow m_{Z}=\frac{\sqrt{g^{2}+g_{w}^{2}} v}{2}
$$

Furthermore, rewriting $W_{\mu}^{1}$ and $W_{\mu}^{2}$ in terms of the mass eigenstates $W_{\mu}^{+}$and $W_{\mu}^{-}$:

$$
W_{\mu}^{ \pm} \equiv \frac{1}{\sqrt{2}}\left(W_{\mu}^{1} \mp i W_{\mu}^{2}\right)
$$

then the middle term in equation 2.48 gives,

$$
\begin{aligned}
\Delta \mathcal{L} & \supset \frac{(v+h)^{2}}{2}\left\{\frac{g_{w}^{2}}{4}\left[\left(W_{\mu}^{1}\right)^{2}+\left(W_{\mu}^{2}\right)^{2}\right]\right\}=\frac{(v+h)^{2} g_{w}^{2}}{8}\left[\left(W_{\mu}^{+}\right)^{2}+\left(W_{\mu}^{-}\right)^{2}\right] \\
& =\frac{v^{2} g_{w}^{2}}{8}\left[\left(W_{\mu}^{+}\right)^{2}+\left(W_{\mu}^{-}\right)^{2}\right]+\frac{v g_{w}^{2}}{4} h\left[\left(W_{\mu}^{+}\right)^{2}+\left(W_{\mu}^{-}\right)^{2}\right]+\frac{g_{w}^{2}}{8} h^{2}\left[\left(W_{\mu}^{+}\right)^{2}+\left(W_{\mu}^{-}\right)^{2}\right]
\end{aligned}
$$

Thus the first term results in the masses of $W^{ \pm}$bosons, and the remaining terms 
shows the various type of $W$ boson interactions with the Higgs field. So the $W$ boson mass is [8]:

$$
\frac{1}{2} m_{W^{ \pm}}^{2}=\frac{v^{2} g_{w}^{2}}{8} \Longrightarrow m_{W^{ \pm}}=\frac{v g_{w}}{2}
$$

Note the photon remains massless because there is no mass term in the Lagrangian (a term $\propto A_{\mu} A^{\mu}$ ) as expected. This generation scheme is known as the Higgs mechanism in particle physics. So far we have only been concerned with the gauge sector but what about the leptons of the SM?

\subsection{Lepton Masses}

In general, there are two known types of fermion masses, Majorana and Dirac fermions. Majorana fermions are their own antiparticle and in contrast, the Dirac fermions are not. In the Dirac representation, the fermion field is a four component spinor. However, using the Weyl bi-spinors one can write it as follows:

$$
\psi=\left(\begin{array}{c}
\psi_{L} \\
\psi_{R}
\end{array}\right)
$$

where $\psi_{L}$ and $\psi_{R}$ are two components object called the left and right handed Weyl spinors respectively. These left and right handed chiral fermions states (definite handedness) can be obtained from $\psi$ using the projection operators,

$$
\begin{aligned}
& P_{L} \equiv \frac{1}{2}\left(1-\gamma^{5}\right) \quad P_{R} \equiv \frac{1}{2}\left(1+\gamma^{5}\right) \\
& P_{L}+P_{R}=1, \quad P_{L} P_{R}=P_{R} P_{L}=0, \quad P_{L}^{2}=P_{L}, \quad P_{R}^{2}=P_{R}
\end{aligned}
$$


Then,

$$
\begin{aligned}
& \psi_{L}=P_{L} \psi=\frac{1}{2}\left(1-\gamma^{5}\right) \psi \\
& \psi_{R}=P_{R} \psi=\frac{1}{2}\left(1+\gamma^{5}\right) \psi
\end{aligned}
$$

One can further derive:

$$
\begin{aligned}
\gamma^{5} P_{L} & =\gamma^{5} \frac{1}{2}\left(1-\gamma^{5}\right)=-P_{L}, \quad \gamma^{\mu} P_{L}=P_{R} \gamma^{\mu} \\
\gamma^{5} P_{R} & =\gamma^{5} \frac{1}{2}\left(1+\gamma^{5}\right)=P_{R}
\end{aligned}
$$

Before one writes down the Lagrangian for Dirac fermions, one needs to figure out how to construct a Lorentz scalar quantity using $\psi$. A quantity of the form $\psi^{\dagger} \psi$ is not Lorentz scalar because the Lorentz transformation is not unitary but $\bar{\psi} \psi$ is [7]:

$$
\begin{aligned}
& \bar{\psi} \equiv \psi^{\dagger} \gamma^{0} \\
& \psi \stackrel{\text { Lorentz }}{\longrightarrow} \psi^{\prime}=\Lambda_{\frac{1}{2}} \psi \\
& \bar{\psi} \stackrel{\text { Lorentz }}{\longrightarrow} \overline{\psi^{\prime}}=\bar{\psi} \Lambda_{\frac{1}{2}}^{-1}, \quad \text { then } \\
& \bar{\psi} \psi \stackrel{\text { Lorentz }}{\longrightarrow} \overline{\psi^{\prime}} \psi^{\prime}=\bar{\psi} \Lambda_{\frac{1}{2}}^{-1} \Lambda_{\frac{1}{2}} \psi=\bar{\psi} \psi
\end{aligned}
$$

where,

$$
\Lambda_{\frac{1}{2}}=\exp \left(-\frac{i}{2} \omega_{\mu \nu} S^{\mu \nu}\right), \quad \text { with } S^{\mu \nu}=\frac{i}{4}\left[\gamma^{\mu}, \gamma^{\nu}\right]
$$

With most of the relevant information in hand, one can then go ahead and come up with a Lorentz invariant Lagrangian for a free Dirac particle as:

$$
\mathcal{L}=i \bar{\psi} \gamma^{\mu} \partial_{\mu} \psi-m \bar{\psi} \psi
$$


This is correct because the Euler-Lagrange equation leads to the Dirac equation:

$$
i \gamma^{\mu} \partial_{\mu} \psi-m \psi=0
$$

However, we run into a problem when we require that the Lagrangian in equation 2.63 be invariant under the gauge transformation $S U(2)_{L} \times U(1)_{Y}$. Indeed, it was experimentally established that the left and right handed spinors of all the SM fermions have different transformation properties. The difficulty arises from the mass term $m \bar{\psi} \psi$ which will become obvious when it is written in terms of Weyl spinors.

$$
\begin{aligned}
\mathcal{L} & =i \bar{\psi} \gamma^{\mu} \partial_{\mu} \psi-m \bar{\psi} \psi \\
& =i \bar{\psi} \gamma^{\mu}\left(P_{L} P_{L}+P_{R} P_{R}\right) \partial_{\mu} \psi-m \bar{\psi}\left(P_{L} P_{L}+P_{R} P_{R}\right) \psi \\
& =i \bar{\psi}_{L} \gamma^{\mu} \partial_{\mu} \psi_{L}+i \bar{\psi}_{R} \gamma^{\mu} \partial_{\mu} \psi_{R}-m\left(\bar{\psi}_{R} \psi_{L}+\bar{\psi}_{L} \psi_{R}\right)
\end{aligned}
$$

Now the kinetic terms are fine since fields of the same chirality appear as a pair so one only needs to change $\partial_{\mu} \longrightarrow D_{\mu}$ to make them gauge invariant. However, the mass terms have fields of different chiralities. This is a concern because the right handed spinors is singlet under $S U(2)_{L}$ transformation (i.e: $\psi_{R} \stackrel{S U(2)_{L}}{\longrightarrow} \psi_{R}^{\prime}=e^{\frac{i}{2} \alpha_{j} \sigma_{j}} \psi_{R}=\psi_{R}$ ) and it carries a different gauge charge than the left handed spinors which are doublets under $S U(2)_{L}$ transformation(i.e: $\left.\psi_{L} \stackrel{S U(2)_{L}}{\longrightarrow} \psi_{L}^{\prime}=e^{\frac{i}{2} \alpha_{j} \sigma_{j}} \psi_{L} \approx\left[1+\frac{i}{2} \alpha_{j} \sigma_{j}\right] \psi_{L}\right)$. Thus a mass term of that form breaks the gauge invariance and is thus forbidden due to gauge transformation. Since the observed fermions have masses; then, their masses must come from coupling to the Higgs field. The fermions mass terms are allowed by coupling to the Higgs field because the Higgs field is charged under $S U(2)_{L}$ and a gauge invariant term, of the form given in equation 2.66, can be written for the leptons.

In order to obtain masses from interaction with the Higgs field, Lorentz invariance 
requires that the Dirac field must come in pairs (i.e: $\bar{\psi} \psi$ ). Now $\Phi$ is an $S U(2)_{L}$ doublet, so gauge invariance demands that it has to be coupled to a fermion field that is also an $S U(2)_{L}$ doublet. Thus a normalizable term must contain two $S U(2)_{L}$ doublets (one $\Phi$ and one $\psi_{L}$ ) and one $S U(2)_{L}$ singlet $\left(\psi_{R}\right)$. So the Yukawa interaction for the first generation of leptons (we ignore generating masses for the neutrinos) is:

$$
\begin{aligned}
\mathcal{L}_{\text {Yukawa }} \supset-\lambda_{e}\left(\bar{e}_{R} \Phi^{\dagger} L_{L}+\bar{L}_{L} \Phi e_{R}\right), \quad \text { where } L_{L}=\left(\begin{array}{l}
\nu_{e} \\
e
\end{array}\right)_{L} \\
=-\lambda_{e}\left[\frac{1}{\sqrt{2}} \bar{e}_{R}\left(\begin{array}{ll}
0 & v+h
\end{array}\right)\left(\begin{array}{l}
\nu_{e} \\
e
\end{array}\right)_{L}+\frac{1}{\sqrt{2}}\left(\begin{array}{ll}
\bar{\nu}_{e} & \bar{e}
\end{array}\right)_{L}\left(\begin{array}{c}
0 \\
v+h
\end{array}\right) e_{R}\right] \\
=-\frac{1}{\sqrt{2}} \lambda_{e}(v+h)\left[\bar{e}_{R} e_{L}+\bar{e}_{L} e_{R}\right]=-\frac{1}{\sqrt{2}} \lambda_{e}(v+h) \bar{e} e \\
=-\frac{1}{\sqrt{2}} \lambda_{e} v \bar{e} e-\frac{1}{\sqrt{2}} \lambda_{e} h \bar{e} e
\end{aligned}
$$

The first term in equation 2.66 is the electron mass term and the other describes the electron interaction with the Higgs field. Consequently the electron mass is:

$$
m_{e} \bar{e} e=\frac{1}{\sqrt{2}} \lambda_{e} v \bar{e} e \Longrightarrow m_{e}=\frac{1}{\sqrt{2}} \lambda_{e} v
$$

We can generalize this to include all three generation of leptons, so the general socalled Yukawa type interactions for leptons can be written as:

$$
\mathcal{L}_{\text {Yukawa }}^{\text {leptons }} \supset-\sum_{i=1}^{3}\left(\lambda_{l}^{i} \bar{L}_{L}^{i} \Phi e_{R}^{i}+\text { h.c. }\right)
$$

where the index $i$ represent the different generations $(e, \mu, \tau)$. 


\subsection{Quark Masses}

Similarly to leptons, the quark masses are generated by the Higgs field through Yukawa type interactions. So by the same logic as before, one can write the Yukawa terms for only the first generation down type quarks as:

$$
\begin{aligned}
& \mathcal{L}_{\text {Yukawa }} \supset-\left(\lambda_{d} \bar{d}_{R} \Phi^{\dagger} Q_{L}+\lambda_{d}^{*} \bar{Q}_{L} \Phi d_{R}\right), \quad \text { where } Q_{L}=\left(\begin{array}{l}
u \\
\\
d
\end{array}\right)_{L} \\
& =-\left[\lambda_{d} \frac{1}{\sqrt{2}} \bar{d}_{R}\left(\begin{array}{ll}
0 & v+h
\end{array}\right)\left(\begin{array}{l}
u \\
d
\end{array}\right)_{L}+\lambda_{d}^{*} \frac{1}{\sqrt{2}}\left(\begin{array}{ll}
\bar{u} & \bar{d}
\end{array}\right)_{L}\left(\begin{array}{c}
0 \\
v+h
\end{array}\right] d_{R}\right] \\
& =-\frac{1}{\sqrt{2}}(v+h)\left[\lambda_{d} \bar{d}_{R} d_{L}+\lambda_{d}^{*} \bar{d}_{L} d_{R}\right] \quad, \quad \text { Assume } \lambda_{d} \text { is real } \\
& =-\frac{1}{\sqrt{2}} \lambda_{d}(v+h) \bar{d} d=-\frac{1}{\sqrt{2}} \lambda_{d} v \bar{d} d-\frac{1}{\sqrt{2}} \lambda_{d} h \bar{d} d
\end{aligned}
$$

Hence the mass for the down quark is:

$$
m_{d}=\frac{1}{\sqrt{2}} \lambda_{d} v
$$

Note that we can only generate masses for the down type quarks by using the term of the form $\bar{\psi}_{L} \Phi \psi_{R}$. In order to generate masses for the up-type quarks, one needs to add a different kind of term to the Lagrangian. First one defines a rotated field 
(anti-doublet) as:

$$
\begin{array}{r}
\tilde{\Phi} \equiv i \sigma^{2} \Phi^{*}=i\left(\begin{array}{cc}
0 & -i \\
i & 0
\end{array}\right)\left(\begin{array}{c}
\phi^{+*} \\
\phi^{0 *}
\end{array}\right)=\left(\begin{array}{c}
\phi^{0 *} \\
-\phi^{+*}
\end{array}\right) \\
\text { so in the unitary gauge } \Longrightarrow \tilde{\Phi} \equiv i \sigma^{2} \Phi^{*}=\frac{1}{\sqrt{2}}\left(\begin{array}{c}
v+h \\
0
\end{array}\right)
\end{array}
$$

Taking advantage of this field, one can come up with the following Yukawa terms for the first generation of the up-type quark as:

$$
\begin{aligned}
\mathcal{L}_{\text {Yukawa }} & \supset-\left(\lambda_{u} \bar{u}_{R} \tilde{\Phi}^{\dagger} Q_{L}+\lambda_{u}^{*} \bar{Q}_{L} \tilde{\Phi} u_{R}\right) \\
& =-\frac{1}{\sqrt{2}}(v+h) \lambda_{u} \bar{u} u \Longrightarrow m_{u}=\frac{1}{\sqrt{2}} \lambda_{u} v
\end{aligned}
$$

The above expressions only describe the first generation of the quark sector, so it must be extended to include all three generations for completeness. Consequently, the general Yukawa Lagrangian for the quarks is:

$$
\mathcal{L}_{\text {Yukawa }}^{\text {quarks }} \supset-\sum_{j=1}^{3} \sum_{k=1}^{3}\left[\lambda_{u}^{j k} \bar{u}_{R}^{\prime j} \tilde{\Phi}^{\dagger} Q_{L}^{\prime k}+\lambda_{d}^{j k} \bar{d}_{R}^{\prime j} \Phi^{\dagger} Q_{L}^{\prime k}+\text { h.c. }\right]
$$

In general, $\lambda_{u}$ and $\lambda_{d}$ are matrices which results in mixing between different flavours of quarks ${ }^{2}$. Also remember all the quarks are $S U(3)_{c}$ triplets so to preserve gauge invariance, it is compulsory to modify the covariant derivative. The quark fields are not mass eigenstates because the matrices in equation 2.73 give mixing terms for different generations of the quark fields. One needs to rotate these fields into the mass basis for which the mass matrices are diagonal. To obtain the mass eigenstates,

\footnotetext{
${ }^{2} \mathrm{~A}$ similar type of mixing also happens for the neutrinos but not for the charged leptons.
} 
one can use unitary matrices to diagonalize $\lambda_{u}$ and $\lambda_{d}$ matrices. The mass eigenstates are given by:

$$
\begin{array}{r}
u_{L, R}^{\prime j}=U_{L, R}^{j k} u_{L, R}^{k}, \quad d_{L, R}^{\prime j}=D_{L, R}^{j k} d_{L, R}^{k} \\
\text { where } u_{L, R}=\left(\begin{array}{c}
u \\
c \\
t
\end{array}\right)_{L, R}, d_{L, R}=\left(\begin{array}{l}
d \\
s \\
b
\end{array}\right)_{L, R} \\
U_{L, R} U_{L, R}^{\dagger}=U_{L, R}^{\dagger} U_{L, R}=\mathbb{I}, \quad D_{L, R} D_{L, R}^{\dagger}=D_{L, R}^{\dagger} D_{L, R}=\mathbb{I}
\end{array}
$$

Then the diagonal quark mass matrices are given by:

$$
\begin{aligned}
M_{d}^{\text {diagonal }} \equiv & \left(\begin{array}{ccc}
m_{d} & 0 & 0 \\
0 & m_{s} & 0 \\
0 & 0 & m_{b}
\end{array}\right) \equiv \frac{v}{\sqrt{2}} D_{R}^{\dagger} \lambda_{d} D_{L} \\
M_{u}^{\text {diagonal }} & \equiv\left(\begin{array}{ccc}
m_{u} & 0 & 0 \\
0 & m_{c} & 0 \\
0 & 0 & m_{t}
\end{array}\right) \equiv \frac{v}{\sqrt{2}} U_{R}^{\dagger} \lambda_{u} U_{L}
\end{aligned}
$$

As a result of these rotations, the mass eigenstates for the up-type quarks are no longer matched with the down-type quarks within the same doublet. This adds further complexities to the SM since this will allow for flavour changing interactions. 


\subsection{Kinetic Terms}

In this section we examine the kinetic terms of various fields of the SM. Based on the previous discussions, we now know that for spin $\frac{1}{2}$ Dirac fermions the kinetic terms takes the form $i \bar{\psi} \gamma^{\mu} \partial_{\mu} \psi$. It can be written in a more compact way by absorbing the Dirac matrices into the derivative operator. So lets define the Feynman slash notation:

$$
\not \partial_{\mu} \equiv \gamma^{\mu} \partial_{\mu}, \quad \not P_{\mu} \equiv \gamma^{\mu} P_{\mu}, \quad \not B_{\mu} \equiv \gamma^{\mu} B_{\mu}
$$

Then by imposing gauge invariance, it is compulsory to change $\partial_{\mu} \longrightarrow D_{\mu}$ and note that there are gauge fields coming from the various groups $\left(S U(3)_{c} \times S U(2)_{L} \times U(1)_{Y}\right)$. For $U(1)_{Y}$, the associated gauge fields is $B_{\mu}$, which has the corresponding kinetic term:

$$
\mathcal{L}_{Q E D} \supset-\frac{1}{4} F_{\mu \nu} F^{\mu \nu}
$$

where the field strength is $F_{\mu \nu} \equiv \partial_{\mu} B_{\nu}-\partial_{\nu} B_{\mu}$

Similarly, one comes up with gauge kinetic terms for the remaining gauge fields as [9]:

$$
\mathcal{L}_{\text {weak }}=-\frac{1}{4} W_{\mu \nu}^{a} W^{a \mu \nu}, \quad a=1,2,3
$$

where, $\quad W_{\mu \nu}^{a}=\partial_{\mu} W_{\nu}^{a}-\partial_{\nu} W_{\mu}^{a}+g_{w} \epsilon^{a j k} W_{\mu}^{j} W_{\nu}^{k}$

$$
\mathcal{L}_{Q C D}=-\frac{1}{4} G_{\mu \nu}^{a} G^{a \mu \nu}, \quad a=1,2, \ldots, 8
$$

where $, G_{\mu \nu}^{a}=\partial_{\mu} G_{\nu}^{a}-\partial_{\nu} G_{\mu}^{a}+g_{s} f^{a j k} G_{\mu}^{j} G_{\nu}^{k}, \quad j, k=1,2, \ldots, 8$

where $f^{i j k}$ is the structure constant of $S U(3)$ 
Thus the complete gauge kinetic term is:

$$
\mathcal{L}_{\text {gauge }}=-\frac{1}{4} F_{\mu \nu} F^{\mu \nu}-\frac{1}{4} W_{\mu \nu}^{a} W^{a \mu \nu}-\frac{1}{4} G_{\mu \nu}^{a} G^{a \mu \nu}
$$

Now investigating the kinetic terms for the Dirac fermions and using the information from equation 2.65 , one can write a gauge invariant expression for only one generation of leptons and quarks as:

$$
\mathcal{L}_{\text {kinetic }}^{1} \supset i \bar{L}_{L} \not D L_{L}+i \bar{e}_{R} \not D e_{R}+i \bar{Q}_{L} \not D Q_{L}+i \bar{u}_{R} \not D u_{R}+i \bar{d}_{R} \not D d_{R}
$$

Consequently, the kinetic energy term for all the generations of fermions can be written as:

$$
\begin{array}{r}
\mathcal{L}_{\text {kinetic }}=i \sum_{j=1}^{3}\left(\bar{L}_{L}^{j} \not D L_{L}^{j}+\bar{e}_{R}^{j} \not D e_{R}^{j}+\bar{Q}_{L}^{j} \not D Q_{L}^{j}+\bar{u}_{R}^{j} \not D u_{R}^{j}+\bar{d}_{R}^{j} \not D d_{R}^{j}\right) \\
\text { Recall, } e_{R}^{j}=\left(e_{R}, \mu_{R}, \tau_{R}\right), u_{R}^{j}=\left(u_{R}, c_{R}, t_{R}\right), d_{R}=\left(d_{R}, s_{R}, b_{R}\right) \\
Q_{L}^{j}=\left[\left(\begin{array}{l}
u^{\prime} \\
d^{\prime}
\end{array}\right)_{L},\left(\begin{array}{c}
c^{\prime} \\
s^{\prime}
\end{array}\right)_{L},\left(\begin{array}{l}
t^{\prime} \\
b^{\prime}
\end{array}\right)_{L}\right], L_{L}^{j}=\left[\left(\begin{array}{l}
\nu_{e} \\
e
\end{array}\right)_{L},\left(\begin{array}{l}
\nu_{\mu} \\
\mu
\end{array}\right)_{L},\left(\begin{array}{l}
\nu_{\tau} \\
\tau
\end{array}\right)_{L}\right]
\end{array}
$$

With the entire SM in sight, one can move further to examine the kinetic term for one generation of quarks and observe that the flavour changing interactions transpire from the weak bosons exchange. Before we show this, lets first rewrite the covariant derivative for the electroweak case in terms of the mass eigenstates. In order to 
accomplish this, lets use equation 2.51,

$$
\begin{aligned}
A_{\mu} & \equiv \frac{1}{\sqrt{g^{2}+g_{w}^{2}}}\left(g W_{\mu}^{3}+g_{w} B_{\mu}\right), \quad Z_{\mu} \equiv \frac{1}{\sqrt{g^{2}+g_{w}^{2}}}\left(g_{w} W_{\mu}^{3}-g B_{\mu}\right) \\
\Longrightarrow B_{\mu} & =\frac{1}{\sqrt{g^{2}+g_{w}^{2}}}\left(g_{w} A_{\mu}-g Z_{\mu}\right)=c_{W} A_{\mu}-s_{W} Z_{\mu} \\
W_{\mu}^{3} & =\frac{1}{\sqrt{g^{2}+g_{w}^{2}}}\left(g A_{\mu}+g_{w} Z_{\mu}\right)=s_{W} A_{\mu}+c_{W} Z_{\mu}
\end{aligned}
$$

where , $c_{W}=\frac{g_{w}}{\sqrt{g^{2}+g_{w}^{2}}}, \quad s_{W}=\frac{g}{\sqrt{g^{2}+g_{w}^{2}}}$

Furthermore we can write,

$$
\begin{aligned}
W_{\mu}^{1} \sigma^{1}+W_{\mu}^{2} \sigma^{2} & =\frac{1}{2}\left(W_{\mu}^{1}+i W_{\mu}^{2}\right)\left(\sigma^{1}-i \sigma^{2}\right)+\frac{1}{2}\left(W_{\mu}^{1}-i W_{\mu}^{2}\right)\left(\sigma^{1}+i \sigma^{2}\right) \\
& =\frac{1}{\sqrt{2}} W_{\mu}^{-}\left(\sigma^{1}-i \sigma^{2}\right)+\frac{1}{\sqrt{2}} W_{\mu}^{+}\left(\sigma^{1}+i \sigma^{2}\right)=\sqrt{2}\left(W_{\mu}^{-} T^{-}+W_{\mu}^{+} T^{+}\right) \\
\text {where }, T^{+} & =\frac{1}{2}\left(\sigma^{1}+i \sigma^{2}\right) \text { and } \quad T^{-}=\frac{1}{2}\left(\sigma^{1}-i \sigma^{2}\right), \quad T^{3}=\frac{1}{2} \sigma^{3}
\end{aligned}
$$

Using the above established notations, one obtains:

$$
\begin{aligned}
D_{\mu} & =\partial_{\mu}-\frac{i}{2} g Y B_{\mu}-\frac{i}{2} g_{w} W_{\mu}^{j} \sigma^{j} \\
& =\partial_{\mu}-\frac{i}{2} g Y\left(c_{W} A_{\mu}-s_{W} Z_{\mu}\right)-\frac{i}{2} g_{w} \sqrt{2}\left(W_{\mu}^{-} T^{-}+W_{\mu}^{+} T^{+}\right)-i g_{w} W_{\mu}^{3} T^{3} \\
& =\partial_{\mu}-\frac{i}{2} g Y\left(c_{W} A_{\mu}-s_{W} Z_{\mu}\right)-\frac{i}{\sqrt{2}} g_{w}\left(W_{\mu}^{-} T^{-}+W_{\mu}^{+} T^{+}\right)-i g_{w}\left(s_{W} A_{\mu}+c_{W} Z_{\mu}\right) T^{3} \\
& =\partial_{\mu}-i\left(\frac{1}{2} g c_{W} Y+g_{w} s_{W} T^{3}\right) A_{\mu}-i\left(g_{w} c_{W} T^{3}-\frac{1}{2} g s_{W} Y\right) Z_{\mu}-\frac{i}{\sqrt{2}} g_{w}\left(W_{\mu}^{-} T^{-}+W_{\mu}^{+} T^{+}\right) \\
& =\partial_{\mu}-i e Q A_{\mu}-i\left(g_{w} c_{W} T^{3}-\frac{1}{2} g s_{W} Y\right) Z_{\mu}-\frac{i}{\sqrt{2}} g_{w}\left(W_{\mu}^{-} T^{-}+W_{\mu}^{+} T^{+}\right) \\
& \text {where, } Q \equiv T^{3}+\frac{1}{2} Y, \quad e \equiv g c_{W}=\frac{g g_{w}}{\sqrt{g^{2}+g_{w}^{2}}}
\end{aligned}
$$

With all the pieces in place, we can use the above expression to write down the quarks 
kinetic term as follow:

$$
\begin{aligned}
\mathcal{L}_{\text {kinetic }}^{F C} & =i \bar{Q}_{L}^{j} \not D Q_{L}^{j} \\
& =i \bar{Q}_{L}^{j} \gamma^{\mu}\left[\partial_{\mu}-i e Q A_{\mu}-i\left(g_{w} c_{W} T^{3}-\frac{1}{2} g s_{W} Y\right) Z_{\mu}-\frac{i}{\sqrt{2}} g_{w}\left(W_{\mu}^{-} T^{-}+W_{\mu}^{+} T^{+}\right)-\frac{i}{2} g_{s} G_{\mu}^{a} \lambda^{a}\right] Q_{L}^{j} \\
& =i \bar{Q}_{L}^{j} \not{ }_{\mu} Q_{L}^{j}+e Q \bar{Q}_{L}^{j} A_{\mu} Q_{L}^{j}+\left(g_{w} c_{W} T^{3}-\frac{1}{2} g s_{W} Y\right) \bar{Q}_{L}^{j} \boldsymbol{L}_{\mu} Q_{L}^{j}+\frac{1}{\sqrt{2}} g_{w} \bar{Q}_{L}^{j} W_{\mu}^{-} T^{-} Q_{L}^{j}+ \\
& \frac{1}{\sqrt{2}} g_{w} \bar{Q}_{L}^{j} W_{\mu}^{+} T^{+} Q_{L}^{j}+\frac{1}{2} g_{s} \bar{Q}_{L}^{j} G_{\mu}^{a} \lambda^{a} Q_{L}^{j}
\end{aligned}
$$

where $G_{\mu}^{a}$ are the gluon fields and $\lambda^{a}$ are the Gell-Mann matrices (equation A.12). The interesting pieces are given by the last two terms in equation 2.85. Hence proceeding to write them in terms of the quarks mass eigenstates:

$$
\begin{aligned}
\mathcal{L}_{\text {kinetic }}^{F C} & \supset \frac{1}{\sqrt{2}} g_{w} \bar{Q}_{L}^{j} W_{\mu}^{-} T^{-} Q_{L}^{j}+\frac{1}{\sqrt{2}} g_{w} \bar{Q}_{L}^{j} W_{\mu}^{+} T^{+} Q_{L}^{j} \\
& =\frac{1}{\sqrt{2}} g_{w}\left[\bar{u}_{L}^{j} W_{\mu}^{-} d_{L}^{j}+\bar{d}_{L}^{\prime j} W_{\mu}^{+} u_{L}^{j}\right] \\
& =\frac{1}{\sqrt{2}} g_{w}\left[\bar{u}_{L}^{j}\left(U_{L}^{\dagger} D_{L}\right)^{j k} W_{\mu}^{-} d_{L}^{k}+\bar{d}_{L}^{j}\left(D_{L}^{\dagger} U_{L}\right)^{j k} W_{\mu}^{+} u_{L}^{k}\right] \\
& =\frac{1}{\sqrt{2}} g_{w}\left[\bar{u}_{L}^{j} V^{j k} W_{\mu}^{-} d_{L}^{k}+\bar{d}_{L}^{j}\left(V^{\dagger}\right)^{j k} W_{\mu}^{+} u_{L}^{k}\right],
\end{aligned}
$$

where the new matrix $V=U_{L}^{\dagger} D_{L}$ is known as the Cabibbo-Kobayashi-Maskawa (CKM) mixing matrix. The CKM matrix is unitary $\left(V^{\dagger} V=V V^{\dagger}=\mathbb{I}\right)$ :

$$
V^{\dagger} V=\left(U_{L}^{\dagger} D_{L}\right)^{\dagger} U_{L}^{\dagger} D_{L}=D_{L}^{\dagger} U_{L} U_{L}^{\dagger} D_{L}=\mathbb{I}
$$




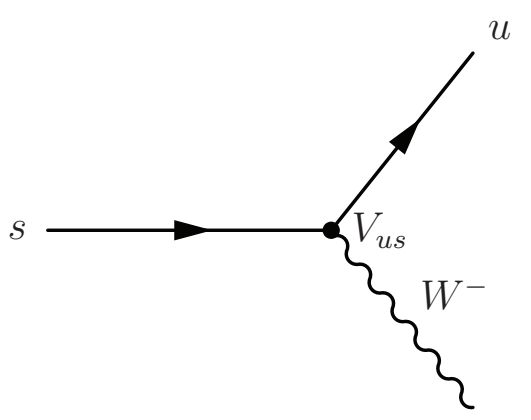

Figure 2.3: Feynman diagram depicting flavour changing interaction for strange and up quark

As an example, the flavour changing transition for the down type quark(strange quark) to the up type quark(up quark) is shown in figure 2.3.

\subsection{Overview of QCD}

The strong interaction that governs quarks and gluons is called Quantum Chromodynamics (QCD). All the quarks and gluons carries a new colour charge, which determines their interactions under QCD. This theory exhibits a strange property, known as colour confinement, which means that we can only observe colour singlet and no single colour charged particle can be directly observed. So quarks combine together to form baryons (three quarks bound state) and mesons (quark and anti-quark bound state). The strong force increases with increasing distance between quarks. Due to confinement, supplying partons (quarks and gluons ) with enough energy one can create many bound states. This processes is referred to as hadronization in collider phenomenology. In collider, these hadrons most often travel collinearly in the detector and are referred to as jets.

The strong interaction is scale dependent, which means that it varies with energy 
scales. So, the coupling of quarks and gluons decreases as the energy increases and vice versa. This behaviour is called asymptotic freedom. The variation of the strength of the strong interaction is encoded in the beta-function of the running coupling constant $\alpha_{s}[7]:$

$$
\beta(g)=\frac{d g}{d \log u} \quad, \text { where } u \text { is the energy scale }
$$

For QCD, with $n_{f}$ the number of approximately massless quarks at the energy scale and with three colours, the $\beta$ function is:

$$
\begin{gathered}
\beta(g)=-\left(11-\frac{2}{3} n_{f}\right) \frac{g^{3}}{(4 \pi)^{2}}=-\frac{b g^{3}}{(4 \pi)^{2}} \\
\text { where, } \quad b=11-\frac{2}{3} n_{f}
\end{gathered}
$$

Rewriting equation 2.88 in terms of the coupling constant $\alpha_{s}=\frac{g^{2}}{4 \pi}$ :

$$
\frac{d \alpha_{s}}{d \log u}=-\frac{b}{2 \pi} \alpha_{s}^{2}
$$

Then the solution to equation 2.90 is found to be:

$$
\alpha_{s}(u)=\frac{2 \pi}{b \log \left(\frac{u}{\Lambda}\right)}, \text { where } \Lambda \text { is the integration constant }
$$

It clear from equation 2.91 that the coupling strength decreases for an increasing $u$ and perturbation fails when $u=\Lambda$ which is referred to as the $Q C D$ scale $\Lambda_{Q C D}$ $\left(\Lambda_{Q C D} \approx 200 \mathrm{MeV}\right)$. 


\section{Chapter 3}

\section{Our Model and Motivation}

Before we begin to explore our model and its motivation, lets focus on how one would calculate some quantities of interest in the model. In general, particles are not stable so they will decay to other particles after a certain amount of time. Given a model, it is possible to compute the lifetime of a particle. In addition, in experiments one most often collides a known set of particles to explore the substructure of the particle of interest or produce new exotic and unknown particles. To that end, another quantity called the cross section is very important, which is related to the probability of an occurrence of a particular process of interest. In order to study various processes, one needs to be able to describe and quantify them precisely. A brief overview of calculating decay widths and cross sections can be found in appendix B.5 and B.6.

\subsection{Motivation}

In light of its successes, the SM cannot be the complete description of the universe. As one notices right away, the SM makes no mention of gravity, which is the oldest known force of nature and is extremely important at large scales. In addition, there is a lack of explanation for dark matter. Now with the discovery of the Higgs boson, which is 
responsible for electroweak symmetry breaking, one is faced with the problem of the quadratic divergence of the Higgs mass term from loop contributions. The dominant loop contribution to the Higgs mass term comes from a top quark loop since the top quark couples most strongly to the Higgs field. This can be seen from the Yukawa interaction Lagrangian for fermions, where the Yukawa coupling is proportional to the the mass of the fermion $\left(y_{t} \propto \frac{m_{t}}{v}\right)$.

The one loop correction from the $\left(\phi^{*} \phi\right)^{2}$ term of the self-energy diagram to $\phi^{*} \phi$ term in the Higgs potential is of the form:

$$
\delta\left(\phi^{*} \phi\right) \propto \int^{\Lambda} \lambda \frac{d^{4} k}{(2 \pi)^{4}} \frac{1}{k^{2}-m_{h}^{2}} \approx \lambda \frac{\Lambda^{2}}{16 \pi^{2}} \phi^{*} \phi
$$

where $\Lambda$ is the scale for the new physics to manifest itself. The one loop contribution from the top quark has a similar form. The one loop contribution to the Higgs mass from the top quark give rise to the following relation:

$$
\mu_{\mathrm{phys}}^{2} \simeq \mu^{2}-\frac{1}{16 \pi^{2}} y_{t}^{2} \Lambda^{2}
$$

where $\mu$ is a parameter in the quadratic term of the Higgs potential and $y_{t}$ is the top Yukawa coupling. From equation 2.35, we have $\mu_{\text {phys }}^{2}=\lambda v^{2}$ that determines the weak scale. Furthermore equation 2.39 relates the Higgs mass, which is experimentally established, to $\mu_{\text {phys }}$ as:

$$
m_{h}=\sqrt{2} \mu_{\text {phys }}
$$

In order to obtain the observed Higgs mass, the new physics scale must lie close to the weak scale; otherwise, $\mu$ needs to be fine-tuned. So far there has not been any evidence of new particles near the weak scale $(v \approx 246 \mathrm{GeV})$ so it appears that 
the SM requires some fine-tuning and the problem becomes worse as the scale $\Lambda$ approaches the Planck scale. In the SM, $\mu_{\text {phys }}$ not only affects the Higgs mass but all the particles that get their masses through the Higgs mechanism. The masses of the $\mathrm{W}$ and $\mathrm{Z}$ bosons(shown in equations 2.56 and 2.53 ) are directly related to $\mu_{\mathrm{phy}}$ :

$$
m_{W}=\frac{v g_{w}}{2}=\frac{g_{w} \mu_{\mathrm{phys}}}{2 \sqrt{\lambda}} \quad, m_{Z}=\frac{\sqrt{g^{2}+g_{w}^{2}} v}{2}=\frac{\sqrt{g^{2}+g_{w}^{2}} \mu_{\mathrm{phys}}}{2 \sqrt{\lambda}}
$$

Thus changing $\mu_{\text {phys }}$ affects all the SM masses.

Now one might be curious as to why are we only concerned with the Higgs boson? Does the divergence also appear in other sectors? The answer is no! The quadratic divergence appears only in the Higgs boson because it is the only scalar in the Standard Model. Unlike fermions whose masses are protected by chiral symmetry or the gauge bosons whose masses are protected by the gauge symmetry, the Higgs boson is not protected by any symmetry against divergences. So we run into the problem of extreme fine-tuning as the energy scale $\Lambda$ approaches $\Lambda_{\text {Planck }}$.

Now how does one go about solving the fine-tuning problem? The most notable possibility is supersymmetry(SUSY), which relates bosons and fermions. SUSY doubles the number of particles in the SM by proposing that each of the SM particle has a partner (referred to as superpartner) with a spin that is different by a half integer. However, the lack of experimental evidence and with the LHC imposing strong constraints on most of the superpartners suggest that perhaps we need to look at an alternative solutions. One such non-susy approach involves the introduction of a new fermionic partner called the top partner and we are interested in such an approach. As a matter of fact, top partners have been studied at colliders and they involve coupling to the third generation quarks. The generic top partner decay topologies studied at the LHC are: $T \longrightarrow t h, T \longrightarrow t Z, T \longrightarrow b W$ as shown in figure 3.1. But 
there are strong constraints on these topologies and the typical lower mass limit on the top partner is around $m_{T} \geq 800 \mathrm{GeV}[5,6]$. Instead of the above decay modes, we consider a scenario where the top partner can decay into some other particles leading to new signal topologies.
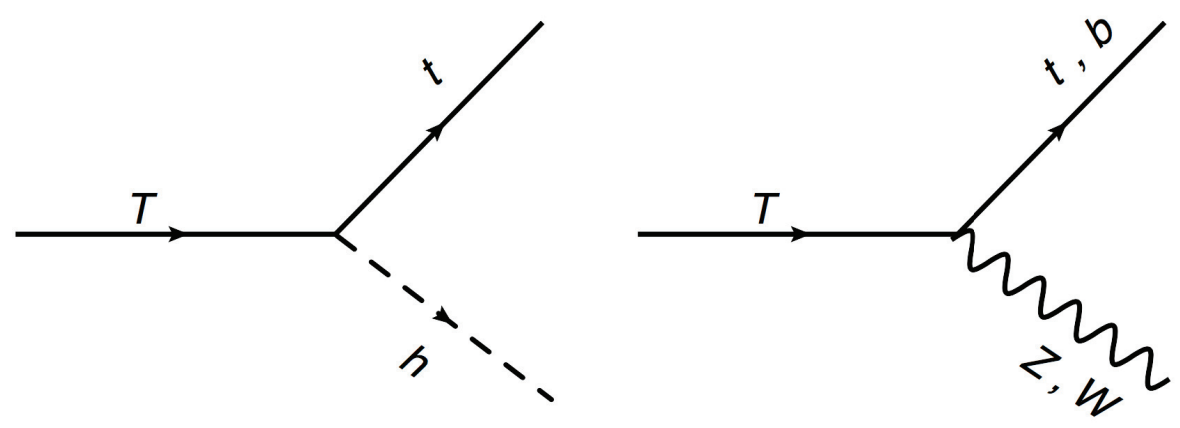

Figure 3.1: The typical top partner decay channels investigated at the LHC.

\subsection{Our Model}

We are interested in an extension of the fermionic sector of the SM, which contains a new fermionic top partner $(T)$ that has the same quantum number as the SM top quark. The top partner is a colour triplet (it transforms in the fundamental representation of $\left.S U(3)_{c}\right)$, which means that it behaves in the same way as the SM quarks under the strong force. Moreover, $T$ has an electric charge of $Q=+\frac{2}{3} e$ and it is not charged under the $\mathrm{SM} S U(2)_{L}$ gauge group (T is an $S U(2)_{L}$ singlet). This fermion has a Yukawa type interaction with the Standard model quarks. The top partner is a vector-like fermion which means that it's left and right handed components have the same transformation under the SM symmetry group $\left(S U(3)_{c} \times S U(2)_{L} \times U(1)_{Y}\right)$. Consequently, the top partner can get a mass independent of its coupling to the Higgs field. 
The are many possible decay channels for the top partner. We have mentioned some of the generic decay modes in section 3.1. However, these are strongly constrained by the experimental searches. So we want to look at alternative decays that can relax the constraints on the top partner from the current experimental searches, hence explain how it could have escaped the detection so far. The model that we explore has two main decay channels. First, we investigate the top partner decay primarily to three light quarks $(u$ and $c)$. Second, the top partner decay to a top quark and two light quarks $(u$ and $c)$. We consider the interaction to the rest of the SM quarks to be extremely suppressed. The decay proceeds in two steps: first the top partner decays to an up-type quark and a new scalar $(\eta)$, then this scalar decays to a quark and an anti-quark. In the Weyl basis the top partner field can be expressed as:

$$
T=\left(\begin{array}{c}
T_{L} \\
T_{R}
\end{array}\right), \quad \text { where } T_{L}=\frac{1-\gamma^{5}}{2} T, T_{R}=\frac{1+\gamma^{5}}{2} T
$$

The Lagrangian for our model is given as:

$$
\begin{aligned}
& \mathcal{L}=i \bar{T} \not D T+\frac{1}{2} \partial_{\mu} \eta \partial^{\mu} \eta-m_{T} \bar{T} T-\frac{1}{2} m_{\eta} \eta \eta-\mathcal{L}_{i n t} \\
& \text { where } D_{\mu}=\partial_{\mu}-i\left(g_{w} c_{W} T^{3}-g s_{W} Y\right) Z_{\mu}-i g_{s} G_{\mu}^{a} T_{a}
\end{aligned}
$$

The scalar field $\eta$ is not charged under $S U(2)_{L}$. The interaction Lagrangian $\mathcal{L}_{\text {int }}$ for the two cases is: 
1. Coupling to the SM light quarks:

$$
\begin{gathered}
\mathcal{L}_{\text {int }}=\lambda \eta \bar{c} P_{L} T+\epsilon \eta \bar{u} P_{L} u+\text { h.c. } \\
\text { where, } P_{L}=\frac{1}{2}\left(1-\gamma^{5}\right)
\end{gathered}
$$

where $\lambda$ and $\epsilon$ are the coupling constants. The $P_{L}$ is there to make the term gauge invariant.

2. Coupling to a heavy quark (t) and two light quarks:

$$
\mathcal{L}_{i n t}=\zeta \eta \bar{t} P_{L} T+\epsilon \eta \bar{u} P_{L} u+\text { h.c. }
$$

where $\zeta$ is a coupling constant.

The Feynman rules for this model are given as,

1. Propagators:

$$
\begin{aligned}
& \grave{\eta(x) \eta}(y)=---\overline{\mathrm{q}}---=\frac{i}{q^{2}-m_{\eta}^{2}+i \epsilon} \\
& \overrightarrow{T(x) \bar{T}}(y)=\underset{\mathrm{q}}{\longrightarrow}=\frac{i\left(q+m_{T}\right)}{q^{2}-m_{T}^{2}+i \epsilon}
\end{aligned}
$$

2. Vertices:

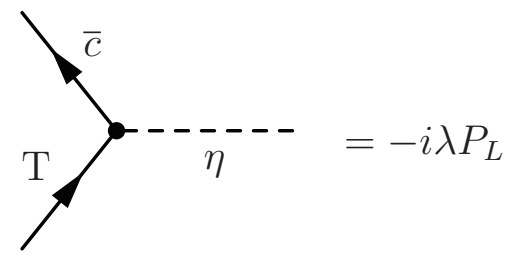



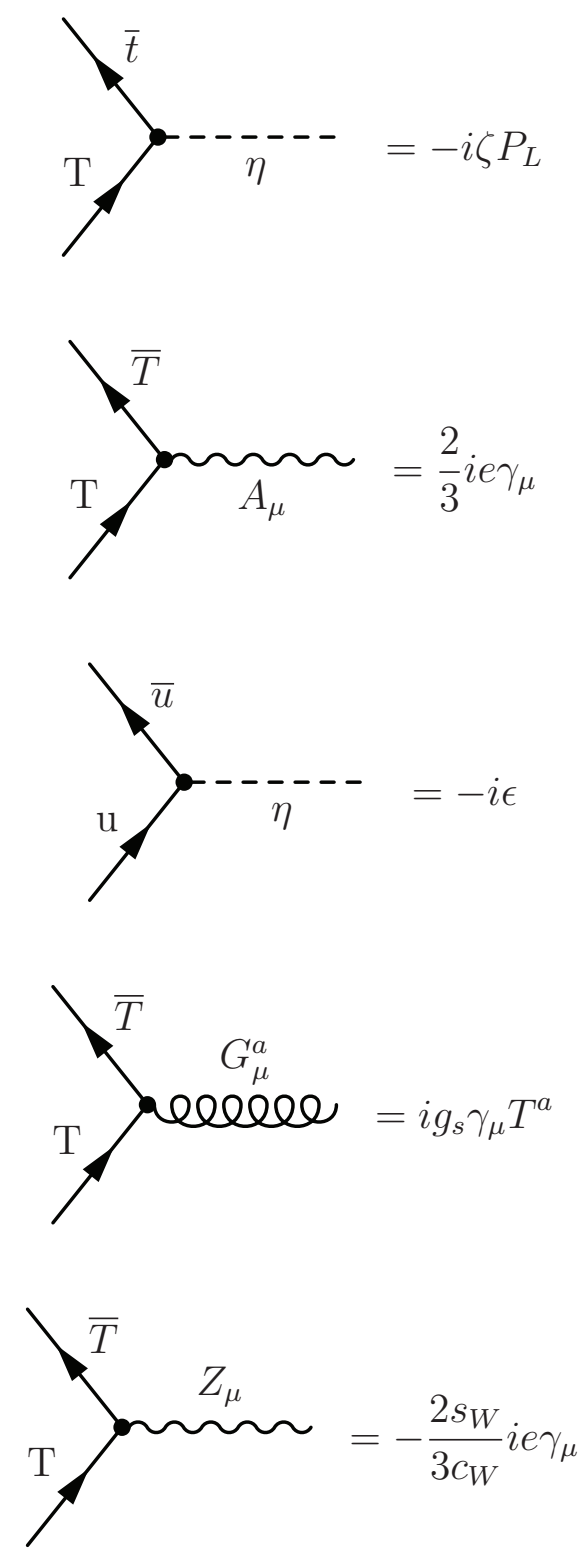

3. External leg contractions:

$$
\overrightarrow{\eta(x) \mid \mathbf{q}}\rangle=>\mathbf{q}---1 \quad\langle\mathbf{q}| \eta(x)=----<=1
$$



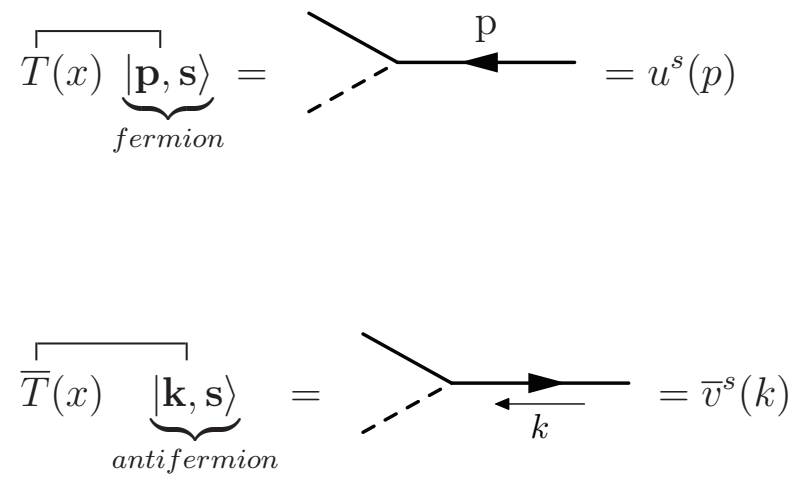
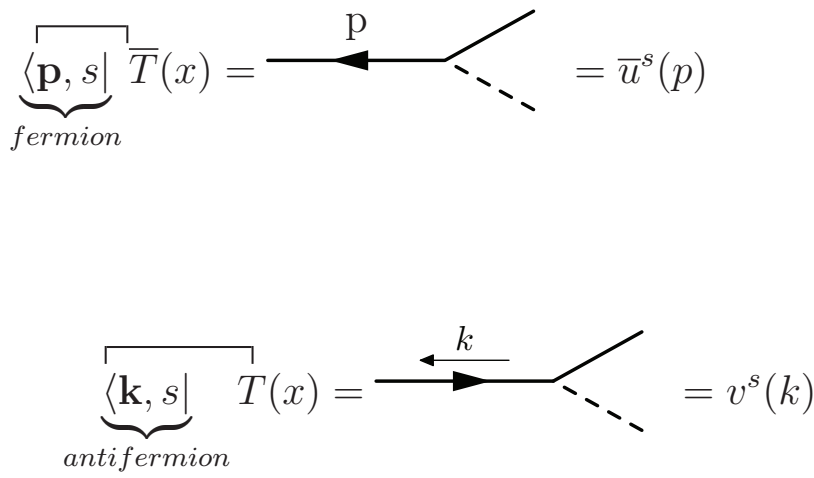

4. At each vertex, we impose four-momentum conservation

5. Complete the integration over each of the undetermined loop momenta

6. Determine the overall sign of the diagram

With the Feynman rules in hand, one possesses the ability to calculate the decay width for the top partner and the scalar field $\eta$. The Feynman diagram for the 2-body top partner decay is:

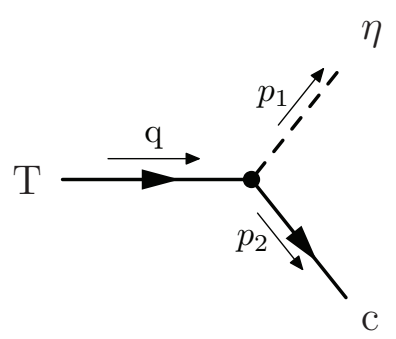

The matrix element for the above process is given as:

$$
i \mathcal{M}=i \lambda \bar{u}\left(p_{2}\right) P_{L} u(q), \quad(i \mathcal{M})^{\dagger}=-i(\lambda)^{*} \bar{u}(q) P_{R} u\left(p_{2}\right)
$$

In the second expression $P_{L} \longrightarrow P_{R}$ because $\gamma^{5}$ anti-commutes with $\gamma^{0}\left(\left\{\gamma^{0}, \gamma^{5}\right\}=0\right)$. 
Averaging over the initial and summing over the final spins:

$$
\begin{aligned}
& \frac{1}{2} \sum|\mathcal{M}|^{2}=\frac{1}{2} \sum\left[-i \lambda^{*} \bar{u}(q) P_{R} u\left(p_{2}\right)\right]\left[i \lambda \bar{u}\left(p_{2}\right) P_{L} u(q)\right] \\
& =\frac{\lambda^{2}}{2} \sum \bar{u}(q) P_{R} u\left(p_{2}\right) \bar{u}\left(p_{2}\right) P_{L} u(q) \\
& =\frac{\lambda^{2}}{2} \sum \bar{u}(q) u\left(p_{2}\right) \bar{u}\left(p_{2}\right) u(q) P_{L}, \quad \text { passing } P_{R} \text { through becomes } P_{L} \\
& =\frac{\lambda^{2}}{2} \operatorname{tr}\left[\left(\not q+m_{T}\right)\left(\not p_{2}+m_{c}\right) P_{L}\right]
\end{aligned}
$$

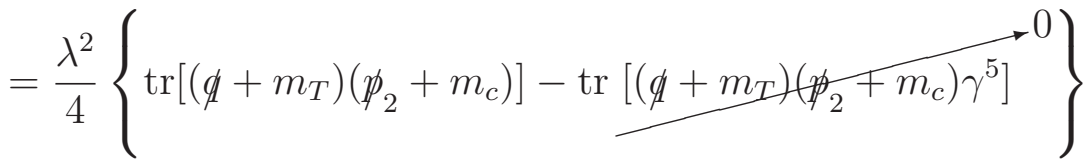

$$
\begin{aligned}
& =\frac{\lambda^{2}}{4}\left\{\operatorname{tr}\left[\not p_{2}\right]+\operatorname{tr}\left[m_{T} m_{c}\right]\right\}=\lambda^{2}\left[q \cdot p_{2}+m_{T} m_{c}\right]
\end{aligned}
$$

The second term in the second line is zero since the trace of the $\gamma^{5}$ matrices vanishes (appendix B.2). Doing the kinematics in the centre of mass (COM) frame:

$$
\begin{aligned}
q=\left(m_{T}, \overrightarrow{0}\right), \quad p_{1} & =\left(E_{\eta}, \vec{p}_{1}\right), \quad p_{2}=\left(E_{c},-\vec{p}_{1}\right), \text { where } E_{\eta, c}=\sqrt{m_{\eta, c}^{2}+p_{1}^{2}} \\
q=p_{1}+p_{2} \longrightarrow p_{1}^{2} & =\left(q-p_{2}\right)^{2}=q^{2}+p_{2}^{2}-2 q \cdot p_{2} \\
m_{\eta}^{2} & =m_{T}^{2}+m_{c}^{2}-2 m_{T} E_{c} \Longrightarrow E_{c}=\frac{m_{T}^{2}+m_{c}^{2}-m_{\eta}^{2}}{2 m_{T}} \\
\text { Then }\left|\overrightarrow{p_{1}}\right| & =\sqrt{\frac{m_{T}^{2}+m_{c}^{2}-m_{\eta}^{2}}{2 m_{T}}-m_{c}^{2}}, \quad q \cdot p_{2}=m_{T} E_{c}
\end{aligned}
$$

The expression for the decay width is (equation B.36):

$$
\begin{aligned}
& \Gamma=\frac{|\mathcal{M}|^{2}}{2 m_{T}} \int \mathrm{d} \Pi_{n} \quad \text { Here } \mathrm{n}=2 \text { (number of particles in the final state) } \\
& \text { where } \int \mathrm{d} \Pi_{n} \equiv\left(\prod_{f} \int \frac{\mathrm{d}^{3} p_{f}}{(2 \pi)^{3} 2 E_{f}}\right)(2 \pi)^{4} \delta^{4}\left(P-\sum P_{f}\right)
\end{aligned}
$$

The quantity $\int d \Pi_{n}$ is known as the relativistically invariant $n$-body phase space. 
Using the expression $\int d \Pi_{2}=\int \frac{\|\vec{p}\| d \Omega}{16 \pi^{2} m}$ (calculated in equation B.40), the top partner width becomes:

$$
\begin{aligned}
\Gamma_{T} & =\frac{1}{2 m_{T}} \frac{\left\|\overrightarrow{p_{1}}\right\|}{16 \pi^{2} m_{T}}(4 \pi) \lambda^{2}\left(q \cdot p_{2}+m_{T} m_{c}\right) \\
& =\frac{\lambda^{2}}{8 \pi m_{T}^{2}}\left(E_{c}^{2}-m_{c}^{2}\right)^{\frac{1}{2}}\left(m_{T} E_{c}+m_{T} m_{c}\right) \\
& =\frac{\lambda^{2}}{8 \pi m_{T}}\left(E_{c}+m_{c}\right)^{\frac{3}{2}}\left(E_{c}-m_{c}\right)^{\frac{1}{2}} \\
& =\frac{\lambda^{2}}{32 \pi m_{T}^{3}}\left(m_{T}^{2}+m_{c}^{2}-m_{\eta}^{2}+2 m_{T} m_{c}\right)^{\frac{3}{2}}\left(m_{T}^{2}+m_{c}^{2}-m_{\eta}^{2}-2 m_{T} m_{c}\right)^{\frac{1}{2}}
\end{aligned}
$$

In the limit $m_{c} \longrightarrow 0\left(E \gg m_{c}\right)$, then $E_{c} \approx \frac{m_{T}^{2}-m_{\eta}^{2}}{2 m_{T}}$. Thus the top partner decay width becomes:

$$
\Gamma_{T}=\frac{\lambda^{2}}{32 \pi m_{T}^{3}}\left(m_{T}^{2}-m_{\eta}^{2}\right)^{2}
$$

Note for the case where $m_{\eta}$ is heavier than the top partner, then the top partner decays as $T \longrightarrow c \bar{u} u$ and the width of the top partner is calculated using MadGraph5_aMC@NLO [10]. Similarly, the width of the scalar $\eta$ has been calculated to be (appendix B.7):

$$
\Gamma_{\eta}=\frac{3 \epsilon^{2} m_{\eta}}{8 \pi}\left(1-\frac{4 m_{u}^{2}}{m_{\eta}^{2}}\right)^{\frac{3}{2}}
$$

\subsection{Our Model Phenomenology}

The exciting thing about this model is its simplicity accompanied with the constraint only imposed on $m_{T}$ and $m_{\eta}$. We are going to investigate all the points in the $m_{T} m_{\eta^{-}}$ plane and determine the allowed parameter space. The top partner is expected to be within the reach of the LHC. So lets begin by exploring it's possible collider signatures 
for each of the cases under study.

\subsubsection{Case 1: Top Partner Decay to Light Quarks}

The model has a fully hadronic signature with many jets in the final state as can be seen from the interaction term in the Lagrangian (equation 3.7). Coupling $T$ to only the light quarks will results exclusively in jets, which are a collection of collimated hadrons resulting from hadronization of quarks and gluons. To study the phenomenology of this model, we need to explore its jet activity. The Feynman diagram for the production and decay of the top partner is shown in figure 3.2. The main signatures would be multiple jets. For example, in the Large Hadron Collider (LHC) at CERN, the proton-proton collision will lead to the pair production of top partners and the events will include at least 6 -jets. There is a resonance structure among the six jets so we can reconstruct the top partner from three jets in the final state.

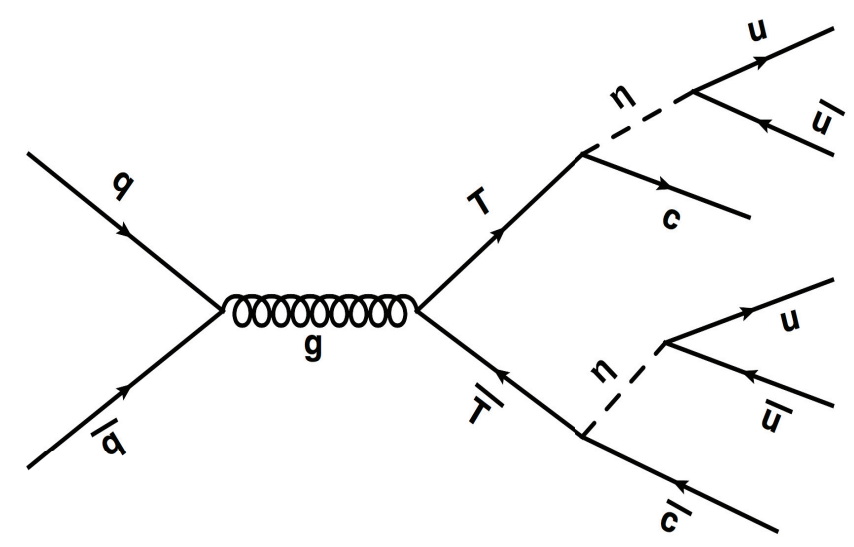

Figure 3.2: Feynman diagram for the top partner decay to light quarks. 


\subsubsection{Case 2: Top Partner Decay to a Heavy Flavour Quark}

For this particular scenario the final state signatures are dependent upon the various decay products of the top quark. As shown in figure 3.3, the final state contains $W$ 's so it can either result in fully hadronic or hadronic plus leptonic signal topology. There are three possible cases for the observed events: jets +0 leptons, jets +1 lepton, jets +2 leptons. The branching fraction for the W's are given as [11]:

$$
\begin{aligned}
& \operatorname{Br}(W \longrightarrow q \bar{q})=0.6741 \pm 0.0027 \\
& \operatorname{Br}\left(W \longrightarrow l \nu_{l}\right)=0.1086 \pm 0.0009
\end{aligned}
$$

where $\mathrm{q}(\bar{q})$ are quarks (anti-quarks), $l$ are the charge leptons and $\nu_{l}$ are the neutrinos.

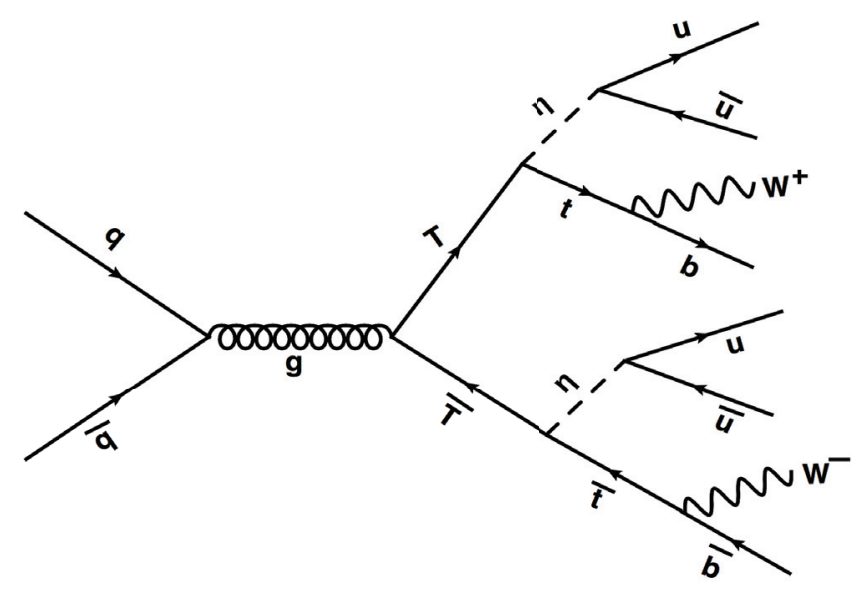

Figure 3.3: Feynman diagram for the top partner decay to a heavy and two light quarks.

In addition, the top quark decays as $t \longrightarrow b W$ nearly $100 \%$ of the time, which can be seen from the CKM matrix elements $\left(\left|V_{t b}\right|=0.99915 \pm 0.00005\right.$ and it is much bigger than all the other $\left|V_{t q}\right|$ 's). So the signal topology always contains $b$-quarks and leptons, which assist in better discriminating the signal from the background by employing 
b-tagging(identifying jets associated with b-quarks) and lepton identification. The background is reduced significantly due to identifying hard leptons so it is expected that the search will result in constraints that are somewhat more stringent than the light flavour scenario. 


\section{Chapter 4}

\section{Analysis and Strategies}

As we have previously mentioned, we are interested in two main cases. First, the model with fully hadronic decay has multi-jet final states in the detector, which narrows down the possible searches that can be sensitive to this model. Secondly, the model with the top partner coupling to the top quark can give rise to both hadronic and lepton signatures. However, most of the searches focused on studying beyond the Standard Model at the LHC use simplified supersymmetric models as a benchmark. The majority of them have a large missing energy or missing transverse momentum as part of their requirements. However, these are not of particular interest to us because the light flavour and heavy the flavour scenario (fully hadronic final state) involves no missing energy. For our heavy flavour scenario, we could get some missing energy in the final state due to the presence of neutrinos(if the W-boson decay leptonically). Consequently they are insensitive to our signal topologies. So the appropriate searches to consider are the ones with only jets or jets and leptons with little to no missing energy in the final state. The simplified supersymmetric models that exhibit such behaviour at colliders are R-parity violating (RPV) models. R-parity is 
a $\mathbb{Z}_{2}$ symmetry that is defined as:

$$
R=(-1)^{3(B-L)+2 s}, \quad \text { where } s=\text { Spin }, B=\text { Baryon number }, L=\text { Lepton number }
$$

All the supersymmetric particles have R-parity of -1, while all the SM particles have $\mathrm{R}$-parity of +1 . So one sees that for R-parity conserving models, the supersymmetric model must contain a stable lightest supersymmetric particle (LSP) and the superpartners must be produced in pairs. Consequently, the LSP escapes the detector and it inevitably leads to missing energy (if the LSP is neutral). On the other hand in RPV models, the LSP can decay to SM particles which can result in jets and no missing energy in the final states. Thus the resulting signal events have a similar topology to our model. For example, a topology of the form shown in figure 4.1 is possible in a RPV model and it has been looked for at the LHC [12-14]. Now that we have our model and identified the possible LHC searches that could be sensitive to it, how do we move forward with putting constraints on the model parameter space?

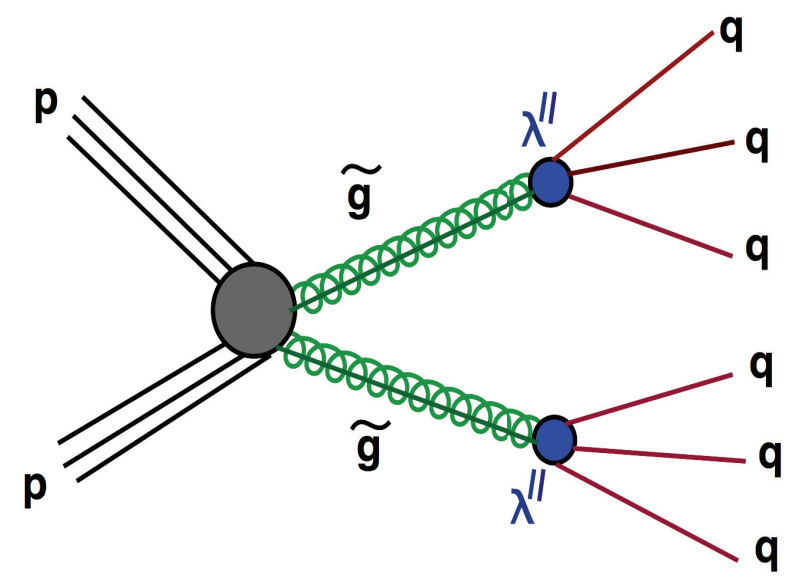

Figure 4.1: A schematic diagram of a typical multi-jet RPV topology where gluinos are pair produced and each then decays to the SM quarks. 
In a collider such as the LHC, proton-proton collisions produce lots of soft partons and a small fraction of collisions lead to high momentum transfer events (hard scatter). For the hard scatter processes, the Parton Distribution Functions (PDFs) describe partons (gluons and quarks) coming into the process and perturbation theory gives the probability distribution of the outgoing partons [15]. Since partons themselves carry colour, they themselves can radiate gluons and quarks resulting in an extended shower. This is the parton shower phase of the process which stops when the radiated partons have momentum of the order of the QCD scale.

Because the partons can't be observed isolated due to colour confinement they form hadrons and this is what is referred to as hadronization. The Matrix Element (ME) description of partons diverges as the partons become soft and collinear. So we need to resort to another procedure that must take over in that limit to achieve an accurate representation of the QCD processes. The Parton Shower (PS) description is accurate when the partons are soft and collinear but unreliable if the partons are widely separated and hard. So for a QCD process to be effectively described in a Monte Carlo event generator, one needs to consider the ME and PS representations in combination.

\subsection{Implementation Tools}

In order to implement our model and study its phenomenology, we take advantage of several publicly available software packages. First, FeynRules which is a MATHEMATICA based package that can be used to calculate Feynman rules for any physics model [16]. The package requires the quantum fields information, the parameters of the model and the Lagrangian. At the end, FeynRules returns an output appropriate for various Monte Carlo event generation programs. Thus FeynRules 
provides the first step in building a new theoretical model and paves the way toward comparing a theory of interest with experimental observations. Once we have the model, our primary goal is to establish constraints on the model which requires simulating collider events.

One powerful tool capable of simulating events according to a specified model is MadGraph5_aMC@NLO, which is a Monte Carlo event generator that creates Feynman diagrams for the desired process [10]. MadGraph5_aMC@NLO is also capable of computing tree-level and next-to-leading order cross sections and their matching to parton shower simulations. Next, the events are passed to PYTHIA8, which is an event showering and hadronization program [17]. After the shower and hadronization, the resulting events are then processed by DELPHES, which is a detector simulator that recreates physics objects $\left(e^{-}, \mu^{-}\right.$, jets, etc ...) from a simulated detector response [18]. So the output is Monte Carlo events that can be analyzed to give physicists an idea of what to expect at colliders and compare the results to the current observations. A pictorial representation of the process is given in the schematic diagram in figure 4.2 .

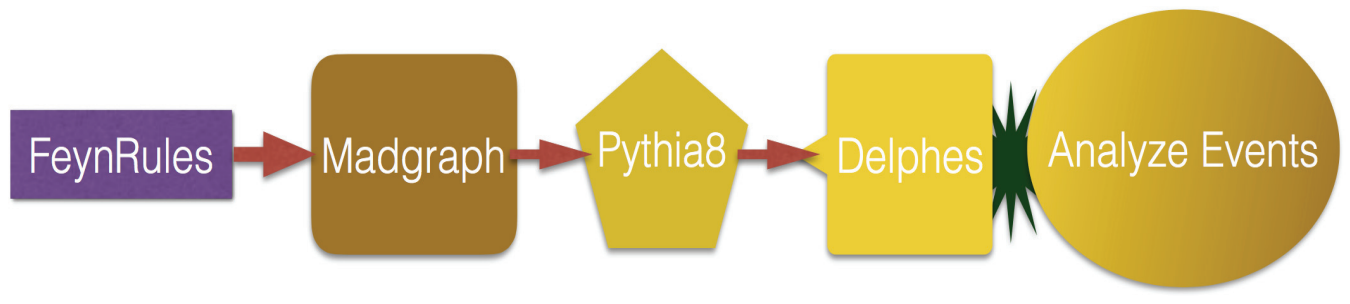

Figure 4.2: A schematic diagram shows the various steps and the programs used in simulating events for our model. 


\subsection{QCD Multi-jet Background}

Now in order to investigate such models, one needs to be aware of the dominant Standard Model background associated with it. In a collider, the initial and final state quarks can radiate additional partons (initial state radiation and final state radiation respectively) and the partons inside the hadrons can interact resulting in multiparton interactions. Moreover, multi-jet events can contain large missing energy through jet mismeasurements and the neutrinos coming from the decay of heavy flavour quarks. Thus the SM background from events containing multiple jets can become significant at high energies due to the strong dynamics of QCD.

The dominant background for our model comes from multi-jet events. As an illustration, we show in figure 4.3 the differential cross section as a function of the leading jet transverse momentum. Also for comparison, in figure 4.4 we have plotted the production cross section for the top partner $(\sigma(p p \longrightarrow \bar{T} T))$ which was computed using MadGraph5_aMC@NLO. Notice that the cross section for the multi-jet is $\approx 10^{5} \mathrm{pb}$ for the leading jet transverse momentum of $100 \mathrm{GeV}$. On the other hand, the production cross section for the top partner is $\approx 10^{2}-10^{-2} \mathrm{pb}$. Hence, the background contribution from multi-jet events is significant. So clever strategies are required in order to reduce the background and observe the signal events for the desired model. Typically the contribution from a QCD process at high energy is calculated using perturbative QCD(expansion in the strong coupling constant). But this method works well only if the number of particles is small and it becomes very complicated as the number of particles increases. Hence this approach is not ideal for the LHC and the QCD background for physics beyond the SM are estimated from data. 


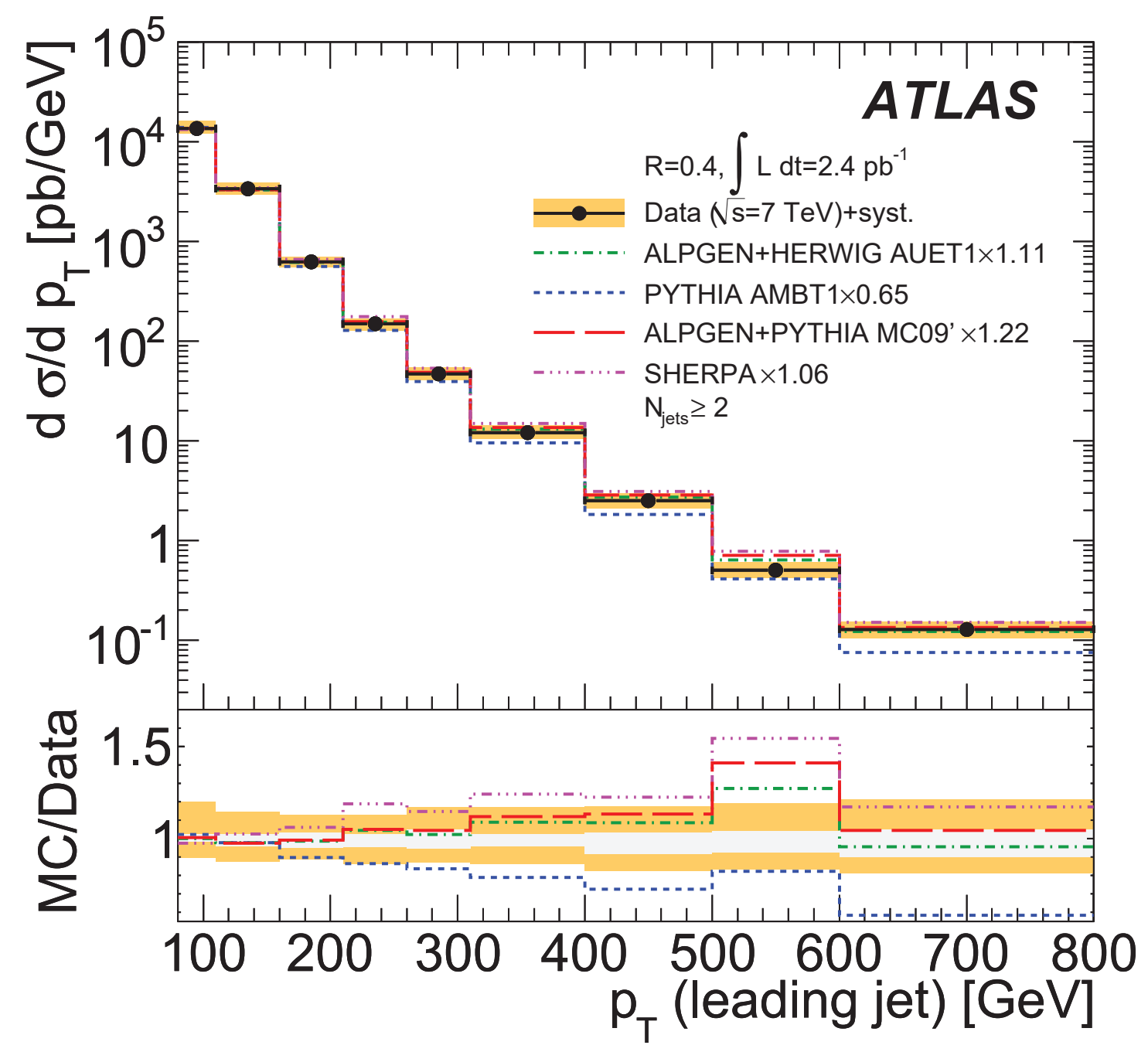

Figure 4.3: Differential Cross section as a function of the leading jet transverse momentum at $\sqrt{s}=7 \mathrm{TeV}$ (center of mass energy) with $N_{\text {jet }} \geq 2$ [19].

\subsection{Matching and Merging}

As mentioned previously, the Matrix Element (ME) description of partons is unreliable as the partons become soft and collinear and the Parton Shower (PS) description is accurate when the partons are soft and collinear but unreliable if the partons are widely separated and hard. So we need to consider ME and PS representation in 


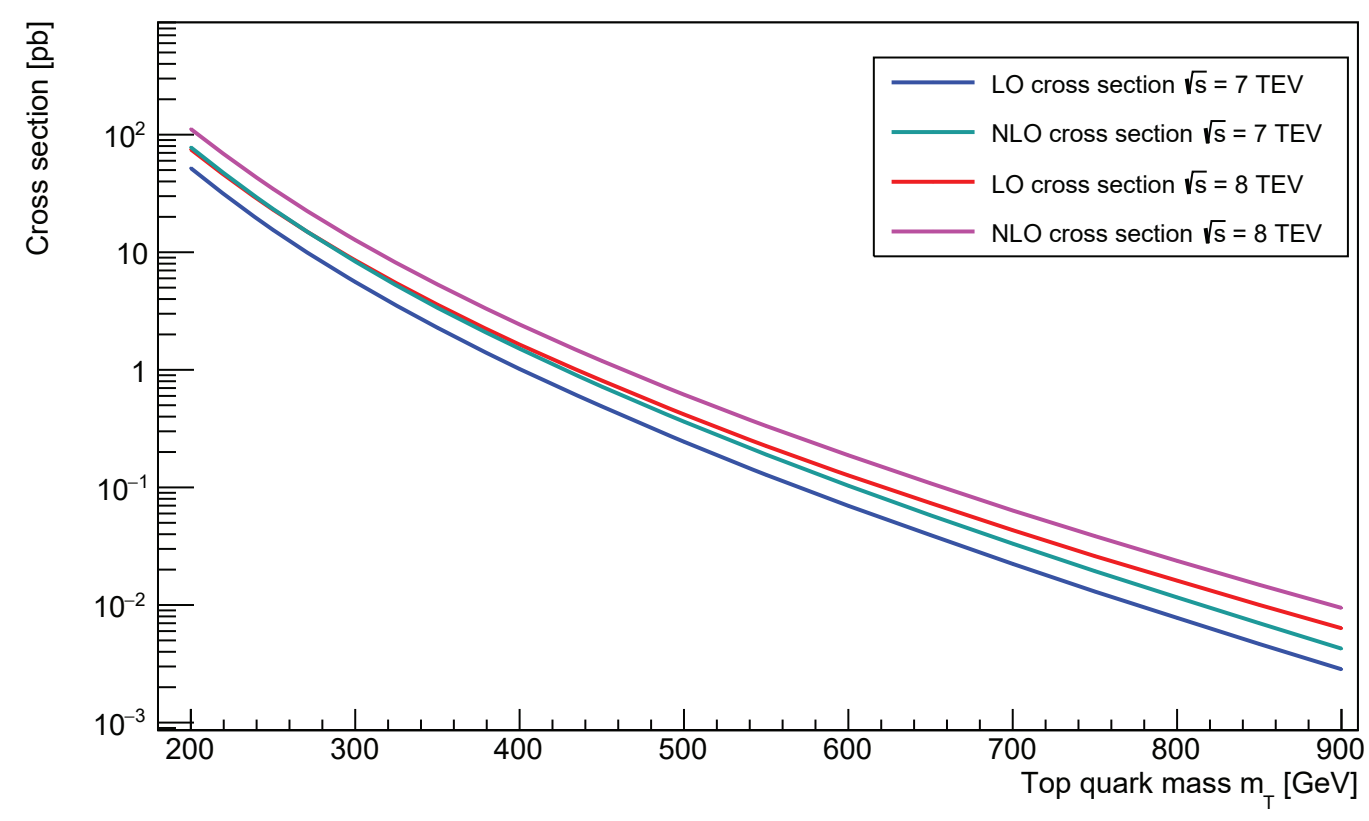

Figure 4.4: The top partner production cross section as a function of the top partner mass at $\sqrt{s}=7,8 \mathrm{TeV}$ at leading and next to leading order.

combination in order to accurately describe partons and that is where the parton jet matching comes to the rescue. To achieve this, one needs to generate events in MadGraph5_aMC@NLO and shower them using Pythia but keep in mind that without further processing, this will lead to double counting. The goal of the jet matching [20] procedure is to avoid this double counting in the overlapping regions of phase space.

In the implementation stage, we need to tell MadGraph5_aMC@NLO not to generate events that are too soft or collinear since MadGraph5_aMC@NLO utilizes ME to perform event generations which is not accurate in the regime of soft and collinear partons. After MadGraph5_aMC@NLO, the events are passed into Pythia for showering and after showering but before hadronization, the final state partons are clustered into jets with a particular jet clustering algorithm [20]. The jet clustering 
algorithms are methods used to approximately reconstruct the process of hadronization and fragmentation. There are many jet algorithms available but here we will just briefly outline the anti- $k_{t}$ jet clustering algorithm [21]. The anti-kt algorithm relies on the distance measurements defined as follows:

$$
\begin{aligned}
d_{i j} & =\min \left(k_{T_{i}}^{2 p}, k_{T_{j}}^{2 p}\right) \frac{\Delta_{i j}}{R}, \quad \text { where } \Delta_{i j}=\sqrt{\left(\phi_{i}-\phi_{j}\right)^{2}+\left(y_{i}-y_{j}\right)^{2}} \\
d_{i B} & =k_{T}^{2 p}
\end{aligned}
$$

where $d_{i j}$ is the distance between two particles $i$ and $j, R$ is the radius of the jet, $k_{T}$ is the transverse momentum, and $d_{i B}$ is the distance of particle $i$ and the beam. The parameter $p$ governs the relative power of the energy vs the geometrical $\left(\Delta_{i j}\right)$ scales and $p=-1$ for the anti- $k_{t}$ algorithm. First, the algorithm computes all the distances $\left(d_{i j}\right.$ and $\left.d_{i B}\right)$ and find the smallest. If $d_{i j}$ is the smallest, combine particle $i$ and $j$ and proceed to finding the next smallest distance. If $d_{i B}$ is the smallest, remove particle $i$ and call it a jet. Repeat the procedure until all the available particles are clustered into jets.

The matching algorithms are very involved but the basic idea is that below a certain cut off, the jet distribution is given by PS and above the cut off is described by ME. Now why are we concerned with matching? Matching is necessary for large number of high $p_{T}$ jets since Pythia doesn't give accurate results in the high $p_{T}$ regime. For illustration, we have plotted the cross section for an ATLAS analysis, which will be discussed in more details later in chapter 5 , as a function of the top partner mass in figure 4.5 with matching and without matching. So it is an important effect for our results as can be seen in figure 4.5. The figure shows the visible cross section for $m_{\eta}=200 \mathrm{GeV}$ as a function of the top partner mass using matching and without matching. 


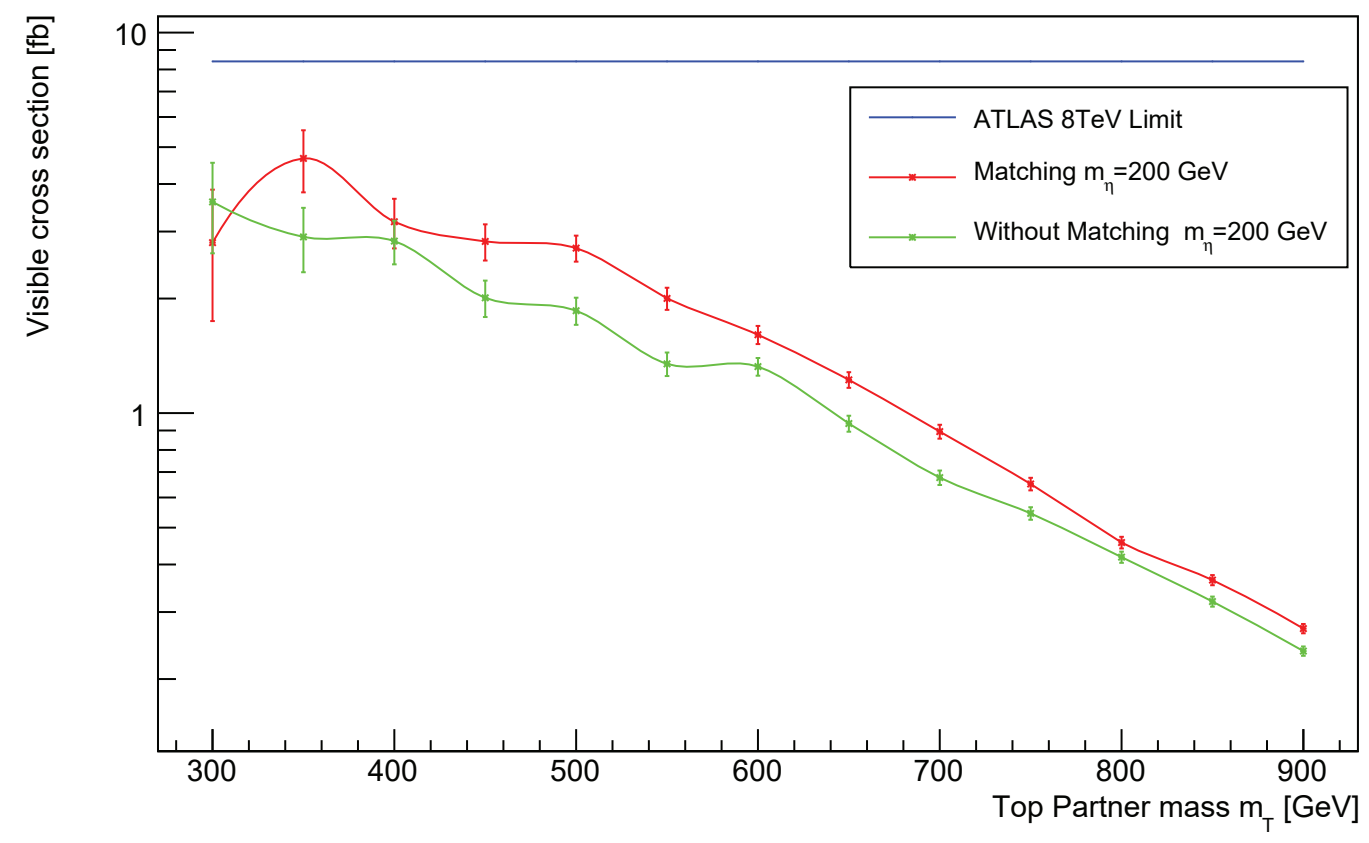

Figure 4.5: The result of an ATLAS search cross section for $m_{\eta}=200 \mathrm{GeV}$ with matching and without matching.

\subsection{Pythia6 VS Pythia8}

There are two main versions of Pythia that are currently available, Pythia6 [22] (older version)and Pythia8 (latest version) [17]. Over the course of time we have used both to see if they produce similar results. Testing both of the showering/hadronization programs, it was found that there is some discrepancy in the underlying event production. As an illustration, we have have plotted a cross section as function of the top partner mass for an ATLAS search (described in chapter5) for various $m_{\eta}$ in figure 4.6 . 


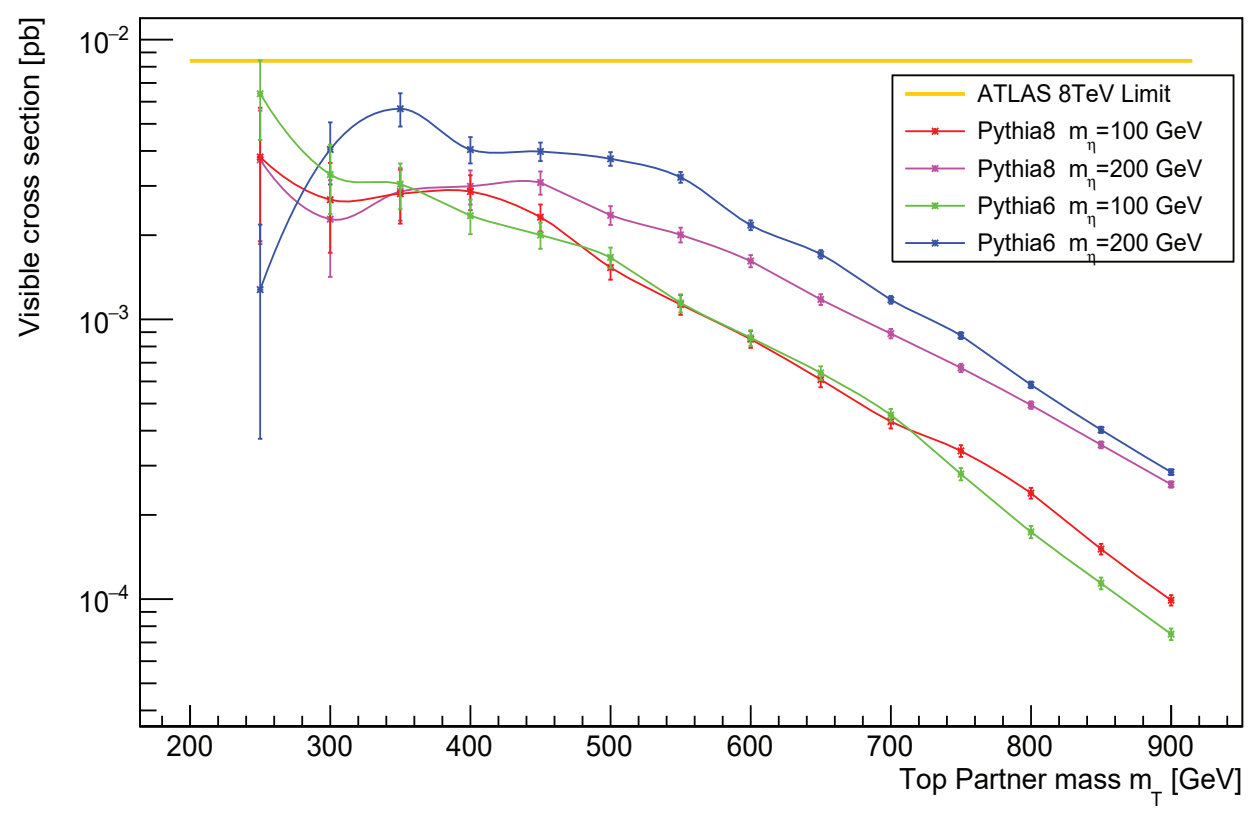

Figure 4.6: The figure shows the cross section for an ATLAS search using Pythia8 and Pythia6 for various $\eta$ masses.

The important thing to notice is that there is slight discrepancy between the two versions of Pythia. In order to understand the underlying reason, we have changed some of the underlying event production in the two Pythia's version and compared the final result for any significant differences. The underlying event generation in Pythia is controlled by parameters referred to as the Master switches. Through these parameters we can omit some aspects of the event generation chain. So we turned on only one Pythia Master switch at a time, and the results for the visible cross section are shown in figure 4.7. The results in figure 4.7 for Pythia6 and Pythia 8 are very similar only when initial state radiation (ISR) is turned on. So we can state that the cause of the small variation is somewhere in multiparton interactions (MPI) and final state radiation (FSR). Consequently focusing on the two scenarios separately, 
we simulate events, turning off only one of those master switches. At the end we determined that the culprit is FSR as shown in figure 4.8. For all of our analysis, we use Pythia8 since it gives a better description of the underlying events and it is up to date.

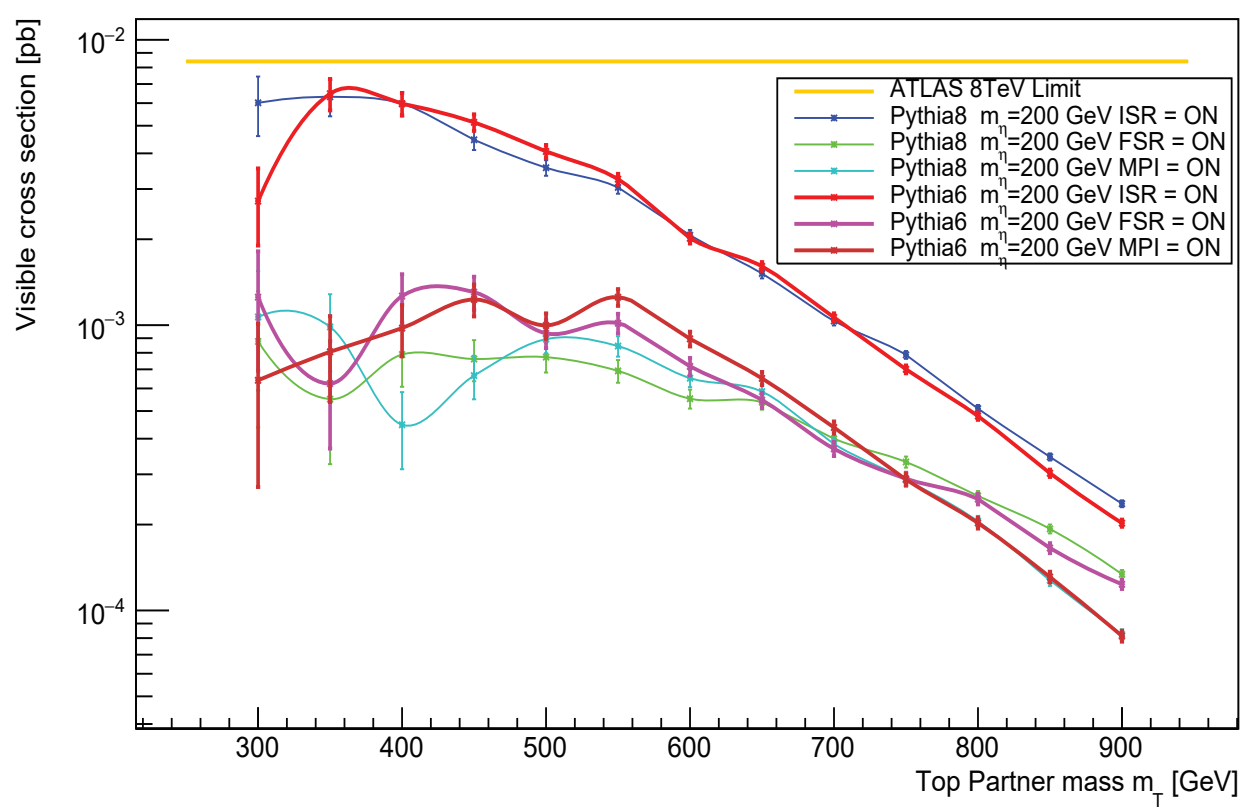

Figure 4.7: The plot shows the cross section for an ATLAS search for Pythia6 vs Pythia8 while turning on only one Pythia Master switch at a time. 


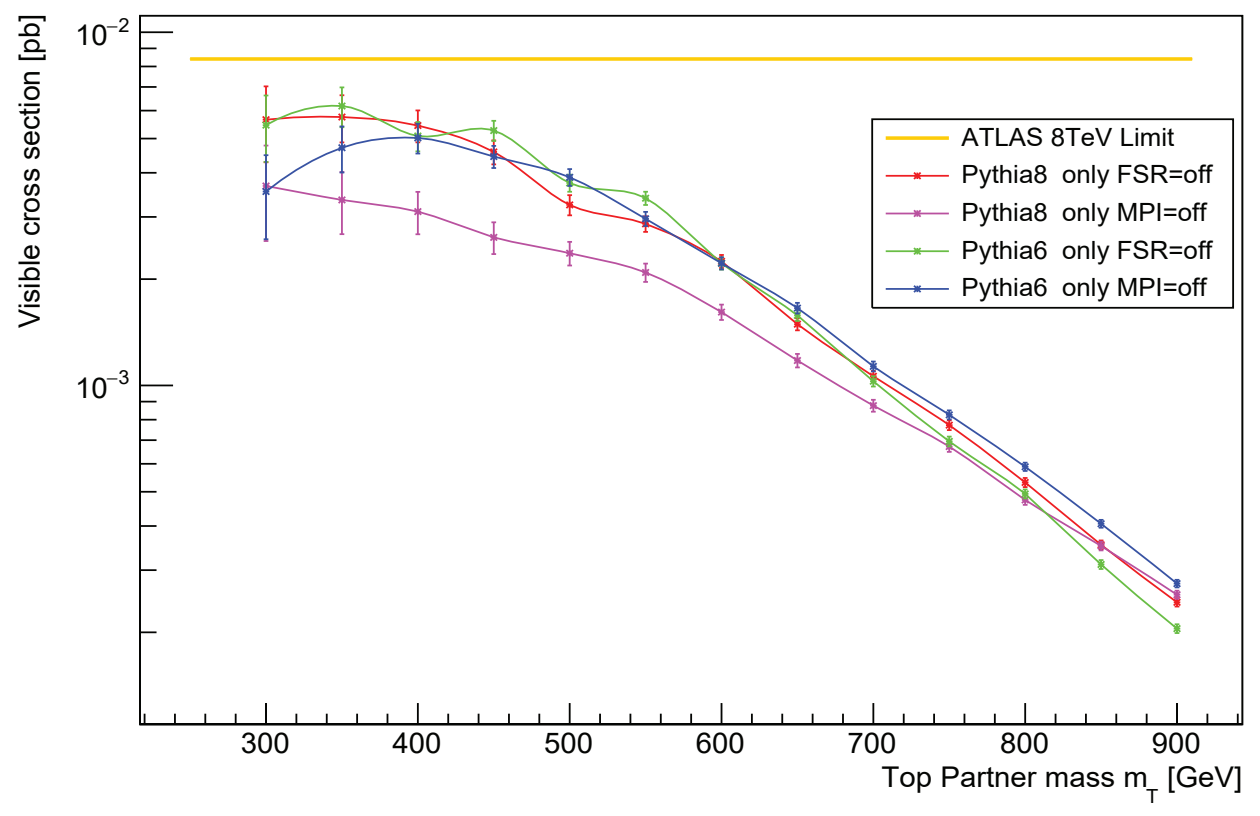

Figure 4.8: The plot shows the cross section for Pythia8 and Pythia6 for an ATLAS analysis with $m_{\eta}=200 \mathrm{GeV}$ while turning off only one Pythia Master switch at a time.

The difference in the two programs is due to the Pythia8 interleaved parton shower with multiparton interaction [23]. The idea behind the interleaved shower is that processes with high scale occur before in comparison to processes with lower scale. 


\section{Chapter 5}

\section{Results and Discussion}

With all the necessary information and tools in hand, we can finally present some results for our model. We will start by discussing the LHC searches that were used over the course of the study. Moreover, we will look at the constraints procured from these experimental searches.

\subsection{Case I: Light Flavour Results}

As discussed previously, the expected limit from the light flavour jets are weaker due to the presence of the large multi-jet QCD background in the signal region. From all the searches considered only the CMS search provides a limit, while the rest of the searches turn out not to.

\subsubsection{CMS Search}

The CMS collaboration has conducted a three jet resonance search [13] at the LHC and we have recasted this search for our model. The selected events must have high transverse momentum and high jet multiplicity. The search reconstructs three jet 
resonances and uses an R-parity violating supersymmetric model, where pair produced gluino decays to three jets through an off-shell squark, as a benchmark. All the cuts applied to events are shown in table 5.1. The search considers two scenarios, first when the gluino decays into light flavour jets and secondly when it decays to a b-jet and two light flavour jets. The latter case requires the existence of at least one bottom quark jet in the resonance decay products. The main feature of the search is the use of a jet ensemble technique, which takes the six highest $p_{T}$ jets in the event and assembles them into all 20 possible unique triplets. Out of all these 20 triplets, at most two will be from the correct reconstruction of the pair produced gluinos. To achieve sensitivity to the three jet resonance, a requirement on the triplet mass is placed in order to reduce the SM background and the incorrectly combined signal triplets. So the triplet invariant mass must satisfy,

$$
M_{j j j}<\left(\sum_{i=1}^{3} p_{T}^{i}\right)-\Delta
$$

The value of $\Delta$ is determined empirically by studying the peak position of $M_{j j j}$ as a function of $\Delta$. From the study of the peak position, the CMS collaboration has determined that $\Delta=110 \mathrm{GeV}$ to be the optimal choice.

To further increase the signal sensitivity, the signal event shape information is exploited. Typically in the high mass region, the signal events have a more spherical shape than the background (which generally contain back to back jets thus more linear shape) [13]. Thus the sphericity variable is defined as,

$$
S=\frac{3}{2}\left(\lambda_{2}+\lambda_{3}\right), \quad \lambda_{1} \geq \lambda_{2} \geq \lambda_{3}
$$


Where $\lambda$ 's are the eigenvalues of the the sphericity tensor,

$$
S^{\alpha \beta}=\frac{\sum_{i} p_{i}^{\alpha} p_{i}^{\beta}}{\sum_{i}\left|p_{i}\right|^{2}}, \quad \alpha, \beta=\mathrm{x}, \mathrm{y}, \mathrm{z}
$$

\begin{tabular}{|c|c|c|c|}
\hline \multirow{2}{*}{$\begin{array}{l}\text { Selection } \\
\text { criteria }\end{array}$} & \multirow{2}{*}{$\begin{array}{c}\text { Inclusive } \\
\text { Search }\end{array}$} & \multicolumn{2}{|c|}{ Heavy Flavour } \\
\hline & & Low mass & High mass \\
\hline Mass range & $400-1500 \mathrm{GeV}$ & $200-600 \mathrm{GeV}$ & $600-1500 \mathrm{GeV}$ \\
\hline$n_{\text {jet }} \geq$ & 6 & 6 & 6 \\
\hline$\Delta=$ & $110 \mathrm{GeV}$ & $110 \mathrm{GeV}$ & $110 \mathrm{GeV}$ \\
\hline$p_{T}^{4 t h-j e t} \geq$ & $110 \mathrm{GeV}$ & $80 \mathrm{GeV}$ & $110 \mathrm{GeV}$ \\
\hline$p_{T}^{6 t h-j e t} \geq$ & $110 \mathrm{GeV}$ & $60 \mathrm{GeV}$ & $110 \mathrm{GeV}$ \\
\hline$S \geq$ & 0.4 & - & 0.4 \\
\hline$n_{b-j e t s} \geq$ & - & 1 & 1 \\
\hline$\left|\eta_{j e t}\right|<$ & \multicolumn{3}{|c|}{2.5} \\
\hline$R=$ & \multicolumn{3}{|c|}{0.5} \\
\hline
\end{tabular}

Table 5.1: The table shows the CMS multi-jet search cuts for various signal regions.

The search has been recasted by first coding all the cuts applied to the events(summarized in table 5.1) in $\mathrm{C}++$ for this and all the other searches. Next we have simulated RPV topology by pair producing gluinos and decaying them via an off-shell squarks(superpartners of quarks) for the light and heavy flavour separately as discussed in [13] and compared the results to [13] to make sure the analysis was successfully replicated. Then we used the analysis on our model and obtained the efficiencies for each point in the $m_{T} m_{\eta}$-plane. The efficiencies obtained for our 
$\operatorname{model}\left(\epsilon_{\text {our }}\right)$ were then used to rescale the production cross section as follows:

$$
\sigma_{\text {rescaled }}=\sigma_{\text {prod }} \times \frac{\epsilon_{\text {our }}}{\epsilon_{\text {obs }}}
$$

Where $\sigma_{\text {prod }}$ is the production cross section for the top $\operatorname{partner}(p p \longrightarrow \bar{T} T)$ at a given center of mass energy and $\epsilon_{o b s}$ is the efficiency given for the benchmark model studied in [13] ${ }^{1}$. So we exclude any point that has $\sigma_{\text {rescaled }}$ greater than the $95 \%$ confidence upper limit given in the CMS paper as a function of the gluino mass.

Now lets look at the $\sqrt{s}=8 \mathrm{TeV}$ CMS search [13] for any possible constraints on our model. In figure 5.1 we plotted the $95 \%$ confidence upper limit on the cross section as a function of the gluino mass from the CMS search (orange curve) and the rescaled cross section for our model as function of the top partner mass for various scalar masses (blue curve for $m_{\eta}=300 \mathrm{GeV}$, cyan curve for $m_{\eta}=200 \mathrm{GeV}$ and red curve for $m_{\eta}=100 \mathrm{GeV}$ ). There appear to be a limit around $m_{T} \approx 350 \mathrm{GeV}$ and also for $m_{T} \approx 500 \mathrm{GeV}$ for some $m_{\eta}$. Consequently our light flavour model is constraint by the CMS analysis.

Performing a parameter scan, we obtained the results shown in figure 5.2. The plot shows the excluded region of the parameter space in $m_{T} m_{\eta}$-plane. The shaded region of the parameter space is excluded by the CMS search. For example, the CMS search has excluded the top partner $m_{T}=350 \mathrm{GeV}$ independent of $m_{\eta}$ and $m_{T}=500$ only for $250 \mathrm{GeV} \leq m_{\eta} \leq 380 \mathrm{GeV}$. As shown in figure 5.2, we have scanned the region $m_{\eta} \geq 100 \mathrm{GeV}$ but it is possible for the scalar to be lighter. During the parameter scan, we have not included the region below $m_{T}=350 \mathrm{GeV}$ for the CMS search because the search considers only the region $m_{\tilde{g}} \geq 350 \mathrm{GeV}$. They don't consider the

\footnotetext{
${ }^{1}$ For the heavy flavour scenario the efficiency,$\epsilon_{o b s}$, was obtained by simulating pair produced gluinos which subsequently decays to a b-quark and two light quarks with all the squarks decoupled.
} 


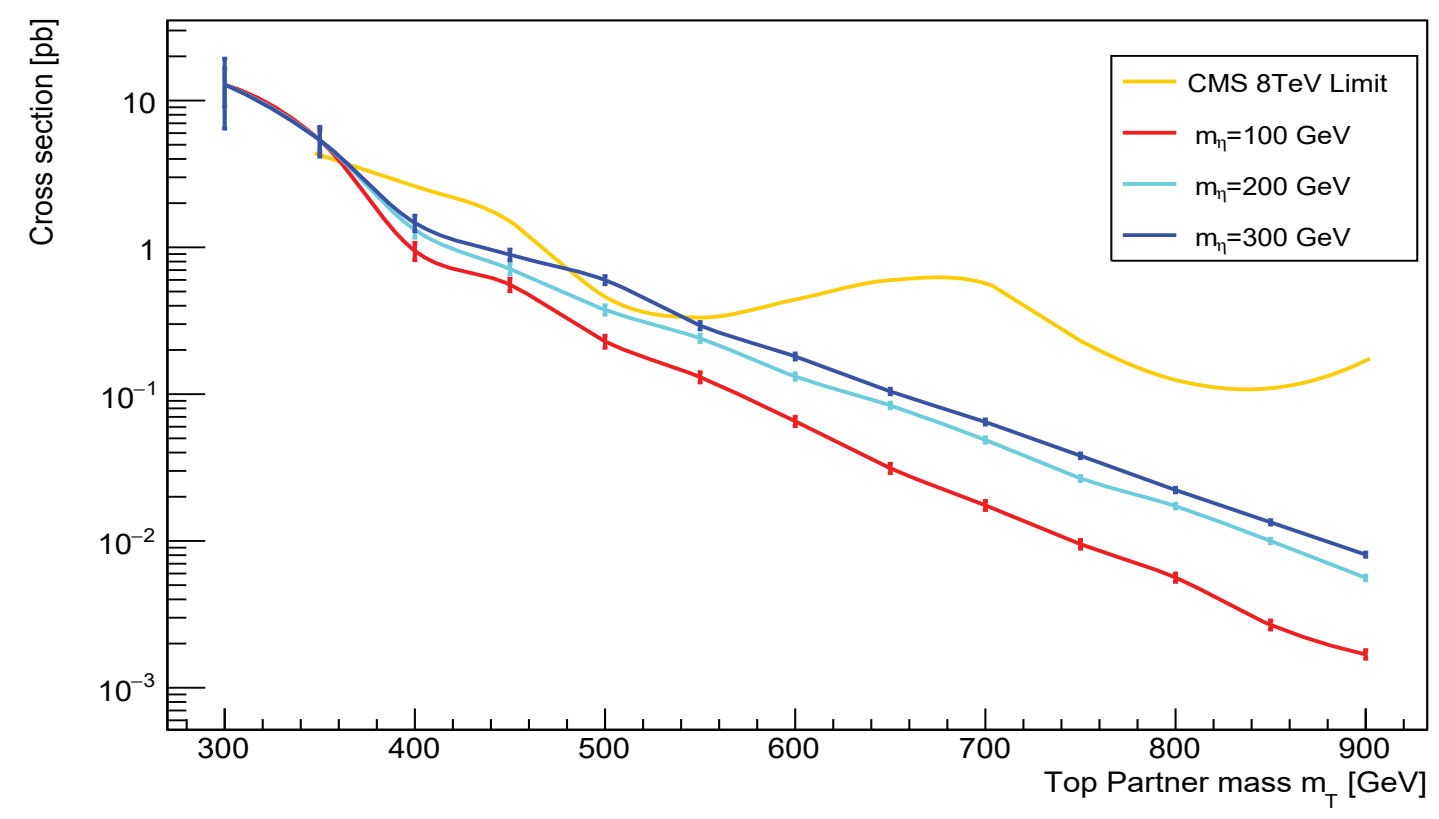

Figure 5.1: The plot shows the rescaled cross section at $\sqrt{s}=8 \mathrm{TeV}$ as a function of the top partner mass for various scalar masses.

region below $350 \mathrm{GeV}$ since their previous search for the same topology has excluded the gluino mass up to $460 \mathrm{GeV}$ [24]. So it is logical for them to examine only the region that has not been excluded yet. However, it would have been very helpful if they had extended their $8 \mathrm{TeV}$ search below $m_{\tilde{g}}=350 \mathrm{GeV}$. Hence we needed to recast the $7 \mathrm{TeV}$ analysis [24] to include the low mass region for the analysis and the result is displayed in figure 5.3. As we can see from the plot, the lower mass region has not been excluded by the $7 \mathrm{TeV}$ CMS search either. 


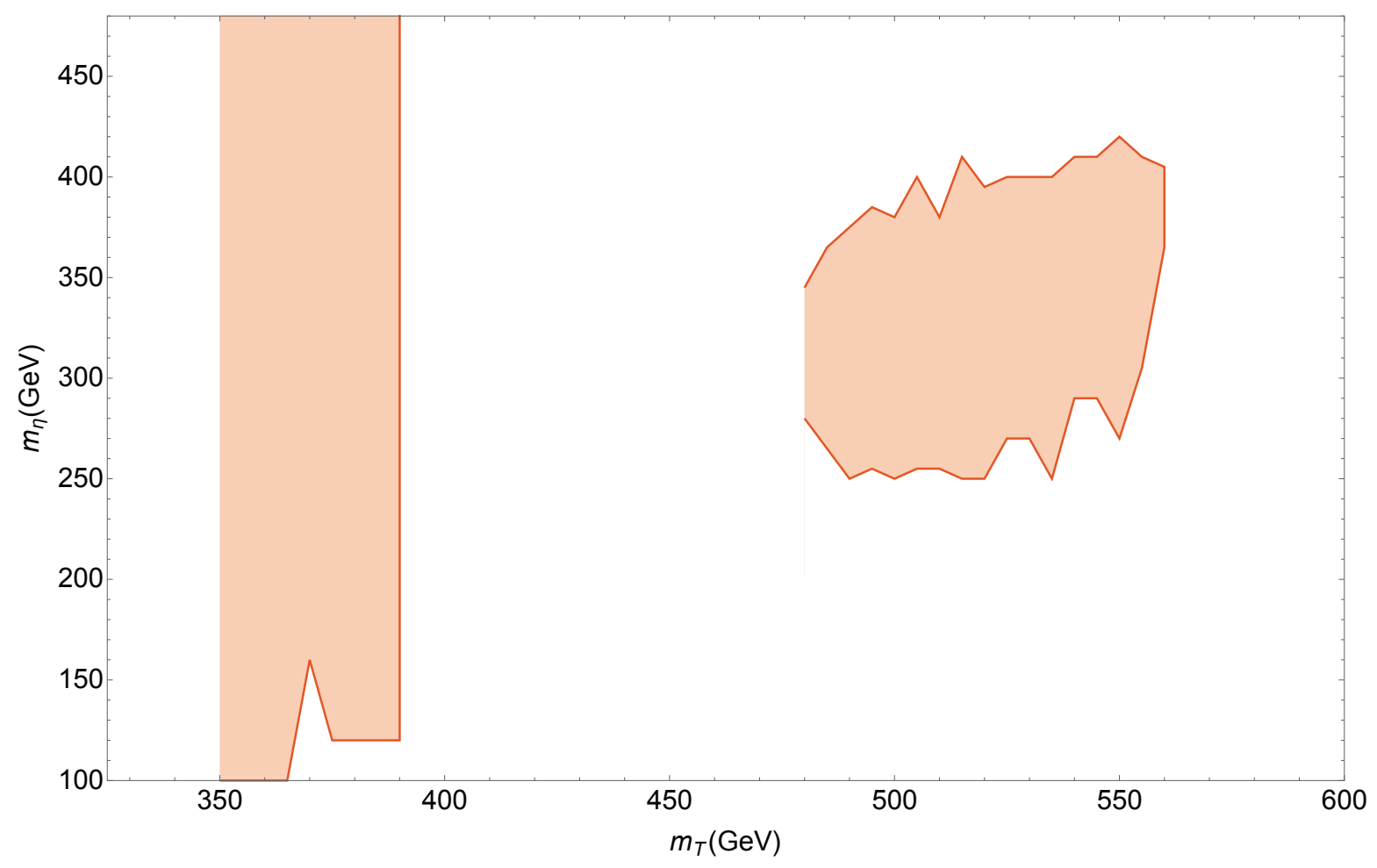

Figure 5.2: Exclusion curve for the top partner decaying into light jets. All the excluded points in the parameter space are represented by the shaded regions.

\subsubsection{ATLAS Search}

Topologies similar to our signal have been explored at the LHC. For example, the ATLAS collaboration has conducted a search for a multi-jet topology at $\sqrt{s}=8 \mathrm{TeV}$ [14] which we have recasted for our model. The search was designed to look for a massive supersymmetric particle decaying into fully hadronic states. Although the search is designed for R-parity violating supersymmetric gluino pair production, the selection criteria applied are model independent and it can be used to constrain similar signal signatures. The analysis is performed by requiring at least 7 jets reconstructed using the anti $-k_{t}$ algorithm with radius parameter of $R=0.4$. All the jets are required to have a transverse momentum of at least $120 \mathrm{GeV}$. The summary of all 


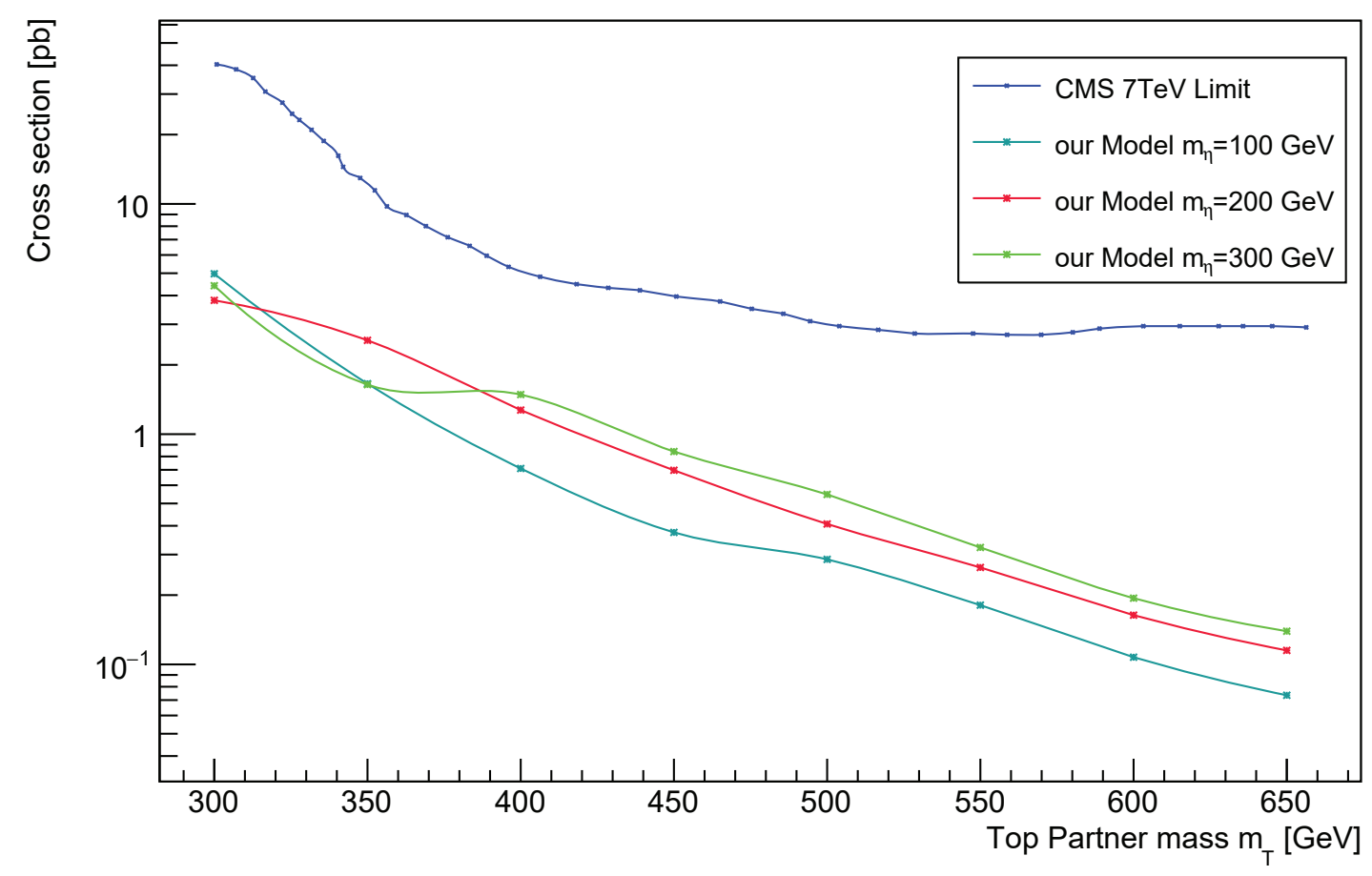

Figure 5.3: The figure displays the rescaled cross section for our model using the 7 $\mathrm{TeV}$ CMS search and the $95 \%$ confidence upper limit on the cross section.

the cuts are presented in table 5.2 .

Jets can be accurately reconstructed if they are well-separated from each other and the beamline, then the jet algorithm can be used to cluster most of the hadrons present in the detector. But in the actual experiment the detector records much more information than just the final state from a hard scattering event and jets are usually not well-separated from each other or the beamline. The incoming partons typically radiates before scattering, leading to copious initial state radiation(ISR). Also the partons may further interact beside the hard scattering event from the colliding hadrons which is referred to as multiparton interactions(MPI). So the aim is to select a patch of calorimeter that contains significant amount of final state radiation 
and reduces the contamination from the initial state radiation (ISR), multiparton interaction (MPI) and pileup. So the overall effect is that the partons from MPI and ISR can spatially overlap with the partons from FSR, thus complicating the jet reconstruction. At the end, there is a trade off. On the one side, we would like our jets to be large enough to include all of the hard scattering decay products. On the other hand, we are restricted to how large the jet can be in order to minimize the contamination resulting from partons unassociated with the hard scattering.

In order to reduce the contamination, one approach is to go inside the jet and remove the radiation contamination from each jet. The basic principle of this method stems from the experimental observation that there is typically only one hard scattering per event and the radiation originating from all the other sources are likely to be much softer. As such the ATLAS search employs a trimming algorithm which uses the ratio of the $p_{T}$ of subjets (jets composed of the constituents of the original jet) to the original jet as a cut and it relies on the fact that the underlying events (secondary interactions between the proton beam remnants) and pileup in the reconstructed jets leads to softer contamination than the hard scattering [25]. All the subjets are reconstructed with a radius parameter $R_{\text {subjet }}=0.3$. We have implemented the jet trimming using FastJet [26], which is $\mathrm{C}++$ package that has fast jet finding and analysis tools.

We recast the above ATLAS search by coding all the cuts applied to the events and simulated their corresponding RPV topology with all the squarks off-shell. As a first step, the results for the RPV topology were compared to the results of [14] to make sure our tools can reproduce the results obtained by the experiment. Afterward, we applied the coded analysis to the events simulated using our model for various $m_{T}$ 


\begin{tabular}{|l|c|}
\hline Variables & Cuts \\
\hline$n_{\text {jet }} \geq$ & 7 \\
$\left|\eta_{\text {jet }}\right|<$ & 2.8 \\
$R=$ & 0.4 \\
$R_{\text {subjets }}=$ & 0.3 \\
$p_{T}^{i}$ & 0.05 \\
$p_{T}^{\text {jet }}$ & \\
\hline \hline Signal Region & Selection Criteria \\
\hline$\left(p_{T}^{\text {jet }}, n_{\text {-tags }}\right)=$ & $(120 \mathrm{GeV}, 0)$ \\
$\left(p_{T}^{\text {jet }}, n_{\text {-tags }}\right)=$ & $(180 \mathrm{GeV}, 0)$ \\
$\left(p_{T}^{\text {jet }}, n_{\text {-tags }}\right)=$ & $(120 \mathrm{GeV}, 1)$ \\
$\left(p_{T}^{\text {jet }}, n_{\text {-tags }}\right)=$ & $(180 \mathrm{GeV}, 1)$ \\
$\left(p_{T}^{\text {jet }}, n_{\text {-tags }}\right)=$ & $(80 \mathrm{GeV}, 2)$ \\
$\left(p_{T}^{\text {jet }}, n_{\text {b-tags }}\right)=$ & $(120 \mathrm{GeV}, 2)$ \\
\hline
\end{tabular}

Table 5.2: The table shows all the cuts applied to the ATLAS multi-jet search performed at $\sqrt{s}=8 \mathrm{TeV}$.

and $m_{\eta}$. The analysis produces the efficiency for a given mass point in the $m_{T} m_{\eta^{-}}$ plane which can be used to calculate the visible $\operatorname{cross} \operatorname{section}\left(\sigma_{\text {vis-our }}\right)$ that is defined as:

$$
\sigma_{\text {vis }} \equiv \frac{N_{\text {non-SM }}}{\mathcal{L}}=\sigma_{\text {prod }} \times \epsilon
$$

Where $N_{n o n-S M}$ is the number of non-SM events and $\epsilon$ is the efficiency of the cuts. Moreover, $\sigma_{\text {prod }}$ is the production cross section and $\mathcal{L}$ is the integrated luminosity $\left(\mathcal{L}=\int L \mathrm{~d} t\right)$. Then using the given observed $95 \%$ confidence upper limits on the visible cross $\operatorname{section}\left(\sigma_{\text {vis-obs }}\right)$ in the ATLAS papers [14,27], we exclude a point if $\sigma_{\text {vis-our }}$ is greater than $\sigma_{\text {vis-obs }}$. 


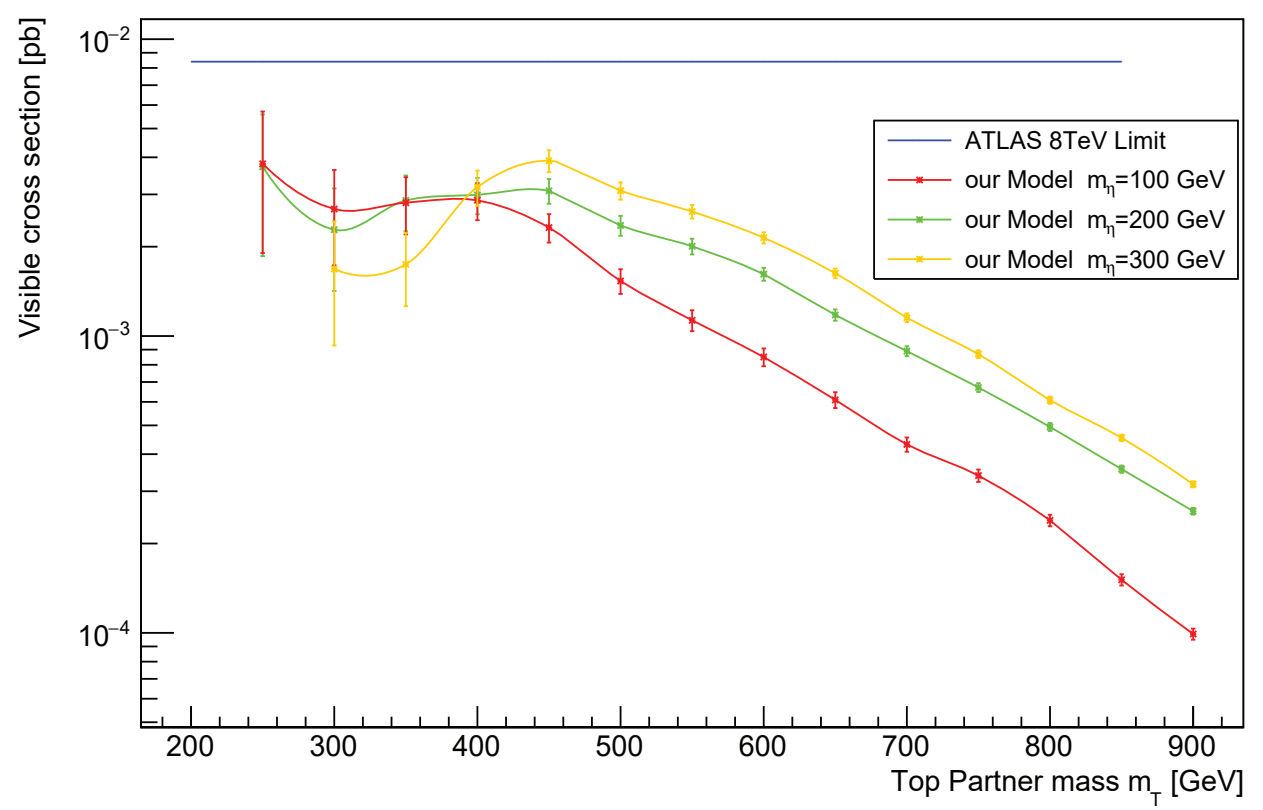

Figure 5.4: The plot displays the visible cross section obtained for the ATLAS search where the top partner decays to light flavour SM quarks. The blue curve represent the $95 \%$ confidence upper limit on the visible cross section from the ATLAS paper.

Applying the ATLAS search to our model, we have obtained the results shown in figure 5.4 where we have plotted the visible cross section as a function of the top partner mass for different $m_{\eta}$ with the $95 \%$ confidence upper limits on the visible cross section from $\sqrt{s}=8 \mathrm{TeV}$ ATLAS search [14]. As shown in figure 5.4, $\sigma_{\text {vis }}$ is quite insensitive to the various $m_{\eta}$ and there seems to be no limit coming from the ATLAS search. In order to fully exploit the features of the model we need to perform a parameter scan in the $m_{\eta}$ and $m_{T}$ plane to get the exclusion curve for the light flavour scenario. We have completed such a scan for the ATLAS analysis but the search has produced no exclusion limit on our desired model. Thus the ATLAS search imposed no constraints on the mass of the top partner. 
Furthermore, there is another ATLAS search at $\sqrt{s}=13 \mathrm{TeV}$ [27] that is built upon the $8 \mathrm{TeV}$ search. The search looks for pair produced gluinos with subsequent R-parity violating decay to three quarks and it has excluded gluinos masses less than $1080 \mathrm{GeV}$. The search uses $14.8 \mathrm{fb}^{-1}$ of data collected by the ATLAS detector. The decay topology is shown in figure 4.1. The search requires events with at least four large radius jets $(R=1.0)$ with a transverse momentum of at least $200 \mathrm{GeV}$. The analysis requires that the leading jet carries a transverse momentum of at least 440 GeV. Also, the same trimming procedure as discussed in the $8 \mathrm{TeV}$ ATLAS search is applied here but with the sub-jet radius parameter of $R_{\text {sub-jet }}=0.2$. There is an additional selection requirement on the scalar sum of the large radius jet $(R=1.0)$ masses of the event, which is given as,

$$
M_{J}^{\Sigma}=\sum_{\substack{p_{T}>200 \mathrm{GeV} \\|\eta| \leq 2.0}}^{4} m^{j e t}
$$

The event selection cuts for the $13 \mathrm{TeV}$ search are summarized in table 5.3.

We have recasted this $13 \mathrm{TeV}$ ATLAS search [27] but the constraints are extremely weak (shown in figure 5.5 and 5.6) compared to the $8 \mathrm{TeV}$ search. 


\begin{tabular}{|l|c|}
\hline Variables & Selection Requirement \\
\hline$p_{T}^{\text {lead jet }}=$ & $440 \mathrm{GeV}$ \\
$p_{T}^{\text {jets }}>$ & $200 \mathrm{GeV}$ \\
$\left|\eta_{\text {jet }}\right|<$ & 2.0 \\
$\left|\Delta \eta_{12}\right|<$ & 1.4 \\
$R=$ & 1.0 \\
$\Delta R_{\text {subjets }}=$ & 0.2 \\
$p_{T}^{i}>$ & 0.05 \\
\hline \hline$p_{T}^{\text {jet }}>$ & Selection Criteria \\
\hline$\left(n_{\text {jet }}, n_{b-\text { tags }}, M_{J}^{\Sigma}\right)=$ & $(4,0,800 \mathrm{GeV})$ \\
$\left(n_{\text {jet }}, n_{b-\text { tags }}, M_{J}^{\Sigma}\right)=$ & $(4,1,800 \mathrm{GeV})$ \\
$\left(n_{\text {jet }}, n_{b-\text { tags }}, M_{J}^{\Sigma}\right)=$ & $(5,0,600 \mathrm{GeV})$ \\
$\left(n_{\text {jet }}, n_{b-\text { tags }}, M_{J}^{\Sigma}\right)=$ & $(5,1,600 \mathrm{GeV})$ \\
\hline
\end{tabular}

Table 5.3: The table displays the ATLAS 13 TeV multi-jet search selection requirement for the events.

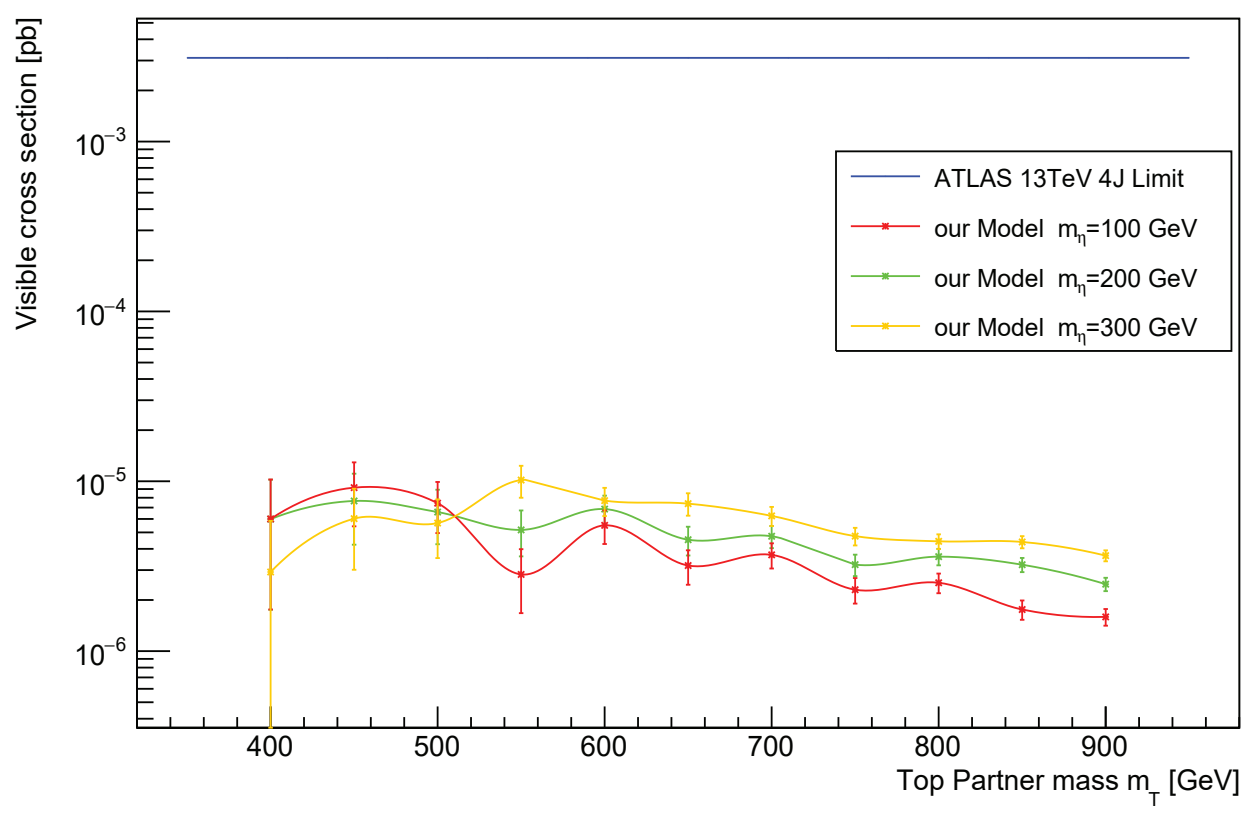

Figure 5.5: The figure shows the visible cross section as a function of the top partner mass for the $\sqrt{s}=13 \mathrm{TeV}$ ATLAS search with at least four jets $\left(N_{\text {jet }} \geq 4\right)$ in the final state. 


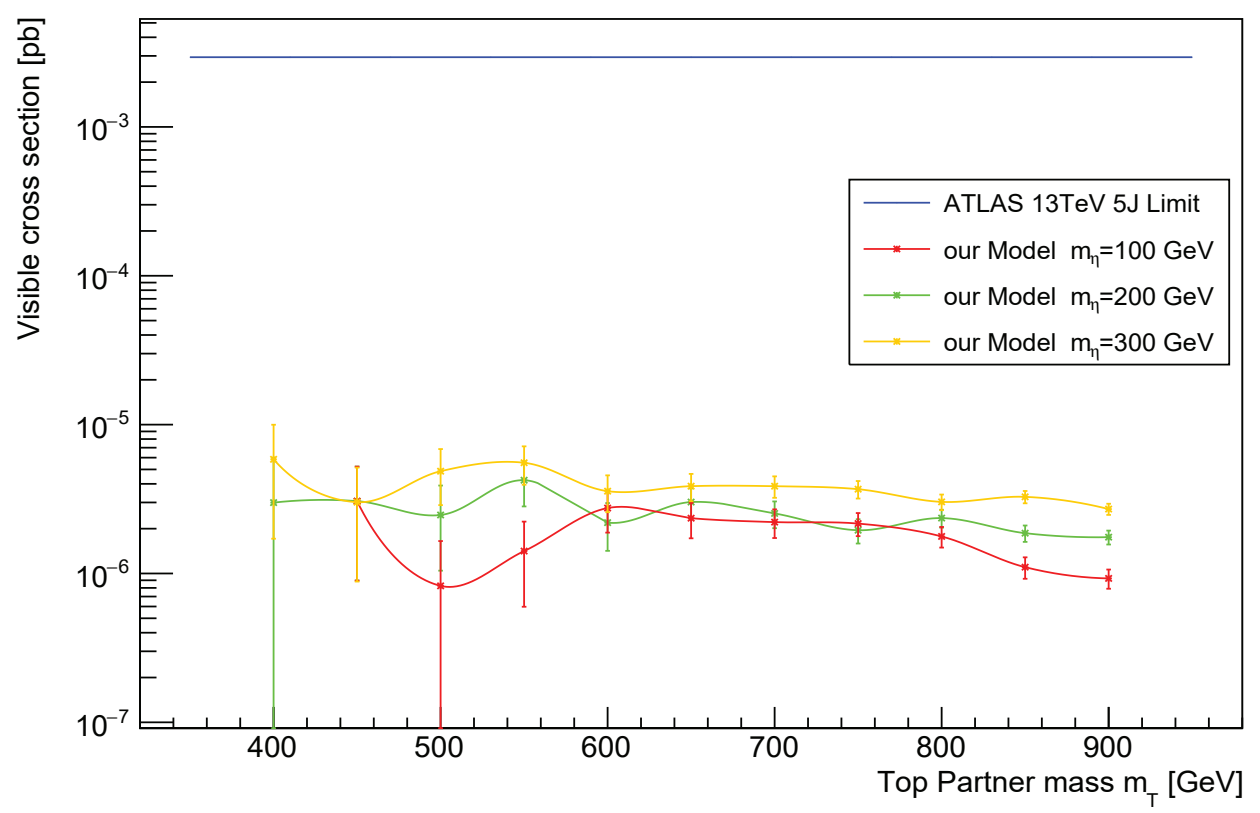

Figure 5.6: The figure shows the visible cross section as a function of the top partner mass for the $\sqrt{s}=13 \mathrm{TeV}$ ATLAS search with at least five jets $\left(N_{\text {jet }} \geq 5\right)$ in the final state.

Now one might wonder why is the signal more sensitive to the CMS analysis compared to the ALTAS? Well the ATLAS search relies mainly on the transverse momentum of the jets to discriminate between the signal and background. On the other hand, the CMS search actually reconstructs the correct three jet resonances for the signal region and combines that with the selection on the jets transverse momenta which is in fact a more robust method for our signal topology.

\subsection{Case II: Heavy Flavour Results}

Here the Top partner is coupled to the SM top quark and a new scalar particle that decays to the SM up quarks. The signal topology is shown in figure 3.3 which is 
similar to the CMS search described in the next section 5.2.1.

\subsubsection{CMS Leptonic Search}

A RPV supersymmetric search [12] has been recently conducted by the CMS collaboration that explores final states with leptons + jets. The search considered looks for pair produced gluinos at $\sqrt{s}=8 \mathrm{TeV}$ at the LHC where each gluino then decays directly to one top and two light quarks as shown in figure 5.7. The search explores the possibility for $\tilde{g} \longrightarrow t b s$ events in which the top quark undergoes a semi-leptonic decay. The analysis performed uses the data collected by the CMS collaboration corresponding to an integrated luminosity of $19.5 \mathrm{fb}^{-1}$. No excess of events above the Standard Model prediction has been observed so a 95\% confidence level limit on the production cross section of the supersymmetric particle has been established. The leptonic analysis requires at least one lepton (electron or muon) with transverse momentum of at least $35 \mathrm{GeV}$ and at least 6 jets with a transverse momentum of at least $30 \mathrm{GeV}$. Furthermore, there are also b-tagging requirements on the final state events ranging from 1 to 5 . The summary of all the selection requirements on the events is shown in table 5.4.

This search was applied to our model for various scalar masses $\left(m_{\eta}\right)$ and top partner $\operatorname{masses}\left(m_{T}\right)$ in the range $[100,1000] \mathrm{GeV}$ and the number of non-SM events were calculated as follows:

$$
N_{\text {Non-SM }}^{\text {our }}=\sigma_{\text {prod }} \times \mathcal{L} \times \epsilon
$$

Where $\sigma_{\text {prod }}$ is the production cross section of the top $\operatorname{partner}(\sigma(p p \longrightarrow \bar{T} T)), \mathcal{L}$ is the integrated luminosity and $\epsilon$ is the efficiency of the applied cuts. Furthermore, 
given the number of observed events and their uncertainty given in the CMS paper [12] for each of the signal regions (the signal region is binned based on the number of jets 6-8, the number of b-tags 1-5 and lepton flavour), we can calculate the number of non-SM events in each bin using the $C L_{s}[28]$ method that we have implemented in MATHEMATICA:

$$
C L_{s}=\frac{C L_{s+b}}{C L_{b}}
$$

Where $C L_{s+b}$ is the confidence level for excluding the simultaneous presence of signal and background events and $C L_{b}$ is the possibility of observing events less than or equal to the number of events assuming only the background hypothesis (for more detail refer to appendix A.5). Then to quote a $95 \%$ confidence upper limit on the number of events, we vary the number of signal events, $s$, until $C L_{s}=0.05$ and this is repeated for all the bins in the leptonic search. Finally, if $N_{\text {Non-SM }}^{\text {our }}$ is greater than the $95 \%$ upper limit on the number events $\left(N_{\mathrm{Non}-\mathrm{SM}}^{\text {obs }}\right)$ given in [12] that point in our parameter space is excluded by the CMS search.

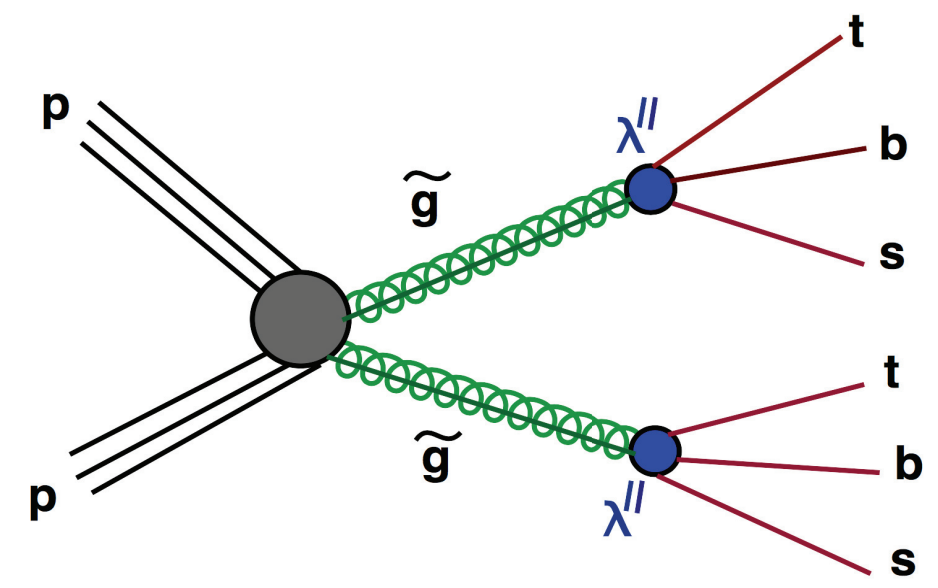

Figure 5.7: Diagram for pair produced gluinos which subsequently decays to tbs. 


\begin{tabular}{|c|c|}
\hline Selection criteria & Selection requirements \\
\hline$p_{T, \text { Jet }}=$ & $30 \mathrm{GeV}$ \\
$p_{T, \text { Lepton }}=$ & $35 \mathrm{GeV}$ \\
$n_{\text {Lepton }}=$ & 1 \\
$\left|\eta_{\text {Lepton }}\right|<$ & 2.5 \\
$\left|\eta_{\text {Jet }}\right|<$ & 2.5 \\
$n_{\text {jet }} \geq$ & $6,7,8$ \\
$n_{\text {bjet }} \geq$ & $1,2,3,4,5$ \\
\hline $\mathrm{R}=$ & 0.5 \\
\hline
\end{tabular}

Table 5.4: The table shows the various selection criteria for the leptonic CMS analysis.
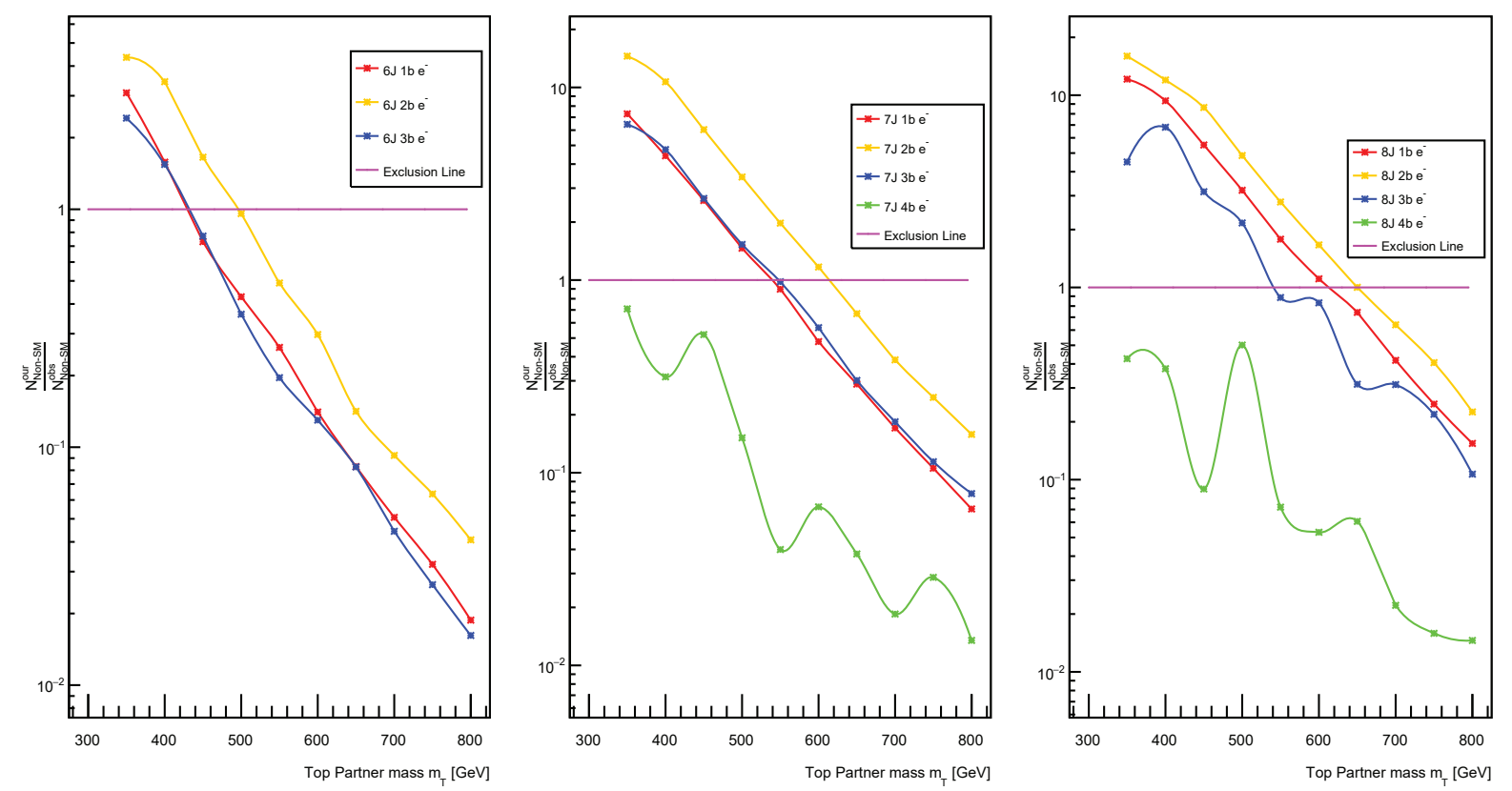

Figure 5.8: The ratio of the number events as a function of the top partner mass for the leptonic CMS search performed at $\sqrt{s}=8 \mathrm{TeV}$. The search require an electron using the scalar $m_{\eta}=200 \mathrm{GeV}$. 
Applying the semi-leptonic search to our model, we obtain a sample $\left(m_{\eta}=200\right.$ $\mathrm{GeV}$ and $\left.m_{T} \in[350,800] \mathrm{GeV}\right)$ of the results as shown in figure 5.8 and 5.9. The figures show the ratio $\left(\frac{N_{\mathrm{N} \text { on }}^{\text {oum }} \text { No }}{N_{\mathrm{Non}-\mathrm{SM}}}\right)$ of the number of non-SM events given by our model to the number of non-SM events observed calculated using information given in the CMS paper [12] as a function of the top partner mass. As shown in the figures, the strongest limits are obtained requiring at least 8 jets with one lepton and 1 or 2 btagged jets. This is expected since b-tagging, and especially requiring a lepton in the final state significantly reduces the background from the Standard Model processes.
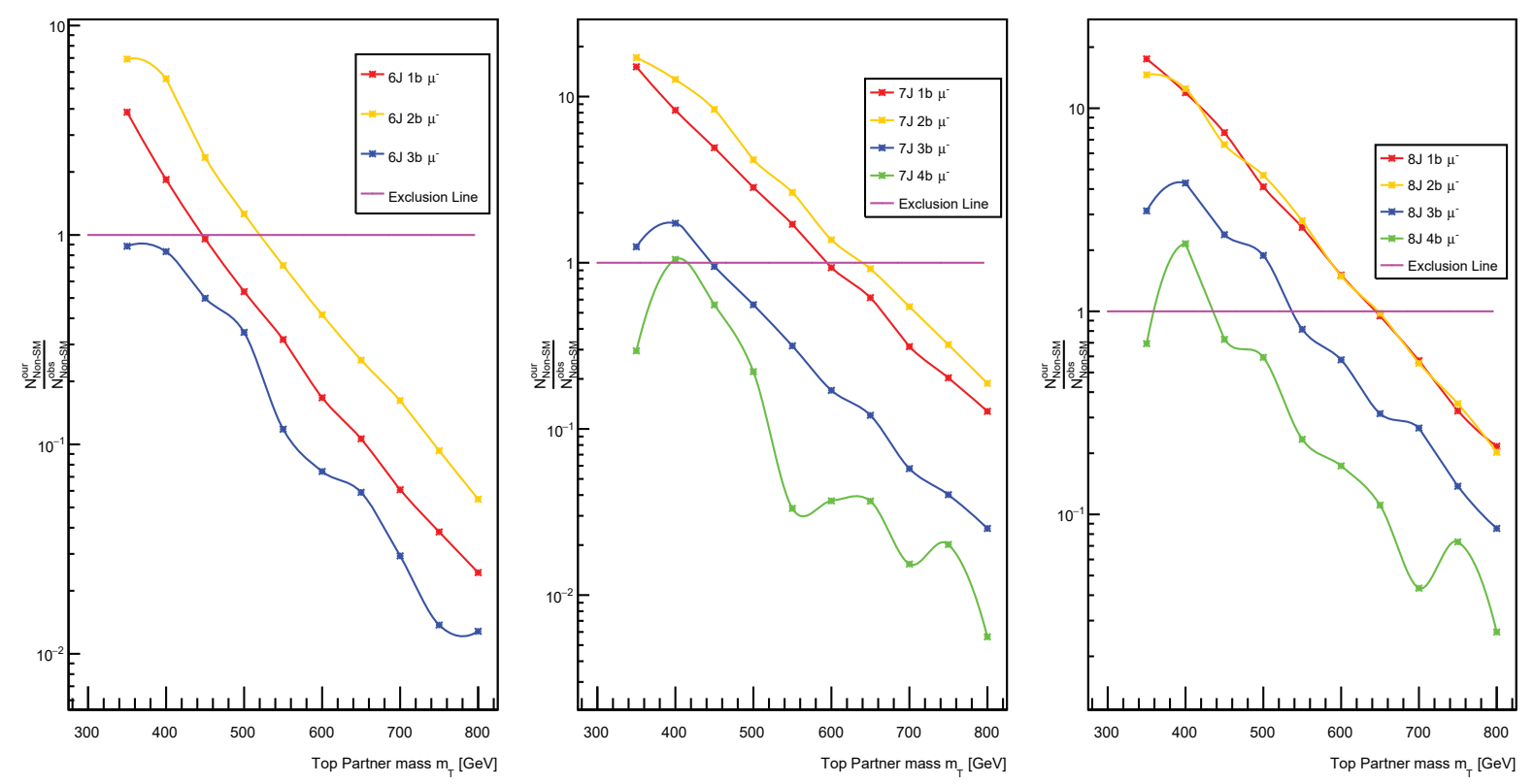

Figure 5.9: The ratio of the number events as a function of the top partner mass for the leptonic CMS search performed at $\sqrt{s}=8 \mathrm{TeV}$. The search require a muon using the scalar $m_{\eta}=200 \mathrm{GeV}$. 
Performing a parameter scan for the leptonic search in the $m_{T} m_{\eta}$-plane, we obtained figure 5.10. The plot shows the allowed(not shaded) and excluded(shaded) region in the $m_{\eta}$ versus $m_{T}$ plane. All the points in the parameter space located in the shaded region are excluded by the leptonic analysis. The exclusion curve was obtained by combining all the signal regions ( signal region requiring jets from 6 to 8, b-tagging from 1 to 5 and different lepton flavour ) to give a stronger limit on the model. As shown in figure 5.10, the lower limit on the top partner mass $m_{T}$ can be as high as $650 \mathrm{GeV}$. This is still less constraining compared to the typical limits obtained for the top partner model exploited at the LHC (as mentioned previously). For the top partner decay to heavy flavour quark, figure 5.10 gives the strongest limits compared to the hadronic analysis (section 5.2.2 and 5.2.3).

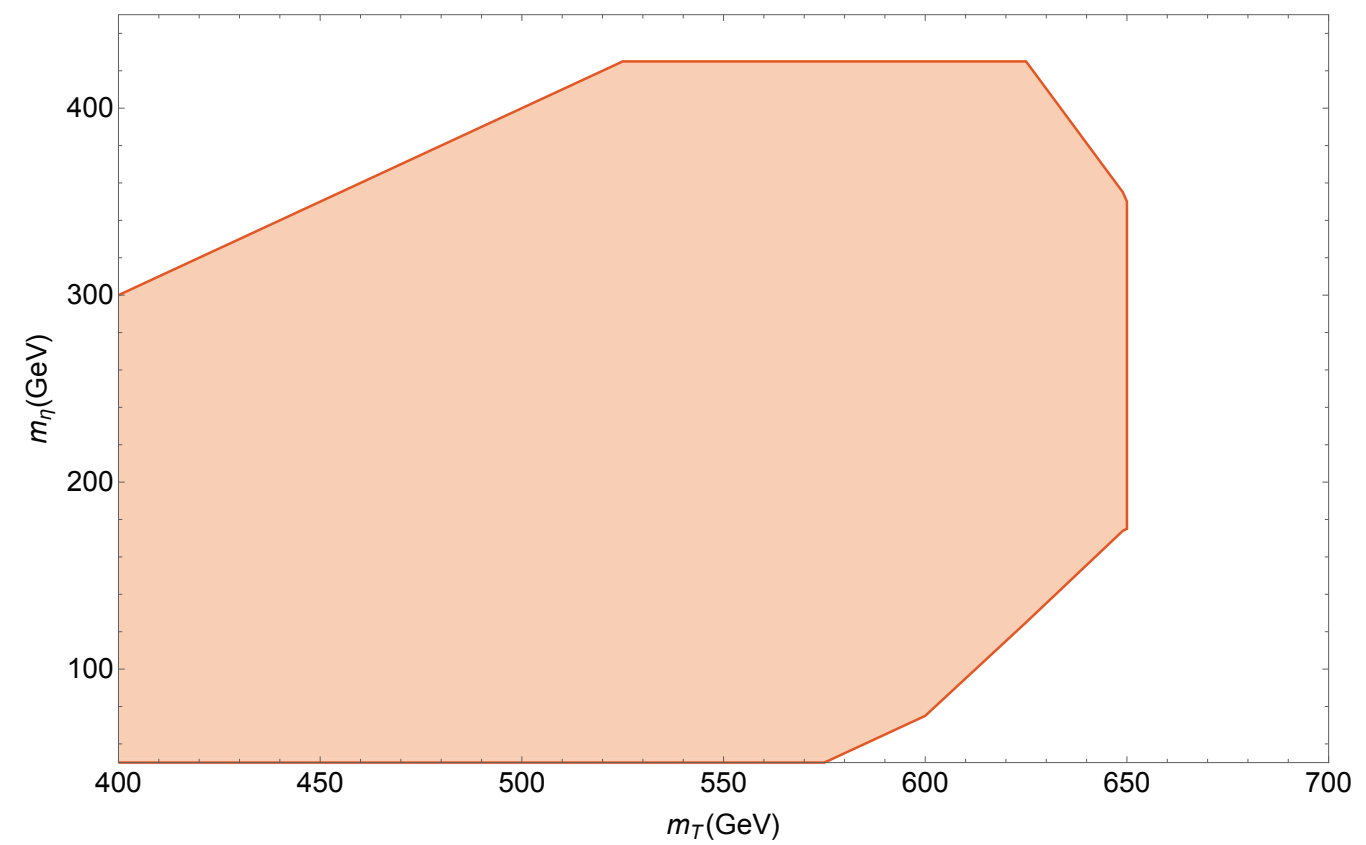

Figure 5.10: The figure shows the exclusion curve using the lepton analysis for the top partner decay to a top quark and two light SM quarks. 


\subsubsection{CMS Hadronic Search}

The CMS collaboration has performed a search at $\sqrt{s}=8 \mathrm{TeV}$ [13] that focuses on a gluino decaying into two light flavour jets and a heavy flavour jet (b-quark). The analysis targets three jet resonances and requires at least one jet from the resonance to be identified as a b-quark jet. The analysis split the gluino mass region into a low $\operatorname{mass} \operatorname{region}\left(m_{\tilde{g}} \in[200,600] \mathrm{GeV}\right)$ and a heavy mass region $\left(m_{\tilde{g}} \in[600,1500] \mathrm{GeV}\right)$. In the low mass region, the fourth and six jet must have $p_{T}$ of at least $80 \mathrm{GeV}$ and $60 \mathrm{GeV}$ respectively. On the hand for the high mass region, the $p_{T}$ of the fourth and sixth jet has to be at least $110 \mathrm{GeV}$. All the cuts are summarized in table 5.1 and all the selection requirements are described in section 5.1.1. The topology is similar to our study and recasting it gives the results shown in figure 5.11.

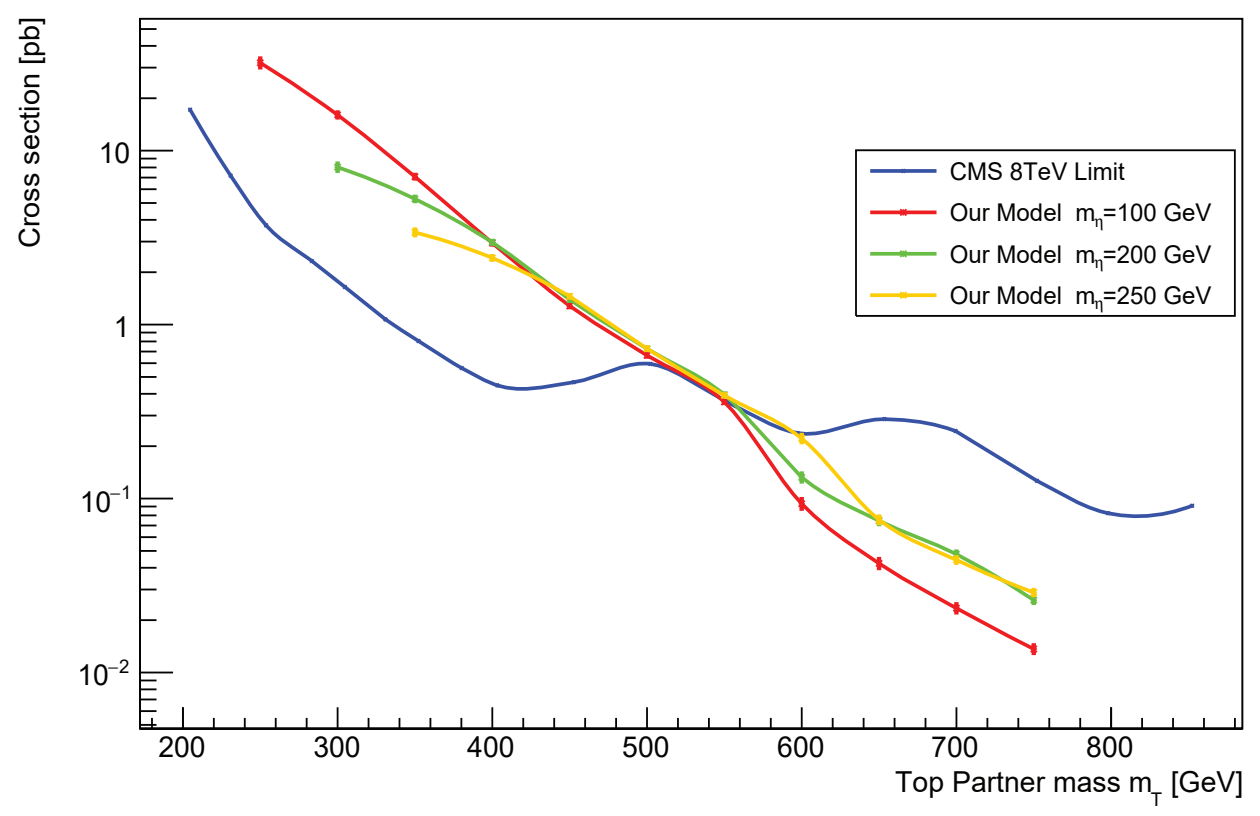

Figure 5.11: The rescaled cross section as a function of the top partner mass for the $\sqrt{s}=8 \mathrm{TeV}$ CMS search with varying $m_{\eta}$. 
The plots shows the 95\% confidence upper limit on the cross section obtained from [13] and the rescaled cross section for our model as a function of the top partner mass for some scalar masses. One thing to notice immediately is that the the rescaled cross section changes very slightly for different scalar masses. This search imposes weak constraints compare to the leptonic CMS search discussed in section 5.2.1. Furthermore, the constraints are stronger in comparison to the light flavour case and the lower limit on the top partner mass is about $m_{T} \approx 550 \mathrm{GeV}$.

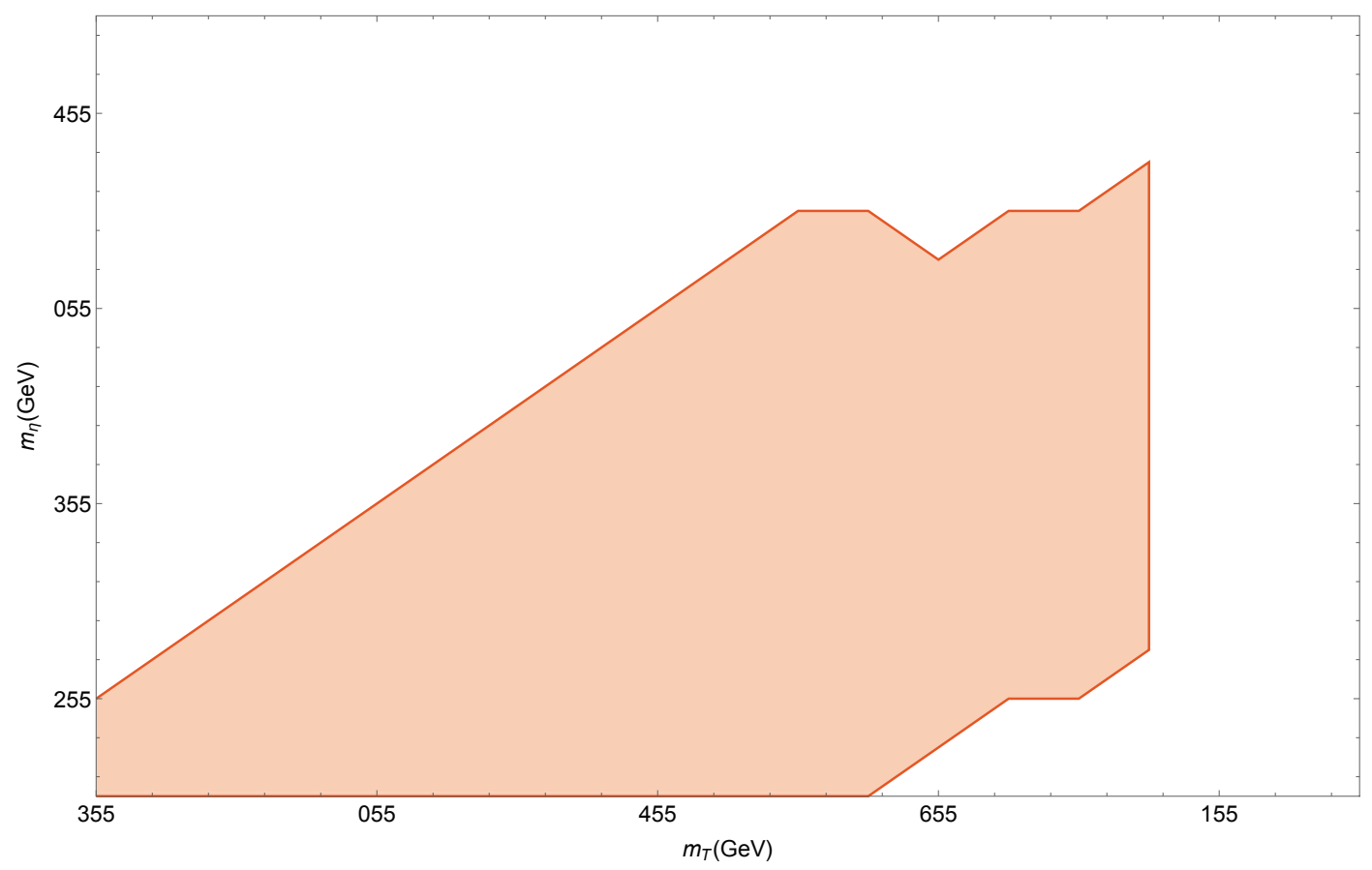

Figure 5.12: The figure shows the exclusion curve from the fully hadronic CMS analysis for the top partner decay to a heavy and two light jets.

To complete the analysis, we again need to perform a parameter scan in the $m_{T} m_{\eta}-$ plane. The exclusion curve for the heavy flavour top partner decay mode is presented in figure 5.12. Comparing figure 5.12 to figure 5.10, we notice that the region of parameter space excluded by the hadronic search is a subset of the region 
excluded by the leptonic analysis. Thus the leptonic search gives stronger limits for our model.

\subsubsection{ATLAS Hadronic Search}

The ATLAS collaboration has conducted a search at $\sqrt{s}=8 \mathrm{TeV}$ [14] that explores the heavy flavour scenario where each of the pair produced gluino decays to a b-quark and two light quarks. The events require at least six jets and the presence of at least one b-jet. All the jets are required to have transverse momentum of at least $80 \mathrm{GeV}$. All the cuts are summarized in table 5.3. Applying this search to our model produces the result shown in figure 5.13.

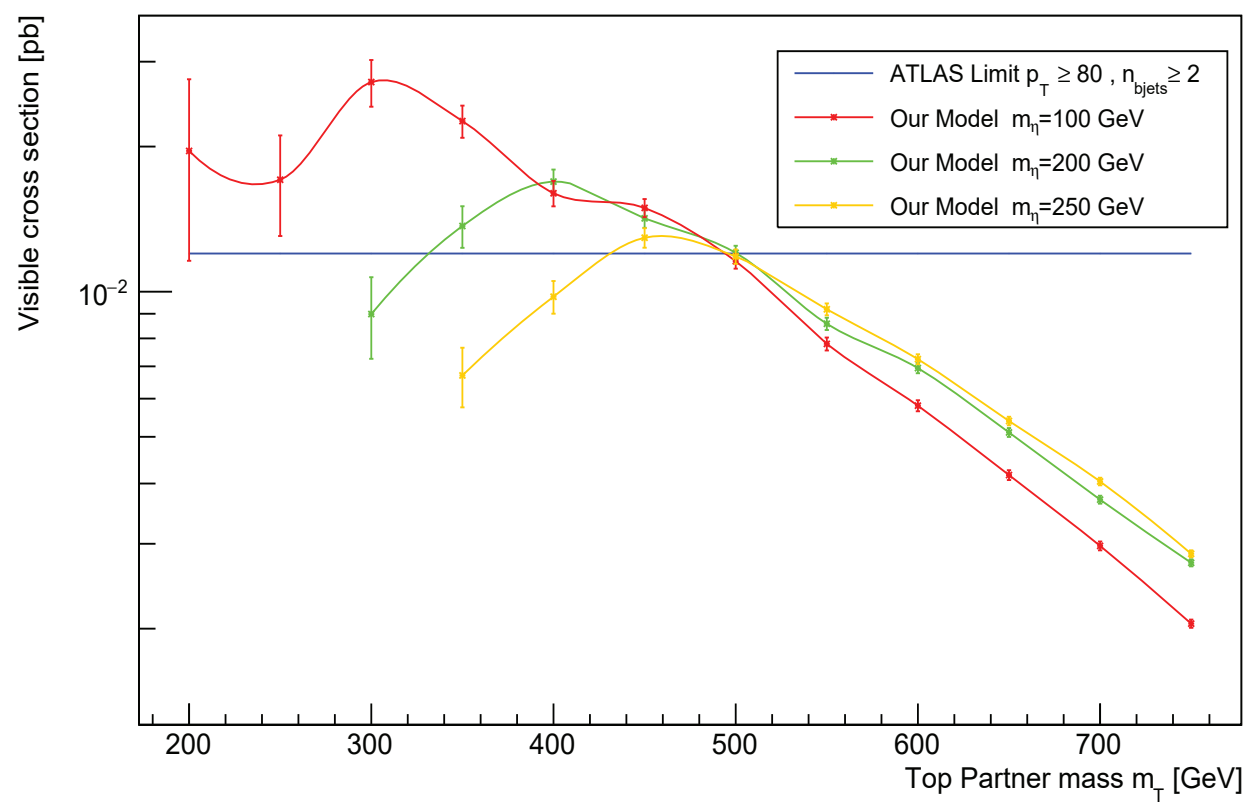

Figure 5.13: The plot shows the visible cross section and the observed $95 \%$ confidence limit on the visible cross section for the heavy flavour top partner with varying $m_{\eta}$.

The figure shows the visible cross section as a function of the top partner mass 
for various $m_{\eta}$. The lower limit from this search on the top partner mass is about $m_{T} \approx 500 \mathrm{GeV}$. One reason for the stronger constraints, compare to the fully hadronic light flavour scenario, is the existence of the b-jets in the events, so we can apply the b-tagging algorithm to identify these jets and count those as signal. Thus b-tagging improves the discrimination between signal and background and it provides us with a better handle on determining signal events.

We have performed a parameter scan for this ATLAS search but the exclusion curve obtained was even weaker than figure 5.12. So the important point is that the CMS leptonic search [12] imposes the strongest constraint on our model as presented in figure 5.10 . 


\section{Chapter 6}

\section{Conclusion}

Fermionic top partners are well motivated theoretically and are expected to be within the reach of the LHC . The aim of this thesis was to examine unexplored decay modes for the top partner. In particular, the two decay topologies studied are: $T \longrightarrow c \eta(\eta \longrightarrow \bar{u} u)$ and $T \longrightarrow t \eta(\eta \longrightarrow \bar{u} u)$. First, we require that the top partner primarily couples to the light up-type quarks and assume that the coupling to all the other SM particles is forbidden or extremely suppressed. The collider signature for this scenario is fully hadronic resulting in multiple jets. Here the available experimental searches have excluded a very small parameter space as shown in figure 5.2. The search has excluded the top partner mass $m_{T}=350 \mathrm{GeV}$ regardless of $m_{\eta}$ in the $m_{T} m_{\eta}$-plane. The top partner masses $m_{T}<350 \mathrm{GeV}$ are not ruled out. However, the Collider Detector at Fermilab (CDF) experimental collaboration has conducted a multi-jet search [29] that puts a lower limit on the the top partner mass $m_{T} \approx 100$ $\mathrm{GeV}$. We are not really certain of this since the limit was obtained from a simple top partner production cross section comparison to the upper limit on the cross section obtained in [29].

Second, we couple the top partner to the SM top quark and a new scalar $(\eta)$ which is only allowed to decay into the SM light quarks. For such a possibility, the collider 
can result in fully hadronic or semi-leptonic topologies. The exclusion plot obtained for this scenario (we refer to it as heavy flavour) is presented in figure 5.10. The strongest exclusion limits come from the semi-leptonic searches and it is expected since the b-tagging and lepton requirement is very important for reducing the multijet background. The lower limit on the top partner mass can be as high as $m_{T} \approx 650$ $\mathrm{GeV}$.

As discussed previously, our model may also provide an alternate solution to the fine-tuning problem in the SM. If the top partner belongs to the light flavour scenario then the fine-tuning required would be very small. However, if the top partner is found in the heavy flavour decay channel then the fine tuning would be somewhat large but still significantly smaller than the suggested SM Planck scale. In both cases under investigation, we found that the current collider lower bounds on the top partner mass are less constraining than the generic decay topologies examined at the LHC $[5,6]$. This is important since less constraining bounds on the top partner mass results in a more natural Higgs mass; consequently, less fine-tuning. In conclusion, hopefully future experiments will focus on the topologies related to our model. 


\section{Appendix A}

\section{Mathematical Tools}

\section{A.1 $\mathbb{Z}_{2}$ Symmetry}

Lets begin with the definition of a group.

Definition 1. Group is a set $G$ together with a binary operation o that satisfies the following axioms,

1. Closure: For $\forall g_{1}, g_{2} \in G$ then $g_{1} \circ g_{2} \in G$

2. Identity: There $\exists$ an element of the group $e \in G$ such that for $\forall g \in G$ then $e \circ g=g \circ e=g$

3. Associativity: For $\forall g_{1}, g_{2}, g_{3} \in G$ then $\left(g_{1} \circ g_{2}\right) \circ g_{3}=g_{1} \circ\left(g_{2} \circ g_{3}\right)$

4. Inverses: For every $g \in G$, there $\exists$ an inverse element of the group $g^{-1} \in G$ such that $g \circ g^{-1}=g^{-1} \circ g=e$

Furthermore by definition, a symmetry is invariance under some defined set of transformations. So $\mathbb{Z}_{2}$ is a cyclic group of order 2 . For $\mathbb{Z}_{n}$, the group elements are easily 
obtained by finding the $n^{\text {th }}$ root of the unity. So

$$
\begin{array}{r}
z^{n}=1, \quad n \in \mathbb{Z}_{+}, \quad \text { So the } n^{t h} \text { root is } z^{\frac{1}{n}}=e^{\frac{i}{n}(\theta+2 \pi m)}, m=0,1,2, \ldots \\
\text { For distinct roots, the value of " } \mathrm{m} \text { " is restricted to } 0 \leq m \leq n-1
\end{array}
$$

Then for $\mathrm{n}=2$, the values of are: $\mathrm{m}=0,1$. Hence the roots are:

$$
\begin{array}{r}
m=0: e^{\frac{i}{2} 2 \pi \cdot 0}=1 \\
m=1: e^{\frac{i}{2} 2 \pi \cdot 1}=-1 \\
\text { Thus, } \mathbb{Z}_{2}=\{-1,+1\}
\end{array}
$$

\section{A.2 Standard Model Groups}

First, the group $\mathrm{U}(1)$ is a unitary group $\left(U^{\dagger} U=U U^{\dagger}=1\right)$ of degree 1 which consists of one-dimensional unitary matrices. Or simply, the group consists of all elements of unit circle in $2 \mathrm{D}$ complex plane.

$$
U(1)=\{z \in \mathbb{C}:\|z\|=1\}, \quad \text { which is satisfied by } z=e^{i \theta}
$$

Secondly, $\mathrm{SU}(2)$ is the special unitary group (matrices of determinant 1) of order 2 , which consists of all unitary matrices with determinant equal to 1 . Now before we write down the most general group structure, it is easier to work with the corresponding Lie algebra of the group. In general, a Lie group is a group that is a smooth manifold (a topological space that locally resembles Euclidean space near each point). Lie group is composed of continuous symmetries. For a Lie group G (order n), the Lie algebra $\mathfrak{g}$ of $\mathrm{G}$ is given by $n \times n$ matrices $\lambda$ such that $e^{\alpha \lambda} \in G$ for $\alpha \in \mathbb{R}$.

Definition 2. Lie algebra is a vector space with a binary operation, Lie Bracket, $([\star, \star]: \mathfrak{g} \times \mathfrak{g} \longrightarrow \mathfrak{g})$ such that it satisfies the following three axioms: 
1. Bilinearity

$$
\begin{aligned}
{[m x+n y, z] } & =m[x, z]+n[y, z] \\
{[x, m y+n z]=m[x, y]+n[x, z] \quad \forall m, n } & \in \mathbb{R}, \forall x, y, z \in \mathfrak{g}
\end{aligned}
$$

2. Anti-commutativity

$$
[x, y]=-[y, x] \quad \forall x, y \in \mathfrak{g}
$$

3. Jacobi Identity

$$
[x,[y, z]]+[y,[z, x]]+[z,[x, y]]=0 \quad \forall x, y, z \in \mathfrak{g}
$$

The Lie bracket for any element of the Lie algebra is defined as:

$$
[x, y] \equiv x y-y x
$$

So all the information of the Lie algebra is encoded in its Lie bracket. Thus returning to $\mathrm{SU}(2)$, the generators of $\mathfrak{s u}(2)$ satisfies,

$$
\left[L^{j}, L^{k}\right]=i \epsilon^{j k r} L^{r}
$$

Now for convenience, we choose to work in the well known basis which are given by the Pauli matrices (equation 2.5). So letting

$$
L^{j}=\frac{1}{2} \sigma^{j} \quad, \text { for } j=1,2,3
$$


Thus, we arrive at:

$$
S U(2)=\left\{e^{\alpha^{j} L^{j}}=e^{\frac{i}{2} \sigma^{j}}, \text { for } j=1,2,3\right\}
$$

Finally, SU(3) is the special unitary group of order 3. This group has a total of 8 generators. Once again, we can use the most familiar generators which are given by the Gell-Mann matrices $\left(\lambda_{i}\right)$ that are defined as follow:

$$
\begin{gathered}
T_{i}=\frac{\lambda_{i}}{2} \quad, \text { for } i=1,2, \ldots, 8 \\
\lambda_{1}=\left(\begin{array}{lll}
0 & 1 & 0 \\
1 & 0 & 0 \\
0 & 0 & 0
\end{array}\right), \quad \lambda_{2}=\left(\begin{array}{lll}
0 & -i & 0 \\
i & 0 & 0 \\
0 & 0 & 0
\end{array}\right), \quad \lambda_{3}=\left(\begin{array}{lll}
1 & 0 & 0 \\
0 & -1 & 0 \\
0 & 0 & 0
\end{array}\right) \\
\lambda_{4}=\left(\begin{array}{lll}
0 & 0 & 1 \\
0 & 0 & 0 \\
1 & 0 & 0
\end{array}\right), \quad \lambda_{5}=\left(\begin{array}{lll}
0 & 0 & -i \\
0 & 0 & 0 \\
i & 0 & 0
\end{array}\right), \quad \lambda_{6}=\left(\begin{array}{lll}
0 & 0 & 0 \\
0 & 0 & 1 \\
0 & 1 & 0
\end{array}\right) \\
\lambda_{7}=\left(\begin{array}{lll}
0 & 0 & 0 \\
0 & 0 & -i \\
0 & i & 0
\end{array}\right), \lambda_{8}=\frac{1}{\sqrt{3}}\left(\begin{array}{ccc}
1 & 0 & 0 \\
0 & 1 & 0 \\
0 & 0 & -2
\end{array}\right)
\end{gathered}
$$


The Lie bracket for $\mathfrak{s u}(3)$ generators satisfies:

$$
\left[T_{j}, T_{k}\right]=i f_{j k r} T_{r}
$$

where $f_{j k r}$ is the anti-symmetric structure constant for $\mathrm{SU}(3)$, and it is given as:

$$
\begin{array}{r}
f_{123}=+1 \\
f_{458}=f_{678}=\frac{\sqrt{3}}{2} \\
f_{147}=-f_{156}=f_{257}=f_{345}=f_{246}=-f_{367}=\frac{1}{2}
\end{array}
$$

Thus, the group $\mathrm{SU}(3)$ is composed as:

$$
S U(3)=\left\{e^{\beta_{j} T_{j}}=e^{\frac{i}{2} \lambda_{j}} \quad, \quad \text { for } j=1,2, \ldots, 8\right\}
$$

\section{A.3 Rotation group}

Rotations are set of coordinate transformations that preserve the length and angles between vectors in space $\mathbf{R}^{n}$. It is easy to imagine rotation in two dimension (2D) Euclidean space $\mathbf{R}^{2}$, which in fact forms the $\mathrm{SO}(2)$ group (which is isomorphic to $\mathrm{U}(1))$. So for $2 \mathrm{D}$ rotation as shown in figure A.1, we can write:

$$
\left(\begin{array}{l}
x^{\prime} \\
y^{\prime}
\end{array}\right)=\left(\begin{array}{cc}
\cos (\theta) & \sin (\theta) \\
-\sin (\theta) & \cos (\theta)
\end{array}\right)\left(\begin{array}{l}
x \\
y
\end{array}\right)
$$




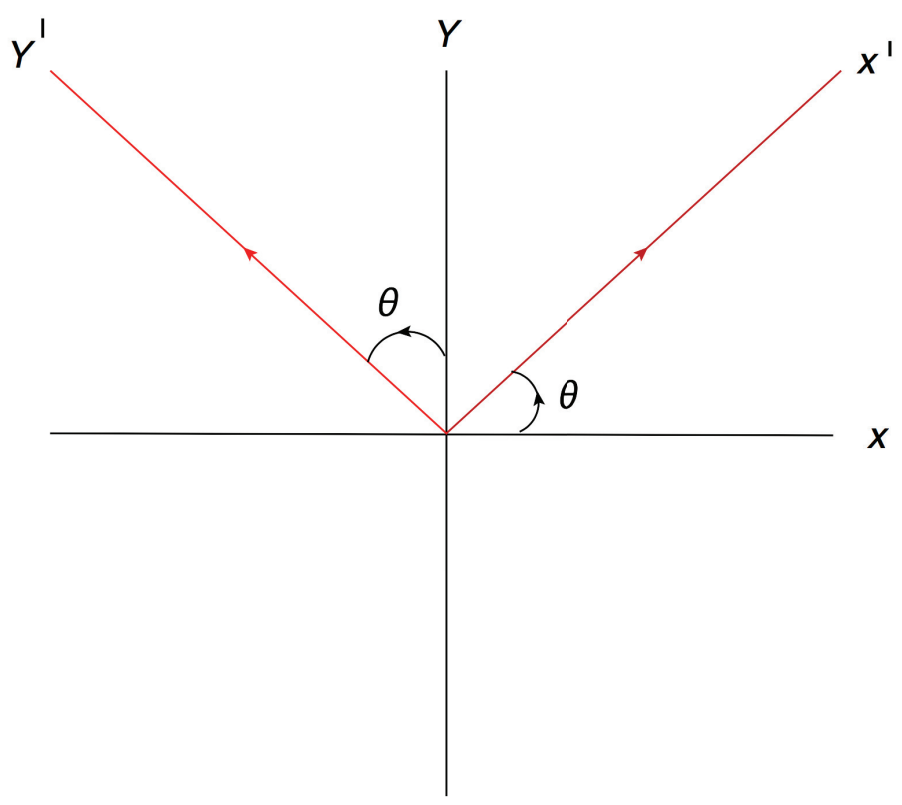

Figure A.1: A simple rotation in the xy-plane by an angle $\theta$ counterclockwise.

So a general rotation in two dimension $\mathcal{R}(\theta): R^{2} \longrightarrow R^{2}$ is given by:

$$
\mathcal{R}(\theta)=\left(\begin{array}{cc}
\cos (\theta) & \sin (\theta) \\
-\sin (\theta) & \cos (\theta)
\end{array}\right)
$$

Which satisfies $\mathcal{R}(\theta) \mathcal{R}(\theta)^{T}=\mathcal{R}(\theta)^{T} \mathcal{R}(\theta)=\mathbb{1}$ and $\operatorname{det}\{\mathcal{R}(\theta)\}=1$. Thus it forms the special orthogonal group of order $2(\mathrm{SO}(2))$.

Then going into three dimensional space, rotations are linear transformations of the vector $\mathbf{x}=\left(x_{1}, x_{2}, x_{3}\right)$

$$
x_{i}^{\prime}=\sum_{j} R_{i j} x_{j}
$$


Which leaves the inner product of the vector invariant:

$$
\begin{aligned}
\mathbf{x} \cdot \mathbf{x} & =\mathbf{x}^{\prime} \cdot \mathbf{x}^{\prime} \\
\sum_{j} x_{j}^{2} & =\sum_{i} x_{i}^{\prime 2}=\sum_{i j k} R_{i j} R_{i k} x_{j} x_{k} \Longrightarrow R_{i j} R_{i k}=\delta_{j k}
\end{aligned}
$$

So from that, we see that in terms of matrices the corresponding relations are:

$$
R R=\mathbb{I} \Longrightarrow \operatorname{det}\{R\}= \pm 1
$$

Therefore, the three dimensional rotations form the $\mathrm{SO}(3)$ group. For three dimensions one can do a rotation around an axis by angle $\theta$ and come up with the following rotation matrices:

$$
\begin{aligned}
\mathcal{R}_{x}=\left(\begin{array}{ccc}
1 & 0 & 0 \\
0 & \cos (\theta) & \sin (\theta) \\
0 & -\sin (\theta) & \cos (\theta)
\end{array}\right) \quad \mathcal{R}_{y}=\left(\begin{array}{cccc}
\cos (\theta) & 0 & -\sin (\theta) \\
0 & 1 & 0 \\
\sin (\theta) & 0 & \cos (\theta)
\end{array}\right) \\
\mathcal{R}_{z}=\left(\begin{array}{ccc}
\cos (\theta) & \sin (\theta) & 0 \\
-\sin (\theta) & \cos (\theta) & 0 \\
0 & 0 & 1
\end{array}\right)
\end{aligned}
$$

As before, it can be shown that these matrices satisfies $\mathcal{R}_{i} \mathcal{R}_{i}^{T}=\mathcal{R}_{i}^{T} \mathcal{R}_{i}=\mathbb{1}$ and $\operatorname{det} \mathcal{R}_{i}=1$ for $\mathrm{i}=\mathrm{x}, \mathrm{y}, \mathrm{z}$.

The Lie theory is all about continuous symmetries, so an element of the group 
close to the the identity can be written as:

$$
g(\epsilon)=\mathbb{I}+\epsilon X, \quad \text { where } \mathrm{X} \text { is the generator }
$$

We write the infinitesimal number as $\epsilon=\frac{\beta}{N}$, where $\beta$ is some finite transformation and $\mathrm{N}$ is a large number. Then in order to obtain a finite transformation from repeating these infinitesimal ones, we need to take $N \rightarrow \infty$. Thus

$$
\begin{aligned}
& f(\beta)=\lim _{N \rightarrow \infty}\left(\mathbb{I}+\frac{\beta}{N} X\right)^{N}=e^{\beta X} \quad \text { Taylor expanding this: } \\
& f(\beta)=\left.\sum_{m=0}^{\infty} \frac{\mathrm{d}^{m} f}{\mathrm{~d} \beta^{m}}\right|_{\beta=0} \beta^{m}=\mathbb{I}+\left.\beta \frac{\mathrm{d} f}{\mathrm{~d} \beta}\right|_{\beta=0}+\left.\beta^{2} \frac{\mathrm{d}^{2} f}{\mathrm{~d} \beta^{2}}\right|_{\beta=0}+\ldots
\end{aligned}
$$

Hence the generators are:

$$
X=\left.\frac{\mathrm{d} f}{\mathrm{~d} \beta}\right|_{\beta=0}
$$

Now in physics, we conventionally put an extra "i" in the generators to make them Hermitian. So the generators becomes:

$$
X=\left.i \frac{\mathrm{d} f}{\mathrm{~d} \beta}\right|_{\beta=0}
$$

We can apply this to the three dimensional rotations by using equation A.25, which 
gives generators as:

$$
\begin{aligned}
& J_{1}=\left.i \frac{\mathrm{d} \mathcal{R}_{x}}{\mathrm{~d} \theta}\right|_{\theta=0}=i\left(\begin{array}{ccc}
0 & 0 & 0 \\
0 & 0 & 1 \\
0 & -1 & 0
\end{array}\right) \\
& J_{2}=\left.i \frac{\mathrm{d} \mathcal{R}_{y}}{\mathrm{~d} \theta}\right|_{\theta=0}=i\left(\begin{array}{ccc}
0 & 0 & -1 \\
0 & 0 & 0 \\
1 & 0 & 0
\end{array}\right) \\
& J_{3}=\left.i \frac{\mathrm{d} \mathcal{R}_{z}}{\mathrm{~d} \theta}\right|_{\theta=0}=i\left(\begin{array}{ccc}
0 & 1 & 0 \\
-1 & 0 & 0 \\
0 & 0 & 0
\end{array}\right)
\end{aligned}
$$

Using the above generators, one can show explicitly that they satisfy:

$$
\left[J_{a}, J_{b}\right]=i \epsilon_{a b c} J_{c}
$$

Thus a finite rotation about a direction $\mathbf{n}$ is:

$$
\mathcal{R}=e^{-\theta \mathbf{J} \cdot \mathbf{n}}
$$

Finally, a finite rotation about a fixed axis in the four-vector space is represented by: 


$$
\left[\begin{array}{c:c} 
& 0 \\
1 & 0 \\
\hdashline 0 & \mathcal{R}_{i}
\end{array}\right]
$$

Where $\mathcal{R}_{i}$ is the element of the three dimensional rotation group.

\section{A.4 Lorentz Invariance}

Lorentz transformation are linear spacetime coordinate transformation connecting any two inertial frames of reference. Such transformations are defined as,

$$
x_{\mu}^{\prime}=\Lambda_{\mu}^{\nu} x_{\nu}
$$

with the condition

$$
\sum_{\mu} x_{\mu}^{\prime} \cdot x^{\mu \prime}=x^{\prime 2}=\sum_{\mu} x_{\mu} \cdot x^{\mu}=x^{2}
$$

The spacetime components $\Lambda_{i}^{0}$ are the Lorentz boosts and $\Lambda_{j}^{i}$ are the three dimensional rotation of Euclidean space. Recall, the covariant four-vector is:

$$
\begin{aligned}
& x^{\mu}=(c t, x, y, z)=\left(x^{0}, x^{1}, x^{2}, x^{3}\right) \\
& x_{\mu}=g_{\mu \nu} x^{\nu}, \quad \text { where } g_{\mu \nu}=g^{\mu \nu} \text { is the matrix tensor }
\end{aligned}
$$


So the expression in equation A.31 gives:

$$
\begin{array}{r}
g_{\mu \nu} x^{\prime \nu} x^{\prime \mu}=g_{\mu \nu} \Lambda_{\rho}^{\nu} \Lambda_{\sigma}^{\mu} x^{\rho} x^{\sigma}=g_{\sigma \rho} x^{\rho} x^{\sigma} \\
\Longrightarrow \Lambda_{\sigma}^{\mu} g_{\mu \nu} \Lambda_{\rho}^{\nu}=g_{\sigma \rho}
\end{array}
$$

Which can be represented in terms of matrices as:

$$
\begin{aligned}
\Lambda^{T} g \Lambda & =g, \quad \text { which implies } \\
\operatorname{det}\left(\Lambda^{T} g \Lambda\right) & =(\operatorname{det} \Lambda)^{2}=1 \Longrightarrow \operatorname{det}(\Lambda)= \pm 1
\end{aligned}
$$

It is clear that the of set matrices $\Lambda$ form a group since they satisfy all the axioms of the group. The composition of the two transformations is again a Lorentz transformation, the identity matrix II is part of the set of matrices $\Lambda$, matrix multiplication is associative and equation A.34 ensures the existence of the inverse element $\Lambda^{-1}$. The pure Lorentz boost about an axis $x^{1}$ is given by [30]:

$$
L_{1}=\left(\begin{array}{cccc}
\gamma & -\beta \gamma & 0 & 0 \\
-\beta \gamma & \gamma & 0 & 0 \\
0 & 0 & 1 & 0 \\
0 & 0 & 0 & 1
\end{array}\right), \quad \text { where } \beta=\frac{v}{c}, \gamma=\left(1-\beta^{2}\right)^{-\frac{1}{2}}
$$

We can further rewrite the Lorentz boost by defining a new parameter 'w' such that $\cosh (w)=\gamma$. Then

$$
\cosh (w)=\gamma=\left(1-\beta^{2}\right)^{-\frac{1}{2}} \Longrightarrow \beta \gamma=\left(\gamma^{2}-1\right)^{\frac{1}{2}}=\sinh (w)
$$


Consequently,

$$
\begin{aligned}
L_{1} & =\left(\begin{array}{ccccc}
\cosh (w) & -\sinh (w) & 0 & 0 \\
-\sinh (w) & \cosh (w) & 0 & 0 \\
0 & 0 & 1 & 0 \\
0 & 0 & 0 & 1
\end{array}\right) \\
L_{2} & =\left(\begin{array}{ccccc}
\cosh (w) & 0 & -\sinh (w) & 0 \\
0 & 1 & 0 & 0 \\
-\sinh (w) & 0 & \cosh (w) & 0 \\
0 & 0 & 0 & 1
\end{array}\right) \\
L_{3} & =\left(\begin{array}{ccccc}
\cosh (w) & 0 & 0 & -\sinh (w) \\
0 & 1 & 0 & 0 & \\
0 & 0 & 1 & 0 & \\
-\sinh (w) & 0 & 0 & \cosh (w)
\end{array}\right)
\end{aligned}
$$


So the complete set of generators for the boost can be obtained using equation A.25:

$$
\begin{aligned}
& K_{1}=i\left(\begin{array}{cccc}
0 & -1 & 0 & 0 \\
-1 & 0 & 0 & 0 \\
0 & 0 & 0 & 0 \\
0 & 0 & 0 & 0
\end{array}\right) \\
& K_{2}=i\left(\begin{array}{cccc}
0 & 0 & -1 & 0 \\
0 & 0 & 0 & 0 \\
-1 & 0 & 0 & 0 \\
0 & 0 & 0 & 0
\end{array}\right) \\
& K_{3}=i\left(\begin{array}{cccc}
0 & 0 & 0 & -1 \\
0 & 0 & 0 & 0 \\
0 & 0 & 0 & 0 \\
-1 & 0 & 0 & 0
\end{array}\right)
\end{aligned}
$$

Then, a finite pure Lorentz boost transformation about a fixed axis $\alpha=\frac{\mathbf{v}}{|\mathbf{v}|}$ can be written as:

$$
L=e^{-w \mathbf{K} \cdot \hat{\alpha}}
$$


One can explicitly show that the above generators satisfy:

$$
\begin{aligned}
{\left[K_{a}, K_{b}\right] } & =i \epsilon_{a b c} J_{c} \\
{\left[J_{a}, K_{b}\right] } & =i \epsilon_{a b c} K_{c}, \quad \text { Also recall }\left[J_{a}, J_{b}\right]=i \epsilon_{a b c} J_{c}
\end{aligned}
$$

Together rotations and boosts form the proper orthochronous Lorentz group for $\Lambda_{0}^{0} \geq$ +1 and $\operatorname{det} \Lambda=+1$. To sum up, a general finite Lorentz transformation for this group is:

$$
\Lambda=e^{-(w \mathbf{K} \cdot \hat{\alpha}+\theta \mathbf{J} \cdot \mathbf{n})}
$$

\section{A.5 Basic Statistics}

In particle physics we are often dealing with a large of amount of data to be analyzed so we require a method to differentiate signal like events from background like. Most of the time, we are interested in how significant a particular observation is and when can we declare a discovery in favour of a new affect? Thus we resort to statistical techniques to answer some of these questions. Lets begin with a brief introduction to statistics. One of the goals of an experimentalist is to perform a statistical test to make a statement about how well the predicted probability stands in comparison to the observed data. Conventionally the default hypothesis under consideration is called the null hypothesis $H_{0}$, which one often tries to nullify and there is an opposing test represented by the alternative hypothesis $H_{a}$. Now in order to test an agreement between data and a given hypothesis, we need to a construct a selection criteria on which a decision for the hypothesis is based on. It is a called the test statistic.

In light of the binary decision from the test statistic, it is possible to incorrectly reject the null hypothesis when it is true. This is called a type I error and it is 
denoted by $\alpha$ (significance level) [31]. So for a model with a given probability density function (f), for a given test statistic (T), we have:

$$
\alpha=\int_{T_{d}}^{\infty} f\left(T \mid H_{0}\right) d T
$$

Where $T_{d}$ is the cut off value of the test statistic above which the null hypothesis is rejected. However, it is also possible to to accept the null hypothesis when it is not true. This referred to as a type II error and is denoted by $\beta$. Thus we have,

$$
\beta=\int_{-\infty}^{T_{d}} f\left(T \mid H_{a}\right) d T
$$

As mentioned before, one is more often interested in how compatible the null hypothesis is with the observed data. This test is known as the goodness of fit and the significance of the test is represented by the $P$-value. The $P$-value is essentially the probability of observing an event that is at least as contradictory as the one observed under the assumption that the null hypothesis is true [31]. Mathematically it is given as,

$$
P-\text { value }=P\left(n \geq n_{\text {observed }}\right)
$$

For a counting experiment, $\mathrm{n}$ would be the number of events. So we reject the null hypothesis, $H_{0}$, if $P-$ value $\leq \alpha$. In other words, $\mathrm{P}$-value is the minimum observed significance level at which the null hypothesis can be rejected.

Furthermore, we define the expectation value, $E(x)$, which is basically an average for a random variable distributed according to a probability distribution $f(x)$ and is 
defined as:

$$
E(x)=\mu \equiv \int_{-\infty}^{\infty} x f(x) d x
$$

This is also called the first algebraic moment and another related quantity that is often used is the second central moment,

$$
E\left[(x-E(x))^{2}\right]=V(x) \equiv \int_{-\infty}^{\infty}(x-\mu)^{2} f(x) d x=\sigma^{2}
$$

Where $\sigma$ is known as the standard deviation.

Beside the significance level $\alpha$, another term that is often used is the confidence level $(100 \%(1-\alpha))$ which is essentially the probability for the occurrence of the true value of the statistics within the obtained interval using the applied method. In physics our concern lies with the confidence level of the signal but in actual experiments our signal is accompanied by background. So we have to deal with the inevitable background and possible error associated with it. We use the so called modified frequentist confidence level, which is defined as [28]:

$$
C L_{s}=\frac{C L_{s+b}}{C L_{b}}
$$

Where $C L_{s+b}$ is the confidence level for excluding a possible signal and background hypothesis,

$$
C L_{s+b}=P_{s+b}\left(X \leq X_{o b s}\right)
$$

$C L_{b}$ is the probability for background only hypothesis of observing events less than 
or equal to the events observed.

$$
C L_{b}=P_{b}\left(X \leq X_{o b s}\right)
$$

Then the confidence level is $C L=\left(1-C L_{s}\right) \times 100 \%$. In actual experiments we are dealing with systematic uncertainties associated with background and signal, so we need to include its effects on the confidence intervals. These systematic uncertainties are encoded in variables called nuisance parameters which modifies the confidence interval but they are not the primary observable of interest to us. So for a counting experiment, with the gaussian error on the background, the confidence level are constructed as follow:

$$
\begin{aligned}
C L_{b}\left(n, b, \sigma_{b}\right) & =\frac{\sum_{i=0}^{i=n} \int_{0}^{\infty} \frac{1}{\sqrt{2 \pi} \sigma_{b}} e^{-\frac{\left(b-b^{\prime}\right)^{2}}{2 \sigma^{2}} \frac{b^{\prime} e^{-b^{\prime}}}{i !}} \mathrm{d} b^{\prime}}{\int_{0}^{\infty} \frac{1}{\sqrt{2 \pi} \sigma_{b}} e^{-\frac{\left(b-b^{\prime}\right)^{2}}{2 \sigma^{2}}} \mathrm{~d} b^{\prime}} \\
C L_{s+b}\left(n, s, b, \sigma_{b}\right) & =\frac{\sum_{i=0}^{i=n} \int_{0}^{\infty} \frac{1}{\sqrt{2 \pi} \sigma_{b}} e^{-\frac{\left(b-b^{\prime}\right)^{2}}{2 \sigma^{2}} \frac{\left(b^{\prime}+s\right)^{i} e^{-\left(b^{\prime}+s\right)}}{i !} \mathrm{d} b^{\prime}}}{\int_{0}^{\infty} \frac{1}{\sqrt{2 \pi} \sigma_{b}} e^{-\frac{\left(b-b^{\prime}\right)^{2}}{2 \sigma^{2}}} \mathrm{~d} b^{\prime}}
\end{aligned}
$$




\section{Appendix B}

\section{Physics Tools}

\section{B.1 Solution to the Dirac equation}

Lets define some notation before we solve the Dirac equation:

$$
\sigma^{\mu} \equiv(1, \boldsymbol{\sigma}), \bar{\sigma}^{\mu} \equiv(1,-\boldsymbol{\sigma})
$$

so,

$$
\gamma^{\mu}=\left(\begin{array}{cc}
0 & \sigma^{\mu} \\
\bar{\sigma}^{\mu} & 0
\end{array}\right)
$$

Note that

$$
(p \cdot \sigma)(p \cdot \bar{\sigma})=p_{0}^{2}-p_{i} p_{j} \sigma^{i} \sigma^{j}=p_{0}^{2}-p_{i} p_{j} \delta^{i j}=p_{\mu} p^{\mu}=m^{2}
$$

The Dirac equation is:

$$
\left(i \gamma^{\mu} \partial_{\mu}-m\right) \psi(x)=0
$$


The Dirac field can be written as a linear combination of plane waves:

$$
\psi(x)=u(p) e^{-i p \cdot x}, \quad \text { such that } p^{2}=m^{2}
$$

Plugging this into the Dirac equation B.4 becomes,

$$
\left(\gamma^{\mu} p_{\mu}-m\right) u(p)=\left(\begin{array}{cc}
-m & p_{\mu} \sigma^{\mu} \\
p_{\mu} \bar{\sigma}^{\mu} & -m
\end{array}\right) u(p)=0
$$

Rewriting the four component spinor as $u(p)=\left(\begin{array}{l}\xi_{1} \\ \xi_{2}\end{array}\right)$, then equation B.6 gives:

$$
p \cdot \sigma \xi_{2}=m \xi_{1}, \quad p \cdot \bar{\sigma} \xi_{1}=m \xi_{2}
$$

Where $\xi_{1}$ and $\xi_{2}$ are two component spinors.

Lets choose,

$$
\xi_{1}=\frac{1}{m}(p \cdot \sigma)(\sqrt{p \cdot \bar{\sigma}}) \xi=(\sqrt{p \cdot \sigma}) \xi
$$

Hence the second expression in equation B.7 implies,

$$
\xi_{2}=(\sqrt{p \cdot \bar{\sigma}}) \xi
$$


Consequently, the solution is:

$$
u(p)^{s}=\left(\begin{array}{c}
\sqrt{p \cdot \sigma} \xi^{s} \\
\sqrt{p \cdot \bar{\sigma}} \xi^{s}
\end{array}\right), \quad s=1,2
$$

Now in the beginning we only considered the positive frequency scenario $\left(p^{0}>0\right)$; however, there is second case where the frequency is negative. Then again applying the same steps and expanding the Dirac field in terms of the plane waves,

$$
\psi(x)=v(p) e^{+i p \cdot x}
$$

which in turn gives,

$$
\left(\gamma^{\mu} p_{\mu}+m\right) v(p)=\left(\begin{array}{cc}
m & p_{\mu} \sigma^{\mu} \\
p_{\mu} \bar{\sigma}^{\mu} & m
\end{array}\right) v(p)=0
$$

Thus by similar method as before, the solution is:

$$
v(p)^{s}=\left(\begin{array}{c}
\sqrt{p \cdot \sigma} \eta^{s} \\
-\sqrt{p \cdot \bar{\sigma}} \eta^{s}
\end{array}\right), \quad s=1,2
$$

\section{B.1.1 Spin Sums}

By convention, we normalize the two component spinors as $\xi^{\dagger} \xi=\eta^{\dagger} \eta=1$. Using the solutions for the four-component spinors and doing the sum over the spins, the 
Lorentz invariant quantity gives:

$$
\begin{aligned}
& \sum_{s=1,2} u^{s}(p) \bar{u}^{s}(p)=\sum_{s=1,2}\left(\begin{array}{c}
\sqrt{p \cdot \sigma} \xi^{s} \\
\sqrt{p \cdot \bar{\sigma}} \xi^{s}
\end{array}\right)\left(\xi^{s \dagger} \sqrt{p \cdot \bar{\sigma}}, \xi^{s \dagger} \sqrt{p \cdot \sigma}\right) \\
& =\left(\begin{array}{ll}
\sqrt{p \cdot \sigma} \sqrt{p \cdot \bar{\sigma}} & \sqrt{p \cdot \sigma} \sqrt{p \cdot \sigma} \\
\sqrt{p \cdot \bar{\sigma}} \sqrt{p \cdot \bar{\sigma}} & \sqrt{p \cdot \bar{\sigma}} \sqrt{p \cdot \sigma}
\end{array}\right) \\
& =\left(\begin{array}{cc}
m & p \cdot \sigma \\
p \cdot \bar{\sigma} & m
\end{array}\right)=\gamma^{\mu} p_{\mu}+m
\end{aligned}
$$

Similarly we get,

$$
\sum_{s=1,2} v^{s}(p) \bar{v}^{s}(p)=\left(\begin{array}{cc}
-m & p \cdot \sigma \\
p \cdot \bar{\sigma} & -m
\end{array}\right)=\gamma^{\mu} p_{\mu}-m
$$

\section{B.2 Traces}

Trace is defined as the sum of the main diagonal elements of a $n \times n$ square matrix, so the trace of a matrix $M$ is:

$$
\operatorname{tr}(M)=\sum_{i=1}^{n} m_{i i}=m_{11}+m_{22}+\ldots+m_{n n}
$$

Some of the basic trace properties are as follow:

1. $\operatorname{tr}(A+B)=\operatorname{tr}(A)+\operatorname{tr}(B)$

2. $\operatorname{tr}\left(c_{1} A+c_{2} B\right)=c_{1} \operatorname{tr}(A)+c_{2} \operatorname{tr}(B)$ 
3. $\operatorname{tr}(A B C)=\operatorname{tr}(B C A)=\operatorname{tr}(C A B), \quad$ cyclic permutation

The main interest in physics for traces lies in the Dirac gamma matrices $(4 \times 4)$. So lets begin with some of their identities.

1. $\operatorname{tr}(\mathbb{1})=4$

2. $\operatorname{tr} \gamma^{5}=0$

3. $\operatorname{tr} \gamma^{\mu}=\operatorname{tr}\left(\begin{array}{cc}0 & \sigma^{\mu} \\ \bar{\sigma}^{\mu} & 0\end{array}\right)=0$

Proof. An abstract proof that can be generalized to any odd number of $\gamma$ matrices:

$$
\begin{aligned}
\operatorname{tr} \gamma^{\mu} & =\operatorname{tr}\left(\gamma^{5} \gamma^{5} \gamma^{\mu}\right), \quad \text { Because }\left(\gamma^{5}\right)^{2}=1 \\
& =-\operatorname{tr}\left(\gamma^{5} \gamma^{\mu} \gamma^{5}\right), \quad \text { Because }\left\{\gamma^{\mu}, \gamma^{5}\right\}=0 \\
& =-\operatorname{tr}\left(\gamma^{5} \gamma^{5} \gamma^{\mu}\right), \quad \text { Because of cyclic permutation } \\
& =-\operatorname{tr}\left(\gamma^{\mu}\right)
\end{aligned}
$$

$\therefore$ The trace of $\gamma^{\mu}$ is zero since it is equal to minus itself.

We can generalize this to any $n$ odd $\gamma$ matrices. The only thing that changes in the above proof is that in the second line we would instead get a factor of $(-1)^{n}=-1$ from moving one of the $\gamma^{5}$ all the way to the right. The rest of the steps are exactly as before, so the trace of an odd n-gamma matrices must 
vanish.

$$
\operatorname{tr}\left(\text { Odd number of } \gamma^{\prime} \mathrm{s}\right)=0
$$

4. $\operatorname{tr}\left(\gamma^{\mu} \gamma^{\nu}\right)=4 g^{\mu \nu}$

Proof.

$$
\begin{aligned}
\operatorname{tr}\left(\gamma^{\mu} \gamma^{\nu}\right) & =\operatorname{tr}\left(2 g^{\mu \nu} \mathbb{I}-\gamma^{\nu} \gamma^{\mu}\right)=8 g^{\mu \nu}-\operatorname{tr}\left(\gamma^{\mu} \gamma^{\nu}\right), \text { Cyclical permutation } \\
& =4 g^{\mu \nu}
\end{aligned}
$$

A similar procedure can be applied to four or more $\gamma$ matrices.

5. $\operatorname{tr}\left(\gamma^{\mu} \gamma^{\nu} \gamma^{\rho} \gamma^{\sigma}\right)=4\left(g^{\mu \nu} g^{\rho \sigma}-g^{\mu \rho} g^{\nu \sigma}+g^{\mu \sigma} g^{\nu \rho}\right)$

6. $\operatorname{tr}\left(\gamma^{\mu} \gamma_{\mu}\right)=16$

Proof.

$$
\begin{aligned}
\operatorname{tr}\left(\gamma^{\mu} \gamma_{\mu}\right) & =\operatorname{tr}\left(g_{\mu \nu} \gamma^{\mu} \gamma^{\nu}\right)=\operatorname{tr}\left(\frac{1}{2} g_{\mu \nu}\left\{\gamma^{\mu}, \gamma^{\nu}\right\}\right)= \\
& =\operatorname{tr}\left(g_{\mu \nu} g^{\mu \nu} \mathbb{I}\right)=\operatorname{tr}(4 \mathbb{I})=16
\end{aligned}
$$

7. $\operatorname{tr}\left(\gamma^{\mu} \gamma^{\nu} \gamma^{5}\right)=0$

Proof. Using the fact that $\gamma^{\sigma} \gamma^{\sigma}=g^{\sigma \sigma}$ and $\left\{\gamma^{\mu}, \gamma^{\sigma}\right\}=0$ for $\sigma \neq \mu$. So we take 
$\sigma \neq \mu, \nu$ and proceeds as follows:

$$
\begin{aligned}
\operatorname{tr}\left(\gamma^{\mu} \gamma^{\nu} \gamma^{5}\right) & =\operatorname{tr}\left(g_{\sigma \sigma} \gamma^{\sigma} \gamma^{\sigma} \gamma^{\mu} \gamma^{\nu} \gamma^{5}\right) \\
& =\operatorname{tr}\left(g_{\sigma \sigma}(-1)^{2} \gamma^{\sigma} \gamma^{\mu} \gamma^{\nu} \gamma^{\sigma} \gamma^{5}\right), \quad \text { Because }\left\{\gamma^{\sigma}, \gamma^{5}\right\}=0 \\
& =\operatorname{tr}\left(g_{\sigma \sigma}(-1)^{3} \gamma^{\sigma} \gamma^{\mu} \gamma^{\nu} \gamma^{5} \gamma^{\sigma}\right), \quad \text { Because of cyclic permutation } \\
& =(-1)^{3} \operatorname{tr}\left(g_{\sigma \sigma} \gamma^{\sigma} \gamma^{\sigma} \gamma^{\mu} \gamma^{\nu} \gamma^{5}\right) \\
& =-\operatorname{tr}\left(\gamma^{\mu} \gamma^{\nu} \gamma^{5}\right)
\end{aligned}
$$

Finally, lets show some useful contraction identities.
a. $\gamma^{\mu} \gamma_{\mu}=4 \mathbb{I}$
b. $\gamma^{\mu} \gamma^{\nu} \gamma_{\mu}=-2 \gamma^{\nu}$
c. $\gamma^{\mu} \gamma^{\nu} \gamma^{\rho} \gamma_{\mu}=4 g^{\nu \rho}$
d. $\gamma^{\mu} \gamma^{\nu} \gamma^{\rho} \gamma^{\sigma} \gamma_{\mu}=-2 \gamma^{\sigma} \gamma^{\rho} \gamma^{\nu}$

We have already shown $a$, lets prove $b$ and the rest can be obtained by a similar method.

Proof.

$$
\gamma^{\mu} \gamma^{\nu} \gamma_{\mu}=\left(2 g^{\mu \nu} \mathbb{I}-\gamma^{\nu} \gamma^{\mu}\right) \gamma_{\mu}=2 \gamma^{\nu}-4 \gamma^{\nu}=-2 \gamma^{\nu}
$$




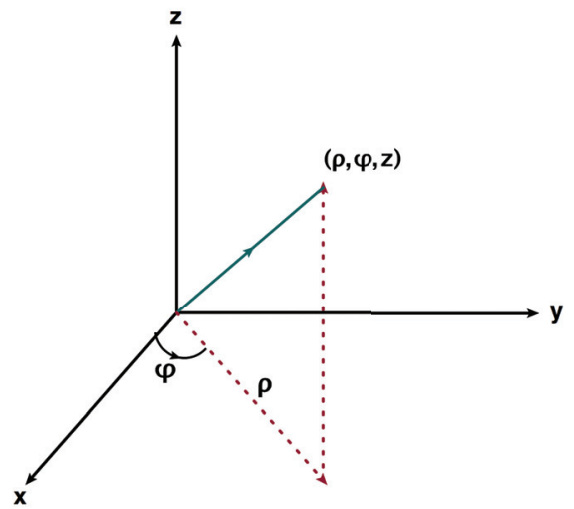

(a) General cylindrical coordinates

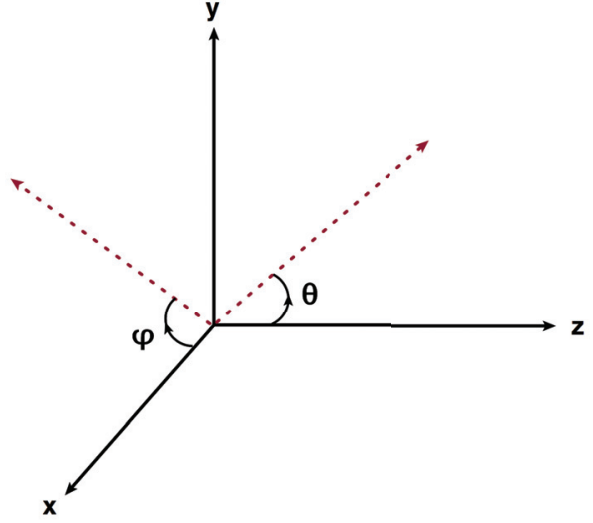

(b) LHC coordinate system

Figure B.1: A typical coordinate system used in colliders.

\section{B.3 CKM Matrix}

The magnitudes of elements of the famous Cabibbo-Kobayashi-Maskawa (CKM) matrix that gives the mixing between quarks are [11]:

$$
V_{C K M}=\left(\begin{array}{rrr}
0.97434_{-0.00012}^{+0.00011} & 0.22506 \pm 0.00050 & 0.00357 \pm 0.00015 \\
0.22492 \pm 0.00050 & 0.97351 \pm 0.00013 & 0.0411 \pm 0.0013 \\
0.00875_{-0.00033}^{+0.00032} & 0.0403 \pm 0.0013 & 0.99915 \pm 0.00005
\end{array}\right)
$$

\section{B.4 Colliders Coordinate System}

The coordinate system used at the LHC is different from the usual cartesian, spherical, or cylindrical coordinate system. The ATLAS and CMS detectors are cylindrical so a convenient system would be to start with cylindrical coordinates $(\rho, \phi, z)$ as shown in figure B.1.

At the LHC, the origin of the coordinate system lies at the interaction point and the $\mathrm{z}$-axis lies along the beam line. So the transverse plane is perpendicular to the 
beam and is defined by the $\mathrm{x}-\mathrm{y}$ coordinates. The positive $\mathrm{x}$-axis points toward the center of the LHC ring and the positive y-axis points upward toward the surface. The azimuthal angle $\phi$ is measured from the $\mathrm{x}$-axis and the polar angle is measured from the beam line as demonstrated in figure B.1. But the polar angle $\theta$ is replaced by pseudo - rapidity that is defined as:

$$
\eta \equiv-\ln \left[\tan \left(\frac{\theta}{2}\right)\right]
$$

Which can be rewritten in terms of the total 3-momentum $|\boldsymbol{p}|$ and the momentum along the beam direction $p_{z}$. So using the double angle trigonometric identities,

$$
\begin{aligned}
& \cos (\theta)=2 \cos ^{2}\left(\frac{\theta}{2}\right)-1 \Longrightarrow \cos ^{2}\left(\frac{\theta}{2}\right)=\frac{1}{2}[\cos (\theta)+1]=\frac{1}{2}\left[\frac{p_{z}}{|\boldsymbol{p}|}+1\right] \\
& \cos (\theta)=1+2 \sin ^{2}\left(\frac{\theta}{2}\right) \Longrightarrow \sin ^{2}\left(\frac{\theta}{2}\right)=\frac{1}{2}[\cos (\theta)-1]=\frac{1}{2}\left[\frac{p_{z}}{|\boldsymbol{p}|}-1\right]
\end{aligned}
$$

Then the pseudo - rapidity becomes,

$$
\begin{aligned}
& \eta=-\ln \left[\tan \left(\frac{\theta}{2}\right)\right]=\frac{1}{2} \ln \left[\frac{\cos ^{2}\left(\frac{\theta}{2}\right)}{\sin ^{2}\left(\frac{\theta}{2}\right)}\right]=\frac{1}{2} \ln \left\{\frac{\frac{1}{2}\left[\frac{p_{z}}{|p|}+1\right]}{\frac{1}{2}\left[\frac{p_{z}}{|\boldsymbol{p}|}-1\right]}\right\} \\
& \eta=\frac{1}{2} \ln \left[\frac{|\boldsymbol{p}|+p_{z}}{|\boldsymbol{p}|-p_{z}}\right]
\end{aligned}
$$

Furthermore we define another useful quantity called the rapidity that is widely used in the colliders,

$$
y \equiv \frac{1}{2} \ln \left[\frac{E+p_{z}}{E-p_{z}}\right]
$$

and one notices that in the highly relativistic limit $|\boldsymbol{p}| \gg m$, then $y \approx \eta$. Rapidity is a useful quantity for colliders because the differences in rapidity are Lorentz invariant. 
Consider a boost along the z-axis,

$$
\begin{aligned}
y \stackrel{\text { Lorentz boost }}{\longrightarrow} y^{\prime} & =\frac{1}{2} \ln \left[\frac{E^{\prime}+p_{z}^{\prime}}{E^{\prime}-p_{z}^{\prime}}\right]=\frac{1}{2} \ln \left[\frac{\gamma\left(E-\beta p_{z}\right)+\gamma\left(p_{z}-\beta E\right)}{\gamma\left(E-\beta p_{z}\right)-\gamma\left(p_{z}-\beta E\right)}\right] \\
& =\frac{1}{2} \ln \left[\frac{\left(E+p_{z}\right)(1-\beta)}{\left(E-p_{z}\right)(1+\beta)}\right]
\end{aligned}
$$

Then the difference in rapidities for two particles in the boosted frame is:

$$
\begin{aligned}
\Delta y=y_{2}-y_{1} \stackrel{\text { Lorentz boost }}{\longrightarrow} \Delta y^{\prime} & =\frac{1}{2} \ln \left[\frac{\left(E_{2}+p_{z, 2}\right)(1-\beta)}{\left(E_{2}-p_{z, 2}\right)(1+\beta)}\right]-\frac{1}{2} \ln \left[\frac{\left(E_{1}+p_{z, 1}\right)(1-\beta)}{\left(E_{1}-p_{z, 1}\right)(1+\beta)}\right] \\
& =\frac{1}{2} \ln \left[\frac{\left(E_{2}+p_{z, 2}\right)\left(E_{1}-p_{z, 1}\right)}{\left(E_{2}-p_{z, 2}\right)\left(E_{1}+p_{z, 1}\right)}\right]=\Delta y
\end{aligned}
$$

Consequently one can define a Lorentz invariant angular separation between two particles for boost along the z-axis as,

$$
\Delta R=\sqrt{(\Delta \phi)^{2}+(\Delta y)^{2}}
$$

Finally, most often in hadron colliders the momentum measurements are done in the transverse plane. So instead of using the cartesian momenta $\left(p_{x}, p_{y}, p_{z}\right)$, we need the transverse momentum $p_{T}$. As such their corresponding relationships are:

$$
\begin{aligned}
& p_{T}=\sqrt{\left(p_{x}\right)^{2}+\left(p_{y}\right)^{2}} \\
& p_{x}=p_{T} \cos \phi, \quad p_{y}=p_{T} \cos \phi, \quad p_{z}=p_{T} \sinh \eta
\end{aligned}
$$


To obtain $p_{z}$ in terms of $p_{T}$ and $\eta$ using equation B.26;

$$
\begin{aligned}
\eta=\frac{1}{2} \ln \left[\frac{|\mathbf{p}|+p_{z}}{|\mathbf{p}|-p_{z}}\right] \Longrightarrow e^{\eta}-e^{-\eta} & =\left[\frac{|\mathbf{p}|+p_{z}}{|\mathbf{p}|-p_{z}}\right]^{\frac{1}{2}}-\left[\frac{|\mathbf{p}|-p_{z}}{|\mathbf{p}|+p_{z}}\right]^{\frac{1}{2}} \\
2 \sinh \eta & =\frac{\mid\left(\mathbf{p} \mid+p_{z}\right)-\left(\mathbf{p}-p_{z}\right)}{\left[\left(|\mathbf{p}|+p_{z}\right)\left(|\mathbf{p}|-p_{z}\right)\right]^{\frac{1}{2}}} \\
2 \sinh \eta & =\frac{2 p_{z}}{\sqrt{|\mathbf{p}|^{2}-\left(p_{z}\right)^{2}}}=\frac{2 p_{z}}{p_{T}} \\
\therefore p_{z} & =p_{T} \sinh \eta
\end{aligned}
$$

\section{B.5 cross section}

The infinitesimal cross section for a process where two particles collide and generate a certain number of final state particles is given by [9]:

$$
d \sigma=\frac{1}{2 E_{A} 2 E_{B}\left|v_{a}-v_{b}\right|}\left(\prod_{f} \int \frac{d^{3} p_{f}}{(2 \pi)^{3} 2 E_{f}}\right) \times\left|\mathcal{M}\left(p_{A}, p_{B} \longrightarrow p_{f}\right)\right|^{2}(2 \pi)^{4} \delta^{(4)}\left(P-\sum p_{f}\right)
$$

with $\mathrm{P}=$ Total initial 4-momentum ,

$p_{A, B}=$ The 4-momentum of initial states A,B

$p_{f}=$ The 4-momentum of the final state particles

$\left|v_{A}-v_{B}\right|=$ The relative velocity difference in the beam (where $\vec{v}=\frac{\vec{p}}{E}$ )

Where $\mathcal{M}$ is known as the invariant matrix element which can be determined by using the Feynman rules for the given process. The invariant matrix element $\mathcal{M}$ contains all the dynamics of the interactions. To evaluate $\mathcal{M}$, one needs to apply the Feynman rules which are dependent on the interaction Lagrangian and the type of particles under consideration. To make the idea of Feynman rules a little more explicit, one can examine the example for the Yukawa theory. Starting with the 
Lagrangian,

$$
\begin{aligned}
\mathcal{L}_{\text {Yukawa }} & =i \bar{\psi} \phi_{\mu} \psi-m_{\psi} \bar{\psi} \psi+\frac{1}{2} \partial_{\mu} \phi \partial^{\mu} \phi-\frac{1}{2} m_{\phi}^{2} \phi^{2}-\lambda \bar{\psi} \psi \phi \\
& =\mathcal{L}_{\text {Dirac }}+\mathcal{L}_{\text {Klein-Gordon }}-\lambda \bar{\psi} \psi \phi \\
\Longrightarrow \mathcal{H} & =\bar{\psi}\left(m_{\psi}-i \gamma^{j} \partial_{j}\right) \psi+\frac{1}{2}\left[\dot{\phi}^{2}+\frac{1}{2}(\nabla \phi)^{2}+m_{\phi}^{2} \phi^{2}\right]+\lambda \bar{\psi} \psi \phi \\
& =\mathcal{H}_{\text {Dirac }}+\mathcal{H}_{\text {Klein-Gordon }}+\mathcal{H}_{\text {interaction }}
\end{aligned}
$$

Consequently, $H_{\text {interaction }}=\int d^{3} x \mathcal{H}=-\int d^{3} x \mathcal{L}_{\text {interaction }}=\int d^{3} x \lambda \bar{\psi} \psi \phi$

From this Hamiltonian one can derive the following Feynman rules [7]:

a. Propagators:

$$
\overrightarrow{\phi(x) \phi}(y)=------=\frac{i}{q^{2}-m_{\phi}^{2}+i \epsilon}
$$

$$
\overrightarrow{\psi(x) \bar{\psi}}(y)=\longrightarrow \quad=\frac{i(q+m)}{q^{2}-m^{2}+i \epsilon}
$$

b. Vertices:

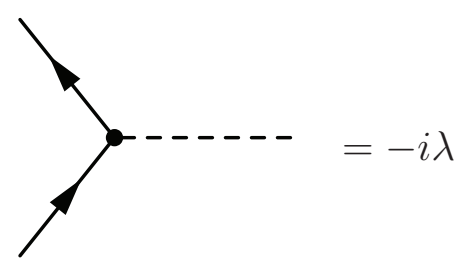

c. External leg contractions:

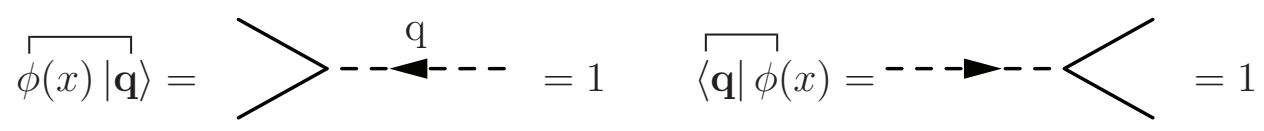



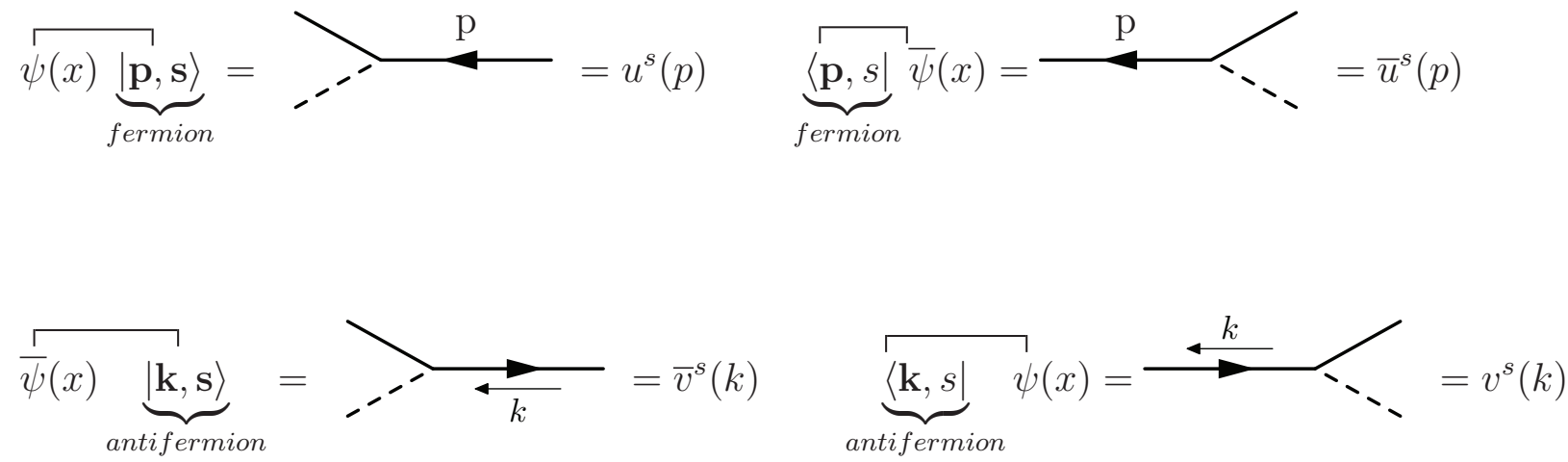

d. At each vertex, we impose four-momentum conservation

e. Complete the integration over each of the undetermined loop momenta

f. Determine the overall sign of the diagram

As an example, one can consider the scattering of two fermions:

$$
p+k \longrightarrow p^{\prime}+k^{\prime}
$$

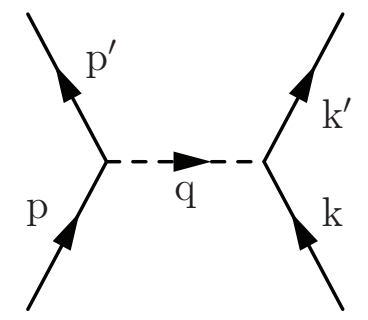

Then the matrix element using the above Feynman rules gives:

$$
\begin{aligned}
i \mathcal{M} & =\bar{u}\left(p^{\prime}\right)(-i \lambda) u(p) \frac{i}{q^{2}-m_{\phi}^{2}} \bar{u}\left(k^{\prime}\right) u(k)(-i \lambda) \\
& =\frac{-i \lambda^{2}}{q^{2}-m_{\phi}^{2}} \bar{u}\left(p^{\prime}\right) u(p) \bar{u}\left(k^{\prime}\right) u(k)
\end{aligned}
$$

where $u$ and $v$ are four component spinors(see [7]) 


\section{B.6 Decay Rate}

The decay rate formula can be obtained from an equation similar to B.33 with the following modifications:

- There is no relative velocity

- $E_{A}=m_{A}$, since the particle is at rest

Consequently, the decay rate is:

$$
d \Gamma=\frac{1}{2 m_{A}}\left(\prod_{f} \int \frac{d^{3} p_{f}}{(2 \pi)^{3} 2 E_{f}}\right) \times\left|\mathcal{M}\left(p_{A} \longrightarrow p_{f}\right)\right|^{2}(2 \pi)^{4} \delta^{(4)}\left(p_{A}-\sum p_{f}\right)
$$

The integral over final states can be written as:

$$
\int d \Pi_{n} \equiv\left(\prod_{f} \int \frac{d^{3} p_{f}}{(2 \pi)^{3} 2 E_{f}}\right)(2 \pi)^{4} \delta^{4}\left(P-\sum P_{f}\right)
$$

The quantity $\int d \Pi_{n}$ is known as the relativistically invariant $n$-body phase space. So evaluating that for a 2-body, one get:

$$
\begin{aligned}
\int d \Pi_{2} & =\int \frac{d^{3} p_{1} d^{3} p_{2}}{(2 \pi)^{3}(2 \pi)^{3} 2 E_{1} 2 E_{2}}(2 \pi)^{4} \delta^{4}\left(P_{T}-P_{1}-P_{2}\right) \\
& =\int \frac{d^{3} p_{1} d^{3} p_{2}}{16 \pi^{2} E_{1} E_{2}} \delta^{3}\left(\overrightarrow{p_{1}}+\overrightarrow{p_{2}}\right) \delta\left(E_{T}-E_{1}-E_{2}\right), \text { doing } \overrightarrow{p_{2}} \text { integration sends } \overrightarrow{p_{2}}=-\overrightarrow{p_{1}} \\
& =\int \frac{d^{3} p_{1}}{16 \pi^{2} E_{1} E_{2}} \delta\left(m-E_{1}-E_{2}\right)
\end{aligned}
$$

Here, the four-vectors are given as (working in the center of mass frame):

$$
P_{T}=(m, \overrightarrow{0}), \quad P_{1}=\left(E_{1}, \overrightarrow{p_{1}}\right), \quad P_{2}=\left(E_{2},-\overrightarrow{p_{1}}\right)
$$

Now $E_{T}=m, E_{1}=\sqrt{p^{2}+m_{1}^{2}}, E_{2}=\sqrt{p^{2}+m_{2}^{2}}$ because $\left\|\overrightarrow{p_{1}}\right\|=\left\|\overrightarrow{p_{2}}\right\|=p$ 
Also rewriting $d^{3} p=p^{2} d p d \Omega$ (differential volume element in momentum space in spherical coordinates), then one arrives at:

$$
\begin{aligned}
\int d \Pi_{2} & =\int \frac{p^{2} d p d \Omega}{16 \pi^{2} E_{1} E_{2}} \delta\left(m-E_{1}-E_{2}\right) \\
& =\int \frac{p^{2} d \Omega}{16 \pi^{2} E_{1} E_{2}} \frac{E_{1} E_{2}}{\|\vec{p}\|\left(E_{1}+E_{2}\right)}, \quad \text { since } \frac{d\left(E_{1}+E_{2}\right)}{d p}=\frac{\|\vec{p}\|}{E_{1}}+\frac{\|\vec{p}\|}{E_{2}}=\frac{E_{1} E_{2}}{\|\vec{p}\|\left(E_{1}+E_{2}\right)} \\
& =\int \frac{\|\vec{p}\| d \Omega}{16 \pi^{2}\left(E_{1}+E_{2}\right)}=\int \frac{\|\vec{p}\| d \Omega}{16 \pi^{2} m}, \quad \text { using } m=E_{c m}=E_{1}+E_{2}
\end{aligned}
$$

\section{B.7 Scalar $\eta$ Width}

The Feynman diagram for the 2-body decay of the scalar $\eta$ is:

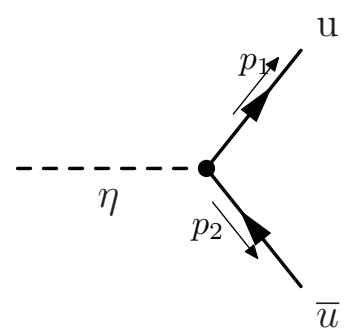

The decay width is:

$$
\begin{aligned}
& \Gamma=\frac{|\mathcal{M}|^{2}}{2 m_{\eta}} \int \mathrm{d} \Pi_{n} \quad \text { Here } \mathrm{n}=2 \text { (number of particles in the final state) } \\
& \text { where } \int \mathrm{d} \Pi_{n} \equiv\left(\prod_{f} \int \frac{\mathrm{d}^{3} p_{f}}{(2 \pi)^{3} 2 E_{f}}\right)(2 \pi)^{4} \delta^{4}\left(P-\sum P_{f}\right)
\end{aligned}
$$

The quantity $\int d \Pi_{n}$ is known as the relativistically invariant n-body phase space. Applying the above expression to our model, the width for $\eta$ becomes:

$$
\Gamma_{\eta}=\frac{1}{2 m_{\eta}} \int \mathrm{d} \Pi_{2}\left|\mathcal{M}\left(m_{\eta} \longrightarrow\left\{P_{1}, P_{2}\right\}\right)\right|^{2}
$$


In general for an unpolarized case $^{1}$, the matrix element is independent of $\theta$ and $\phi$ so the the integral over the solid angle gives $4 \pi$ since the particles in the final state are distinguishable. Furthermore, the matrix element is given by:

$$
i \mathcal{M}=i \epsilon \bar{u}\left(p_{1}\right) v\left(p_{2}\right)
$$

Averaging over the initial and summing over the final spins:

$$
\begin{aligned}
\sum|\mathcal{M}|^{2} & =\sum\left[-i \epsilon \bar{v}\left(p_{2}\right) u\left(p_{1}\right)\right]\left[i \epsilon \bar{u}\left(p_{1}\right) v\left(p_{2}\right)\right] \\
& =\epsilon^{2} \sum \bar{v}\left(p_{2}\right) u\left(p_{1}\right) \bar{u}\left(p_{1}\right) v\left(p_{2}\right) \\
& =\epsilon^{2} \operatorname{tr}\left[\left(\not p_{2}-m_{u}\right)\left(\not p_{1}+m_{u}\right)\right]=\epsilon^{2} \operatorname{tr}\left[\not p_{2} \not p_{1}+m_{u}\left(\not p_{2}-\not p_{1}\right)-m_{u}^{2}\right] \\
& =\epsilon^{2}\left(\operatorname{tr}\left[\not p_{2} \not \not_{1}\right]-\operatorname{tr}\left[m_{u}^{2}\right]\right)=4 \epsilon^{2}\left(p_{1} \cdot p_{2}-m_{u}^{2}\right)
\end{aligned}
$$

Now doing some basic kinematics:

$$
\begin{aligned}
& s=m_{\eta}^{2}=\left(p_{1}+p_{2}\right)^{2}=p_{1}^{2}+p_{2}^{2}+2 p_{1} \cdot p_{2}, \quad \text { using } p_{1}^{2}=p_{2}^{2}=m_{u}^{2} \\
& \Longrightarrow p_{1} \cdot p_{2}=\frac{1}{2} m_{\eta}^{2}-m_{u}^{2} \\
& m_{\eta}=E_{1}+E_{2}=2 E_{1}=2 \sqrt{p_{1}^{2}+m_{u}^{2}} \Longrightarrow\left\|\overrightarrow{p_{1}}\right\|=\frac{m_{\eta}}{2} \sqrt{1-\frac{4 m_{u}^{2}}{m_{\eta}^{2}}}
\end{aligned}
$$

Note that in the width calculation, the colour of the quark has not been taken into account, so to obtain the correct formula one needs to put in the colour factor $N_{c}=3$. Also using the expression $\int d \Pi_{2}=\int \frac{\|\vec{p}\| d \Omega}{16 \pi^{2} m}$ (shown in appendix equation B.40), the

\footnotetext{
${ }^{1}$ Here we have a scalar so it doesn't matter.
} 
width is given by:

$$
\begin{aligned}
\Gamma_{\eta} & =3 \frac{1}{2 m_{\eta}} \frac{\|\vec{p}\|}{16 \pi^{2} m_{\eta}}(4 \pi) 4 \epsilon^{2}\left(p_{1} \cdot p_{2}-m_{u}^{2}\right) \\
& =\frac{3 \epsilon^{2}}{2 \pi m_{\eta}^{2}}\left(\frac{m_{\eta}}{2} \sqrt{1-\frac{4 m_{u}^{2}}{m_{\eta}^{2}}}\right)\left(\frac{1}{2} m_{\eta}^{2}-m_{u}^{2}-m_{u}^{2}\right) \\
& =\frac{3 \epsilon^{2} m_{\eta}}{8 \pi}\left(1-\frac{4 m_{u}^{2}}{m_{\eta}^{2}}\right)^{\frac{3}{2}}
\end{aligned}
$$

\section{B.8 Noether's Theorem}

Noether's Theorem is concerned with continuous symmetries. It basically states that a continuous transformation of a field that leaves the equation of motion invariant leads to conserved quantities corresponding to the given transformation. Now the equation of motion (equation 2.20) is invariant if the Lagrangian is invariant under a transformation of the field. For example, lets suppose that the Lagrangian is invariant under the following transformation

$$
\phi \longrightarrow \phi^{\prime}=\phi+\delta \phi
$$

Then from the invariance we have,

$$
\mathcal{L}(\phi+\delta \phi)=\mathcal{L}(\phi) \Longrightarrow \mathcal{L}(\phi+\delta \phi)-\mathcal{L}(\phi)=0
$$

Applying a Taylor expansion up to leading order in the infinitesimal field transformation,

$$
\frac{\partial \mathcal{L}}{\partial \phi} \delta \phi+\frac{\partial \mathcal{L}}{\partial\left(\partial_{\mu} \phi\right)} \delta\left(\partial_{\mu} \phi\right)=0
$$


Using equation 2.17 and equation 2.20 , we obtain

$$
\begin{aligned}
& {\left[\partial_{\mu} \frac{\partial \mathcal{L}}{\partial\left(\partial_{\mu} \phi\right)}\right] \delta \phi+\frac{\partial \mathcal{L}}{\partial\left(\partial_{\mu} \phi\right)}\left(\partial_{\mu} \delta \phi\right)=0} \\
& \partial_{\mu}\left[\frac{\partial \mathcal{L}}{\partial\left(\partial_{\mu} \phi\right)} \delta \phi\right]=0 \equiv \partial_{\mu} J^{\mu}
\end{aligned}
$$

Hence,

$$
J^{\mu}=\frac{\partial \mathcal{L}}{\partial\left(\partial_{\mu} \phi\right)} \delta \phi, \quad \text { is conserved }
$$




\section{Appendix $\mathrm{C}$}

\section{Additional Plots}

\section{C.1 Pythia8 and Pythia6}

Some extra plots for the discrepancy between Pythia6 and Pythia8, for the $8 \mathrm{TeV}$ CMS analysis [13].

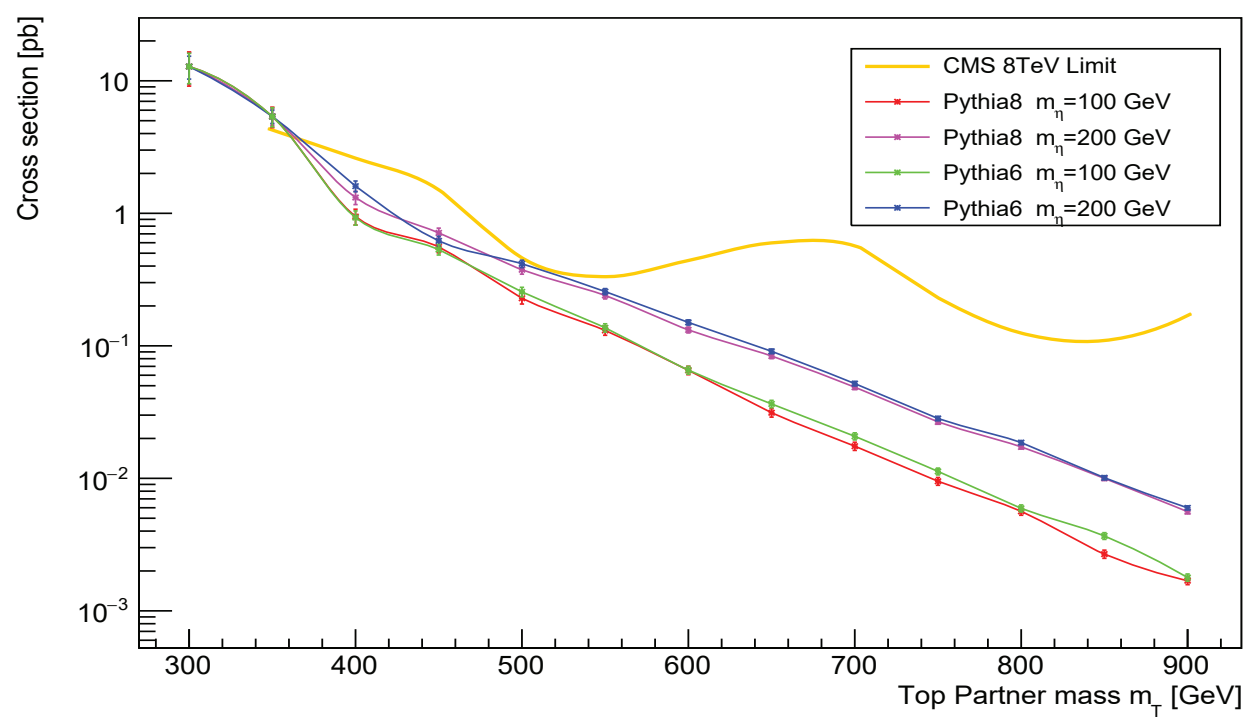

Figure C.1: Pythia8 and Pythia6 cross section curves from CMS 8 TeV analysis for various $\eta$ masses. 
As an illustration, we have plotted the cross section as a function of the top partner mass as shown in figure C.1. The CMS results appear to be very similar to each other for the two versions of Pythia. Unlike the ATLAS search result (figure 4.6), there are very small fluctuations in the cross sections. A plausible explanation for this is due to the fact that the CMS search [13] and the ATLAS search [14] use different event selection requirements. The CMS analysis actually reconstructs the three jet resonances while the ATLAS analysis mainly relies on the transverse momentum of the jets. So some of the events that contribute to the discrepancy are eliminated by the applied cuts in the CMS search but not in the ATLAS search. 


\section{List of References}

[1] G. Bertone, D. Hooper, and J. Silk, "Particle dark matter: Evidence, candidates and constraints," Phys. Rept., vol. 405, pp. 279-390, 2005.

[2] S. P. Martin, "A Supersymmetry primer," 1997. [Adv. Ser. Direct. High Energy Phys.18,1(1998)].

[3] G. Aad et al., "Observation of a new particle in the search for the Standard Model Higgs boson with the ATLAS detector at the LHC," Phys. Lett., vol. B716, pp. 1-29, 2012.

[4] S. Chatrchyan et al., "Observation of a new boson at a mass of $125 \mathrm{GeV}$ with the CMS experiment at the LHC," Phys. Lett., vol. B716, pp. 30-61, 2012.

[5] "Search for pair production of vector-like top partners in events with exactly one lepton and large missing transverse momentum in $\sqrt{s}=13 \mathrm{TeV} p p$ collisions with the ATLAS detector," Tech. Rep. ATLAS-CONF-2016-101, CERN, Geneva, Sep 2016 .

[6] V. Khachatryan et al., "Search for vector-like charge $2 / 3 \mathrm{~T}$ quarks in protonproton collisions at sqrt(s) $=8 \mathrm{TeV}$," Phys. Rev., vol. D93, no. 1, p. 012003, 2016.

[7] D. V. S. Michael E. Peskin, An Introduction to Quantum Field Theory. New York: Westview Press, 1995.

[8] S. Weinberg, "A Model of Leptons," Phys. Rev. Lett., vol. 19, pp. 1264-1266, 1967.

[9] M. D. Schwartz, Quantum Field Theory and the Standard Model. Cambridge University Press, 2014. 
[10] J. Alwall, R. Frederix, S. Frixione, V. Hirschi, F. Maltoni, O. Mattelaer, H. S. Shao, T. Stelzer, P. Torrielli, and M. Zaro, "The automated computation of treelevel and next-to-leading order differential cross sections, and their matching to parton shower simulations," JHEP, vol. 07, p. 079, 2014.

[11] C. Patrignani et al., "Review of Particle Physics," Chin. Phys., vol. C40, no. 10, p. 100001, 2016.

[12] V. Khachatryan et al., "Searches for $R$-parity-violating supersymmetry in ppcollisions at $\sqrt{(s)}=8 \mathrm{TeV}$ in final states with 0-4 leptons," Phys. Rev., vol. D94, no. 11, p. 112009, 2016.

[13] S. Chatrchyan et al., "Searches for light- and heavy-flavour three-jet resonances in pp collisions at $\sqrt{s}=8 \mathrm{TeV}, "$ Phys. Lett., vol. B730, pp. 193-214, 2014.

[14] G. Aad et al., "Search for massive supersymmetric particles decaying to many jets using the ATLAS detector in $p p$ collisions at $\sqrt{s}=8 \mathrm{TeV}, "$ Phys. Rev., vol. D91, no. 11, p. 112016, 2015. [Erratum: Phys. Rev.D93,no.3,039901(2016)].

[15] A. D. Martin, W. J. Stirling, R. S. Thorne, and G. Watt, "Parton distributions for the LHC," Eur. Phys. J., vol. C63, pp. 189-285, 2009.

[16] A. Alloul, N. D. Christensen, C. Degrande, C. Duhr, and B. Fuks, "FeynRules 2.0 - A complete toolbox for tree-level phenomenology," Comput. Phys. Commun., vol. 185, pp. 2250-2300, 2014.

[17] T. Sjostrand, S. Mrenna, and P. Z. Skands, "A Brief Introduction to PYTHIA 8.1," Comput. Phys. Commun., vol. 178, pp. 852-867, 2008.

[18] J. de Favereau, C. Delaere, P. Demin, A. Giammanco, V. Lematre, A. Mertens, and M. Selvaggi, "DELPHES 3, A modular framework for fast simulation of a generic collider experiment," JHEP, vol. 02, p. 057, 2014.

[19] G. Aad et al., "Measurement of multi-jet cross sections in proton-proton collisions at a 7 TeV center-of-mass energy," Eur. Phys. J., vol. C71, p. 1763, 2011.

[20] S. Hoeche, F. Krauss, N. Lavesson, L. Lonnblad, M. Mangano, A. Schalicke, and S. Schumann, "Matching parton showers and matrix elements," in HERA and the LHC: A Workshop on the implications of HERA for LHC physics: Proceedings Part A, pp. 288-289, 2005. 
[21] M. Cacciari, G. P. Salam, and G. Soyez, "The Anti-k(t) jet clustering algorithm," JHEP, vol. 04, p. 063, 2008.

[22] T. Sjostrand, S. Mrenna, and P. Z. Skands, "PYTHIA 6.4 Physics and Manual," JHEP, vol. 05, p. 026, 2006.

[23] L. Lonnblad and S. Prestel, "Matching Tree-Level Matrix Elements with Interleaved Showers," JHEP, vol. 03, p. 019, 2012.

[24] S. Chatrchyan et al., "Search for three-jet resonances in $p p$ collisions at $\sqrt{s}=7$ TeV," Phys. Lett., vol. B718, pp. 329-347, 2012.

[25] D. Krohn, J. Thaler, and L.-T. Wang, "Jet Trimming," JHEP, vol. 02, p. 084, 2010

[26] M. Cacciari, G. P. Salam, and G. Soyez, "FastJet User Manual," Eur. Phys. J., vol. C72, p. 1896, 2012.

[27] T. A. collaboration, "Search for massive supersymmetric particles in multi-jet final states produced in pp collisions at $\sqrt{s}=13 \mathrm{TeV}$ using the ATLAS detector at the LHC," 2016.

[28] T. Junk, "Confidence level computation for combining searches with small statistics," Nucl. Instrum. Meth., vol. A434, pp. 435-443, 1999.

[29] T. Aaltonen et al., "First Search for Multijet Resonances in $\sqrt{s}=1.96 \mathrm{TeV} p \bar{p}$ Collisions," Phys. Rev. Lett., vol. 107, p. 042001, 2011.

[30] D. J. Griffiths, Introduction to elementary particles; 2nd rev. version. Physics textbook, New York, NY: Wiley, 2008.

[31] A. L. Read, "Presentation of search results: the cl s technique," Journal of Physics G: Nuclear and Particle Physics, vol. 28, no. 10, p. 2693, 2002. 